\title{
Matrix supergroup Chern-Simons models for vortex-antivortex systems
}

\author{
Tadashi Okazaki $^{a}$ and Douglas J. Smith ${ }^{b}$ \\ ${ }^{a}$ Department of Physics and Center for Theoretical Sciences, National Taiwan University, \\ Taipei 10617, Taiwan \\ ${ }^{b}$ Department of Mathematical Sciences, Durham University, \\ Lower Mountjoy, Stockton Road, Durham DH1 3LE, U.K. \\ E-mail: tadashiokazaki@phys.ntu.edu.tw, Douglas.Smith@durham.ac.uk
}

Abstract: We study a $\mathrm{U}(N \mid M)$ supermatrix Chern-Simons model with an $\mathrm{SU}(p \mid q)$ internal symmetry. We propose that the model describes a system consisting of $N$ vortices and $M$ antivortices involving $\mathrm{SU}(p \mid q)$ internal spin degrees of freedom. We present both classical and quantum ground state solutions, and demonstrate the relation to Calogero models. We present evidence that a large $N$ limit describes $\mathrm{SU}(p \mid q)$ WZW models. In particular, we derive $\widehat{\mathfrak{s u}}(p \mid q)$ Kac-Moody algebras. We also present some results on the calculation of the partition function involving a supersymmetric generalization of the Hall-Littlewood polynomials, indicating the mock modular properties.

Keywords: Chern-Simons Theories, Field Theories in Lower Dimensions, Matrix Models, Solitons Monopoles and Instantons

ARXIV EPRINT: 1712.01370 


\section{Contents}

1 Introduction $\quad 2$

2 Supermatrix Chern-Simons model 3

2.1 Model 3

$\begin{array}{lll}2.2 & \text { Vortex-antivortex system in multilayers } & 6\end{array}$

2.2.1 Chern-Simons vortex quantum mechanics 6

$\begin{array}{lll}2.2 .2 & \text { Vortices and antivortices in multilayers } & 9\end{array}$

3 Classical solutions $\quad 11$

3.1 Generalized Calogero models 12

$\begin{array}{lll}3.2 & \text { Classical ground state } & 14\end{array}$

4 Quantum states $\quad 24$

4.1 Quantization 24

$\begin{array}{lll}4.2 & \text { Quantum ground states } & 27\end{array}$

$\begin{array}{lll}\text { 4.2.1 Determinant states } & 27\end{array}$

$\begin{array}{lll}\text { 4.2.2 Superdeterminant states — case } 1 & 29\end{array}$

$\begin{array}{ll}\text { 4.2.3 Analysis for } \mathrm{U}(N) & 30\end{array}$

4.2.4 Superdeterminant states — case $2 \quad 32$

$5 \quad$ Kac-Moody algebra 33

5.1 Affine Lie algebra 34

$\begin{array}{lll}5.2 & \text { Affine Lie superalgebra } & 35\end{array}$

$\begin{array}{lll}5.3 & \text { Generalization to } \mathrm{U}(N \mid M) & 38\end{array}$

6 Partition function $\quad 39$

$\begin{array}{lll}6.1 & \text { Definition } & 39\end{array}$

6.2 Computation for $\mathrm{U}(N) \quad 42$

6.3 Comments on general case 44

$\begin{array}{lll}7 & \text { Discussion } & 46\end{array}$

$\begin{array}{ll}\text { A More classical ground states } & 48\end{array}$ 


\section{Introduction}

Matrix Chern-Simons models $[1,2]$ are gauged quantum mechanics models whose Lagrangian is first order in time derivatives. They have remarkable connections to a wide range of topics in physics and mathematics.

Susskind [3] proposed that the infinite-dimensional matrix Chern-Simons quantum mechanical model, as the non-commutative Chern-Simons theory on a plane, could describe the Laughlin theory [4] in such a way that the positions of an infinite number of electrons moving in a two-dimensional plane influenced under strong magnetic field correspond to infinite matrices. Polychronakos [1] subsequently proposed that the finitedimensional $\mathrm{U}(N)$ matrix Chern-Simons model, as the regularized version of the Susskind model, could describe the fractional quantum Hall effect for $N$ electrons. In fact, the matrix Chern-Simons theories share many features with the Laughlin theory in the lowest Landau level [1, 2, 5-11]. The classical ground state of the theory describes an incompressible homogeneous state at the Laughlin filling fractions. Level quantization leads to the specific values of the filling fraction of the Laughlin states [1]. There exists a formal mapping between the quantum physical states of the matrix model and the Laughlin states [5]. Further extension has been studied in $[2,10]$ by introducing an $\mathrm{SU}(p)$ global symmetry in the Polychronakos model. It has been argued that this extended model describes the non-Abelian quantum Hall effect with internal spin degrees of freedom.

Another remarkable application of the $\mathrm{U}(N)$ matrix Chern-Simons model has been proposed by Tong [12]. From the brane construction in type IIB string theory [13] and Manton's analysis [14] of vortices in non-relativistic Chern-Simons theory, he conjectured that the $\mathrm{U}(N)$ matrix Chern-Simons model can be viewed as a description of the lowenergy dynamics of $N$ vortices in non-relativistic Abelian Chern-Simons matter theories. In addition, a further generalization has been argued in [2] that the $\mathrm{U}(N)$ matrix ChernSimons model with an $\mathrm{SU}(p)$ global symmetry is the effective description of $N$ vortices in non-relativistic $\mathrm{U}(p)$ Chern-Simons matter theories.

As shown in [1], the $\mathrm{U}(N)$ matrix Chern-Simons model is equivalent to the Calogero model, which is an integrable system of $N$ non-relativistic particles with pairwise inversesquare interaction. This relation can be achieved by identifying the eigenvalues of the matrix with the coordinates of the particles on a line. Using the relation to the Calogero model, the spectrum of the $\mathrm{U}(N)$ matrix Chern-Simons model has been examined [7].

An exciting link between the $\mathrm{U}(N)$ matrix Chern-Simons model with $\mathrm{SU}(p)$ global symmetry and the $\mathrm{SU}(p)$ Wess-Zumino-Witten (WZW) model has been established in [10]. In the large $N$ limit, the current operators constructed from the matrix degrees of freedom realize the affine Lie algebra $\widehat{\mathfrak{s u}}(p)$, and the partition function of the matrix model turns out to be proportional to the character of the affine Lie algebra. This reflects rather rich mathematical structures of the matrix Chern-Simons model.

We will study a new type of generalization of the matrix Chern-Simons theory, that is a $\mathrm{U}(N \mid M)$ supermatrix Chern-Simons quantum mechanics with an $\mathrm{SU}(p \mid q)$ global symmetry. Mostly we will consider the case where $N \geq M$ and $p \geq q$. 
In section 2 we introduce the model and argue that the model describes the dynamics of $N$ vortices and $M$ antivortices in the non-relativistic Chern-Simons matter theory, motivated by Tong's proposal [12]. The supermatrix field $\widehat{Z}_{A B}$ consists of an $N \times N$ bosonic matrix field $Z_{a b}$, an $M \times M$ bosonic matrix field $\widetilde{Z}_{\alpha \beta}$, an $N \times M$ fermionic matrix field $A_{a \alpha}$ and an $M \times N$ fermionic matrix field $B_{\alpha a}$. While the bosonic fields $Z_{a b}$ and $\widetilde{Z}_{\alpha \beta}$ correspond to the positions on a plane of $N$ vortices and $M$ antivortices respectively, the fermionic $A_{a \alpha}$ and $B_{\alpha a}$ describe interactions between a vortex and an antivortex. In addition, the supervector field $\widehat{\Phi}_{A I}$ describes their internal spin degrees of freedom of the two-dimensional system. In a purely theoretical setup we argue that it can be realized by two types of multilayered structure characterized by strong magnetic fields in opposite directions.

In section 3 we study the classical ground states as lowest energy solutions to classical equations of motion. The model turns out to be related to the generalized Calogero model with $\mathrm{SU}(p \mid q)$ spin degrees of freedom. We find two types of classical ground states. Both configurations admit non-trivial configuration for $Z_{a b}$ which forms a circular droplet of $(N-M)$ vortices as in [1], however, they are distinguished by the positive or negative contributions of vortex-antivortex pairs to the energy. In fact, these are similar to the two types of energy contributions of vortex-antivortex pairs due to different polarizations of the pairs of vortices and antivortices.

In section 4 we study the quantization of the theory. We represent the quantum ground state in terms of a superdeterminant operator.

In section 5 we examine a connection to the WZW model. Following the idea of [10], we construct the current operators from matrix degrees of freedom and demonstrate that this provides the left-moving $\widehat{\mathfrak{s u}}(p \mid q)$ affine Lie superalgebra.

In section 6 we study the spectrum by studying the partition function. We present a general integral expression of the partition function. We argue that it admits an expression in terms of a supersymmetric generalization of the Hall-Littlewood polynomials, indicating potential mock modularity. In particular, for ordinary gauge group we obtain an explicit expression of the partition function in terms of Kostka polynomials and supersymmetric Schur polynomials. From the resulting partition function we show that the ground state energy in section 4 can be correctly reproduced.

In section 7 we conclude and discuss future directions.

\section{Supermatrix Chern-Simons model}

\subsection{Model}

We consider a $\mathrm{U}(N \mid M)$ supermatrix Chern-Simons model whose action is given by

$$
S=\int d t\left[i \operatorname{Str}\left(\widehat{Z}^{\dagger} D_{t} \widehat{Z}\right)+i \sum_{I, A}(-1)^{I} \widehat{\Phi}_{I A}^{\dagger} D_{t} \widehat{\Phi}_{A I}-\kappa \operatorname{Str} \widehat{\alpha}-\omega \operatorname{Str} \widehat{Z}^{\dagger} \widehat{Z}\right]
$$

where indices $A \in\{1, \cdots, N, N+1, \cdots, N+M\}$ denote $\mathrm{U}(N \mid M)$ gauge symmetry and $I \in$ $\{1, \cdots, p, p+1, \cdots p+q\}$ are $\mathrm{SU}(p \mid q)$ flavor symmetry. Also, $(-1)^{I}=1$ for $I \in\{1, \cdots, p\}$ 
and $(-1)^{I}=-1$ for $I \in\{p+1, \cdots p+q\}$. Here

$$
\widehat{Z}_{A B}=\left(\begin{array}{cc}
Z_{a b} & A_{a \beta} \\
B_{\alpha b} & \widetilde{Z}_{\alpha \beta}
\end{array}\right), \quad \widehat{Z}_{A B}^{\dagger}=\left(\begin{array}{cc}
Z_{b a}^{*} & B_{b \alpha}^{*} \\
A_{\beta a}^{*} & \widetilde{Z}_{\beta \alpha}^{*}
\end{array}\right), \quad \widehat{\alpha}_{A B}=\left(\begin{array}{cc}
\alpha_{a b} & \lambda_{a \beta} \\
\widetilde{\lambda}_{\alpha b} & \widetilde{\alpha}_{\alpha \beta}
\end{array}\right)
$$

are the $(N+M) \times(N+M)$ supermatrices where the indices $a, b=1, \cdots, N$ and $\alpha, \beta=$ $1, \cdots, M$ label the bosonic subgroups $\mathrm{U}(N)$ and $\mathrm{U}(M)$ of the supergauge group $\mathrm{U}(N \mid M)$. The supermatrix $\widehat{Z}_{A B}$ involves a bosonic $\mathrm{U}(N)$ adjoint complex scalar field $Z_{a b}$, a bosonic $\mathrm{U}(M)$ adjoint complex scalar $\widetilde{Z}_{\alpha \beta}$, and fermionic bi-fundamental fields $A_{a \beta}$ and $B_{\alpha b}$. The supermatrix $\widehat{\alpha}$ is the $\mathrm{U}(N \mid M)$ supergroup gauge field. It contains a $\mathrm{U}(N)$ bosonic gauge field $\alpha_{a b}$, a $\mathrm{U}(M)$ bosonic gauge field $\widetilde{\alpha}_{\alpha \beta}$, and fermionic bi-fundamental parts $\lambda_{a \beta}, \widetilde{\lambda}_{\alpha b}$ of the supergroup gauge field. Since the gauge field $\widehat{\alpha}$ is Hermitian, so are $\alpha$ and $\widetilde{\alpha}$, while $\widetilde{\lambda}=\lambda^{\dagger}$. The fields

$$
\widehat{\Phi}_{A I}=\left(\begin{array}{cc}
\phi_{a i} & \psi_{a \lambda} \\
\widetilde{\psi}_{\alpha i} & \widetilde{\phi}_{\alpha \lambda}
\end{array}\right), \quad \widehat{\Phi}_{I A}^{\dagger}=\left(\begin{array}{cc}
\phi_{i a}^{\dagger} & \widetilde{\psi}_{i \alpha}^{\dagger} \\
\psi_{\lambda a}^{\dagger} & \widetilde{\phi}_{\lambda \alpha}^{\dagger}
\end{array}\right)=\left(\begin{array}{cc}
\phi_{a i}^{*} & \widetilde{\psi}_{\alpha i}^{*} \\
\psi_{a \lambda}^{*} & \widetilde{\phi}_{\alpha \lambda}^{*}
\end{array}\right)
$$

are arrays of complex $(N \mid M)$ supervectors and $(p \mid q)$ supervectors where the indices $i=$ $1, \cdots, p$ and $\lambda=1, \cdots, q$ label the $\mathrm{SU}(p)$ and $\mathrm{SU}(q)$ global symmetry subgroups. The full global $\mathrm{SU}(p \mid q)$ transformations are

$$
\widehat{\Phi}_{A I} \rightarrow \widehat{\Phi}_{A J} \widehat{M}_{J I}
$$

where $\widehat{M} \in \mathrm{SU}(p \mid q)$. The covariant derivatives are defined by

$$
D_{t} \widehat{Z}=\dot{\widehat{Z}}-i[\widehat{\alpha}, \widehat{Z}], \quad D_{t} \widehat{\Phi}=\dot{\hat{\Phi}}-i \widehat{\alpha} \widehat{\Phi} .
$$

The gauge transformations are

$$
\begin{aligned}
\widehat{Z}_{A B} & \rightarrow \widehat{U}_{A C} \widehat{Z}_{C D} \widehat{U}_{D B}^{\dagger}, \\
\widehat{\Phi}_{A I} & \rightarrow \widehat{U}_{A B} \widehat{\Phi}_{B I}, \\
\widehat{\alpha}_{A B} & \rightarrow \widehat{U}_{A C} \widehat{\alpha}_{C D} \widehat{U}_{D B}^{\dagger}+i \widehat{U}_{A C} \dot{\hat{U}}_{C B}^{\dagger}
\end{aligned}
$$

where

$$
\widehat{U}_{A B}=\left(\begin{array}{cc}
U_{a b} & V_{a \beta} \\
W_{\alpha b} & \widetilde{U}_{\alpha \beta}
\end{array}\right) \in \mathrm{U}(N \mid M)
$$

and since $\widehat{U}$ is unitary

$$
\widehat{U}^{\dagger}=\widehat{U}^{-1}=\left(\begin{array}{cc}
U^{-1}\left(I-V \widetilde{U}^{-1} W U^{-1}\right)^{-1} & -U^{-1} V \widetilde{U}^{-1}\left(I-W U^{-1} V \widetilde{U}^{-1}\right)^{-1} \\
-\widetilde{U}^{-1} W U^{-1}\left(I-V \widetilde{U}^{-1} W U^{-1}\right)^{-1} & \widetilde{U}^{-1}\left(I-W U^{-1} V \widetilde{U}^{-1}\right)^{-1}
\end{array}\right)
$$

Note that we have chosen the $\mathrm{SU}(p \mid q)$ transformations in (2.4) to act from the right on $\widehat{\Phi}$. This is so that these transformations commute with the $\mathrm{U}(N \mid M)$ gauge transformations (2.7) acting from the left. This is only necessary when $M \neq 0$ and $q \neq 0$ since in this 
general case not all components of $\widehat{M}$ commute with all components of $\widehat{U}$, as some pairs anti-commute.

In terms of the elements of the matrices (2.2) and (2.3), the action (2.1) is expressed as

$$
\begin{aligned}
S= & \int d t\left[i \operatorname { T r } \left(Z^{\dagger} D Z+B^{\dagger} D B-i Z^{\dagger}(\lambda B-A \widetilde{\lambda})-i B^{\dagger}(\widetilde{\lambda} Z-\widetilde{Z} \widetilde{\lambda})\right.\right. \\
& \left.-\widetilde{Z}^{\dagger} D \widetilde{Z}-A^{\dagger} D A+i \widetilde{Z}^{\dagger}(\widetilde{\lambda} A-B \lambda)+i A^{\dagger}(\lambda \widetilde{Z}-Z \lambda)\right) \\
& +i \sum\left(\phi^{\dagger} D \phi-i \phi^{\dagger} \lambda \widetilde{\psi}-\psi^{\dagger} D \psi+i \psi^{\dagger} \lambda \widetilde{\phi}-\widetilde{\phi}^{\dagger} D \widetilde{\phi}+i \widetilde{\phi^{\dagger}} \widetilde{\lambda} \psi+\widetilde{\psi}^{\dagger} D \widetilde{\psi}-i \widetilde{\psi}^{\dagger} \widetilde{\lambda} \phi\right) \\
& \left.-\kappa \operatorname{Tr}(\alpha-\widetilde{\alpha})-\omega \operatorname{Tr}\left(Z^{\dagger} Z+B^{\dagger} B-\widetilde{Z}^{\dagger} \widetilde{Z}-A^{\dagger} A\right)\right]
\end{aligned}
$$

where the covariant derivatives are defined by

$$
\begin{aligned}
D Z & =\dot{Z}-i[\alpha, Z], & D \widetilde{Z} & =\dot{\widetilde{Z}}-i[\widetilde{\alpha}, \widetilde{Z}], \\
D A & =\dot{A}-i \alpha A+i A \widetilde{\alpha}, & D B & =\dot{B}-i \widetilde{\alpha} B+i B \alpha, \\
D \phi & =\dot{\phi}-i \alpha \phi, & D \widetilde{\phi} & =\dot{\widetilde{\phi}}-i \widetilde{\alpha} \widetilde{\phi} \\
D \psi & =\dot{\psi}-i \alpha \psi, & D \widetilde{\psi} & =\dot{\widetilde{\psi}}-i \widetilde{\alpha} \widetilde{\psi} .
\end{aligned}
$$

The gauge transformations (2.6) of the fields $\widehat{Z}_{A B}$ are expressed by

$$
\begin{aligned}
& Z \rightarrow U Z U^{\dagger}+V B U^{\dagger}+U A V^{\dagger}+V \widetilde{Z} V^{\dagger}, \\
& \widetilde{Z} \rightarrow \widetilde{U} \widetilde{Z} \widetilde{U}^{\dagger}+W A \widetilde{U}^{\dagger}+\widetilde{U} B W^{\dagger}+W Z W^{\dagger}, \\
& A \rightarrow U A \widetilde{U}^{\dagger}+U Z W^{\dagger}+V \widetilde{Z} \widetilde{U}^{\dagger}+V B W^{\dagger}, \\
& B \rightarrow \widetilde{U} B U^{\dagger}+W Z U^{\dagger}+\widetilde{U} \widetilde{Z} V^{\dagger}+W A V^{\dagger},
\end{aligned}
$$

those of the fields $\widehat{\Phi}_{A I}$ are

$$
\begin{array}{ll}
\phi \rightarrow U \phi+V \widetilde{\psi}, & \widetilde{\phi} \rightarrow \widetilde{U} \widetilde{\phi}+W \psi, \\
\psi \rightarrow U \psi+V \widetilde{\phi}, & \widetilde{\psi} \rightarrow \widetilde{U} \widetilde{\psi}+W \phi,
\end{array}
$$

and those of the gauge fields $\widehat{\alpha}$ are

$$
\begin{aligned}
& \alpha \rightarrow U \alpha U^{\dagger}+V \widetilde{\lambda} U^{\dagger}+U \lambda V^{\dagger}+V \widetilde{\alpha} V^{\dagger}+i U \dot{U}^{\dagger}+i V \dot{V}^{\dagger}, \\
& \widetilde{\alpha} \rightarrow \widetilde{U} \widetilde{\alpha} \widetilde{U}^{\dagger}+W \lambda \widetilde{U}^{\dagger}+\widetilde{U} \widetilde{\lambda} W^{\dagger}+W \alpha W^{\dagger}+i \widetilde{U} \dot{\tilde{U}}^{\dagger}+i W \dot{W}^{\dagger}, \\
& \lambda \rightarrow U \lambda \widetilde{U}^{\dagger}+U \alpha W^{\dagger}+V \widetilde{\alpha} \widetilde{U}^{\dagger}+V \widetilde{\lambda} W^{\dagger}+i U \dot{W}^{\dagger}+i V \dot{\tilde{U}}{ }^{\dagger}, \\
& \widetilde{\lambda} \rightarrow \widetilde{U} \widetilde{\lambda} U^{\dagger}+W \alpha U^{\dagger}+\widetilde{U} \widetilde{\alpha} V^{\dagger}+W \lambda V^{\dagger}+i W \dot{U}^{\dagger}+i \widetilde{U} \dot{V}^{\dagger} .
\end{aligned}
$$

When $M=0$ and $q=0$, our supermatrix Chern-Simons model (2.1) becomes the ordinary matrix Chern-Simons model in $[1,2,10]$

$$
S=\int d t\left[i \operatorname{Tr}\left(Z^{\dagger} D_{t} Z\right)+i \sum_{i=1}^{p} \phi_{i}^{\dagger} D_{t} \phi_{i}-\kappa \operatorname{Tr} \alpha-\omega \operatorname{Tr} Z^{\dagger} Z\right]
$$


where $Z$ is a complex adjoint scalar and $\phi_{i}, i=1, \cdots, p$ are $p$ fundamental complex scalars. Here the covariant derivatives are

$$
D_{t} Z=\dot{Z}-i[\alpha, Z], \quad D_{t} \phi_{i}=\dot{\phi}_{i}-i \alpha \phi_{i}
$$

and the trace is taken over the $\mathrm{U}(N)$ gauge indices. The gauge symmetry transformations (2.13)-(2.18) reduce to

$$
Z \rightarrow U Z U^{\dagger}, \quad \phi_{i} \rightarrow U \phi_{i}
$$

for $U \in \mathrm{U}(N)$. This ordinary matrix Chern-Simons model (2.23) is considered as an effective theory of the fractional quantum Hall states composed of $N$ electrons in the lowest Landau level $[1,2,5]$. Although the matrix $Z$ is not diagonalized, it describes positions of $N$ electrons on the plane. The vectors $\phi_{i}$ describe the internal spin, which is called pseudospin, degrees of freedom of $N$ electrons [2].

\subsection{Vortex-antivortex system in multilayers}

\subsubsection{Chern-Simons vortex quantum mechanics}

The matrix Chern-Simons theory with a $\mathrm{U}(N)$ gauge symmetry and an $\mathrm{SU}(p)$ flavor symmetry has been proposed as an effective theory of $N$ vortices in non-relativistic $\mathrm{U}(p)$ ChernSimons matter theory. We will review the discussion in $[2,11,12,14-16]$.

Let us consider a Chern-Simons matter theory with gauge group

$$
\mathrm{U}(p)_{k^{\prime}, k}=\frac{\mathrm{U}(1)_{k^{\prime}} \times \mathrm{SU}(p)_{k}}{\mathbb{Z}_{p}}
$$

with the relation

$$
k^{\prime}-k p \in p^{2} \mathbb{Z}
$$

and the following Lagrangian $[2]$

$$
\begin{aligned}
S & =S_{\mathrm{CS}}+S_{\text {matter }} \\
S_{\mathrm{CS}} & =-\int d^{3} x\left[\frac{k^{\prime}}{4 \pi} \epsilon^{\mu \nu \rho} a_{\mu} \partial_{\nu} a_{\rho}+\frac{k}{4 \pi} \operatorname{Tr} \epsilon^{\mu \nu \rho}\left(A_{\mu} \partial_{\nu} A_{\rho}-\frac{2 i}{3} A_{\mu} A_{\nu} A_{\rho}\right)-\mu a_{0}\right], \\
S_{\text {matter }} & =\int d^{3} x\left[i \phi_{i}^{\dagger} D_{0} \phi_{i}-\frac{1}{2 m} D_{\alpha} \phi_{i}^{\dagger} D_{\alpha} \phi_{i}-\frac{\pi}{m}\left\{\frac{1}{k^{\prime}}\left(\phi_{i}^{\dagger} \phi_{i}\right)^{2}+\frac{1}{k}\left(\phi_{i}^{\dagger} t^{\alpha} \phi_{i}\right)^{2}\right\}\right]
\end{aligned}
$$

where $\mu, \cdots=0,1,2$ are space-time indices, $\alpha, \cdots=1,2$ are spatial indices. Here $a_{\mu}$ is the $\mathrm{U}(1)$ gauge field, $A_{\mu}$ is the $\mathrm{SU}(p)$ gauge field and $\phi_{i}, i=1, \cdots, p$ are the $p$ fundamental complex bosonic fields. Note that the matter is non-relativistic, having first order time derivatives and obeying Schrödinger-like equations of motion. The action has BPS equations which give the vortex equations

$$
\begin{aligned}
f_{12} & =\frac{2 \pi}{k^{\prime}}\left(\left|\phi_{i}\right|^{2}-\mu\right), \quad F_{12}^{\alpha}=\frac{2 \pi}{k} \phi_{i}^{\dagger} t^{\alpha} \phi_{i}, \\
D_{z} \phi_{i} & =0
\end{aligned}
$$


where $f_{12}=\partial_{1} a_{2}-\partial_{2} a_{1}$ and $F_{12}=\partial_{1} A_{2}-\partial_{2} A_{1}-i\left[A_{1}, A_{2}\right]$. The solutions to the vortex equations (2.29) and (2.30) are not unique and the most general solutions with the vortex number $N$ have $2 p N$ parameters [13]. The space of solutions is the vortex moduli space, $\mathcal{M}_{p, N}$, in which the solutions are parametrized by $2 p N$ collective coordinates $X^{a}, a=$ $1, \cdots, 2 p N$ as $\phi_{i}(x ; X)$ and $A_{\alpha}(x ; X)$.

In order to describe the vortex dynamics, it is important to note that the nonrelativistic action (2.28) is first order in time derivatives. This implies that the vortex moduli space $\mathcal{M}_{p, N}$ is not the configuration space but rather the phase space. In the relativistic theory with second order time derivatives the moduli space is the configuration space and the soliton dynamics is addressed by geodesic motion of a slowly moving particle on the moduli space with respect to the metric $g_{a b}(X)[17]$

$$
S=\int d t\left[g_{a b} \dot{X}^{a} \dot{X}^{b}-V(X)\right]
$$

where $V(X)$ is some potential term. Meanwhile, in the non-relativistic theory with first order time derivatives, the moduli space is the phase space. In general, the low-energy effective description of such soliton dynamics is given by [14]

$$
S=\int d t\left[\mathcal{A}_{a}(X) \dot{X}^{a}-V(X)\right]
$$

Here $\mathcal{A}_{a}$ is the connection one-form on the moduli space which obeys [14, 15]

$$
d \mathcal{A}=\Omega
$$

where $\Omega$ is the Kähler form with respect to the metric $g$ on the moduli space. This fact relates the vortices in the Chern-Simons theories and those in the Yang-Mills theories so that the corresponding effective descriptions for both obey a similar relationship.

To extract such a relationship, Tong [12] uses the construction of vortices in YangMills-Higgs theories via the brane configuration in type IIB string theory [13]

$$
\begin{aligned}
& 0123456789 \\
& p \text { D3 ००० ○ } \\
& \text { NS5 } \circ \circ \circ \circ \circ \circ \\
& N \text { D1 ○ }
\end{aligned}
$$

which is depicted in figure 1. The $p$ D3-branes and the NS5-branes provide the $3 \mathrm{~d} \mathcal{N}=$ $4 \mathrm{U}(p)$ Yang-Mills-Higgs theory and $N$ vortices are realized as the $N$ D1-branes. The dynamics of the $N$ D1-branes is given by the $\mathrm{U}(N)$ gauged quantum mechanics, which includes a gauge field $\alpha$, the real adjoint scalar fields $\sigma^{I}, I=3,4,5$ describing the positions of D1-branes in the $x^{3,4,5}$ directions, the complex adjoint scalar fields $Z$ as $N \times N$ complex matrices, describing the positions of D1-branes in the two-dimensional $x^{1}-x^{2}$ plane, and the fundamental complex scalars $\phi$ as $p \times N$ matrices arising from the D1-D3 strings. The 


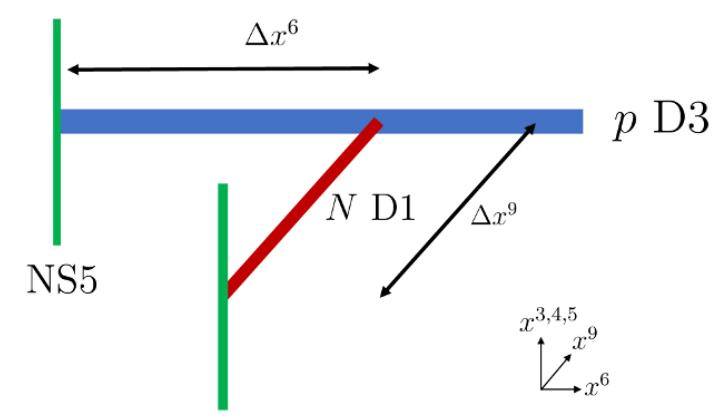

NS5

Figure 1. $N$ Vortices appear as $N$ D1-branes in the $3 \mathrm{~d} \mathcal{N}=4 \mathrm{U}(p)$ Yang-Mills-Higgs theory on $p$ D3-branes attached on NS5-branes.

bosonic part of the Lagrangian is given by

$$
\begin{aligned}
\mathcal{L}= & \operatorname{tr}\left[\frac{1}{2 g^{2}} D_{t} \sigma^{I} D_{t} \sigma^{I}+D_{t} Z^{\dagger} D_{t} Z+D_{t} \phi D_{t} \phi^{\dagger}\right. \\
& \left.-\frac{1}{2 g^{2}}\left[\sigma^{I}, \sigma^{J}\right]^{2}-\left|\left[Z, \sigma^{I}\right]\right|^{2}-\phi \phi^{\dagger} \sigma^{I} \sigma^{I}-\frac{g^{2}}{2}\left(\phi \phi^{\dagger}+\left[Z, Z^{\dagger}\right]-r \mathbb{I}\right)\right]
\end{aligned}
$$

where

$$
D_{t} Z=\dot{Z}-i[\alpha, Z], \quad D_{t} \sigma^{I}=\dot{\sigma}^{I}-i\left[\alpha, \sigma^{I}\right], \quad D_{t} \phi=\dot{\phi}-i \alpha \phi
$$

and the gauge coupling $g$ and the FI parameter $r$ are encoded by the positions of the D3-branes and NS5-branes

$$
\frac{1}{g^{2}}=\frac{2 \pi l_{s}^{2} \Delta x^{9}}{g_{s}}, \quad r=\frac{\Delta x^{6}}{g_{s}} .
$$

The decoupling limit of the D3-brane theory can be achieved in the strong coupling limit $g^{2} \rightarrow \infty$. This leads to the D-term constraints from the leading terms in the Lagrangian (2.35)

$$
\left[Z, Z^{\dagger}\right]+\phi \phi^{\dagger}-r \mathbb{I}=0
$$

According to the $N^{2}$ constraints (2.38), the $\left(N^{2}+p N\right)$ original matrix degrees of freedom from the matrices $Z$ and $\phi$ reduce to $p N$ complex ( $2 p N$ real) degrees of freedom as required from the dimensions of the vortex moduli space $\mathcal{M}_{p, N}$.

From the above analysis via string theory, we see that the dynamics of $N$ vortices in $\mathrm{U}(p)$ Yang-Mills theory is captured by the matrix model (2.35) with the constraints (2.38). To find the matrix model of Chern-Simons vortices, we observe the following facts:

1. The Kähler form $\Omega$ on $\mathcal{M}_{p, N}$ can be constructed from the canonical Kähler form on the space of unconstrained $Z$ and $\phi$ by imposing the non-trivial constraint (2.38) via the symplectic quotient construction.

2. The general action (2.32) is first order in time derivatives. 
It then turns out that the above necessary properties follow from the matrix $\mathrm{U}(N)$ ChernSimons models (2.23) with $\mathrm{SU}(p)$ flavor symmetry in such a way that the auxiliary gauge field $\alpha$ plays a role of a Lagrange multiplier which yields the Gauss law constraints as (2.38).

This fact further instructs us to consider our supermatrix $\mathrm{U}(N \mid M)$ Chern-Simons model (2.1) with an $\mathrm{SU}(p \mid q)$ flavor symmetry as the microscopic description of the system which involves $N$ vortices and $M$ anti-vortices with internal $\mathrm{SU}(p \mid q)$ spin degrees of freedom. We will provide supporting evidence for this interpretation.

\subsubsection{Vortices and antivortices in multilayers}

Vortex-antivortex pairs. A vortex and an antivortex are distinguished by the winding number or vortex number in such a way that a vortex carries the winding number +1 and an antivortex does -1 . When a vortex and an antivortex meet, they can form a vortexantivortex pair. Below a certain temperature, the thermal energy is not enough to generate vortices, however, the lower energy vortex-antivortex pairs can occur. Vortex-antivortex pairs can be localized configurations. The two-dimensional superfluid phase that is characterized by the existence of vortex-antivortex pairs is called the Berezinskii-KosterlitzThouless (BKT) phase [18]. ${ }^{1}$ In addition to winding number, vortices and antivortices are also characterized by polarity $p$ [20]. The polarity is an out-of plane magnetization at the vortex-core which can either point up $(p>0)$ or down $(p<0)$. The winding number $N$ and polarity $p$ specify the circulation or vorticity $q$ by [20]

$$
q=-2 \pi N p
$$

In general the topology and the dynamics of pairs of vortices depend on the circulation. Let $q_{1}$ and $q_{2}$ be circulations of vortices. The kinetic energy of the pairs per unit mass in the plane is given by [21]

$$
E=\pi\left[q_{1}^{2} \ln \frac{R_{0}}{a_{0}}+q_{2}^{2} \ln \frac{R_{0}}{a_{0}}+2 q_{1} q_{2} \ln \frac{R_{0}}{d}\right]
$$

where $R_{0}$ is the size of container, $a_{0}$ is the vortex core radius and $d$ is the separation of pairs. For vortex-antivortex pairs the winding numbers are taken to be opposite and therefore the circulations are determined by their polarities. Dynamics of pairs of vortices have been studied in [21] and vortex-antivortex pairs have been studied numerically in [20, 22, 23]. Let us briefly review the properties of vortex-antivortex pairs.

\section{Parallel polarized vortex-antivortex pairs}

When the vortex and antivortex cores are polarized parallel to each other, they have the opposite circulation according to (2.39). Then the interaction energy in (2.40) is negative binding energy. Qualitatively this is because the flow fields of vortices and antivortices tend to cancel in the bulk and the total kinetic energy is reduced. Consequently the cores of vortices approach each other on spiraling orbits and meet in the center.

\footnotetext{
${ }^{1}$ In the quantum Hall states, a vortex-antivortex can be viewed as a quasiparticle-hole [19].
} 


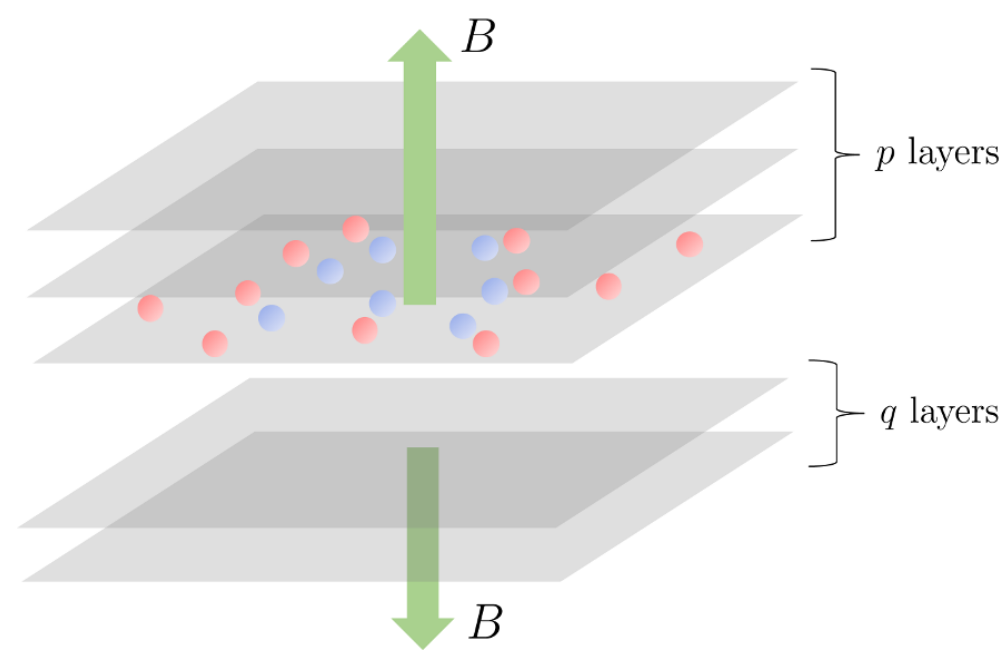

Figure 2. $N$ vortices (red) and $M$ antivortices (blue) in multiple $p$ layers under the influence of vertically upward magnetic field $B$ and $q$ layers under that of the downward magnetic field.

\section{Antiparallel polarized vortex-antivortex pairs}

Because of (2.39), when the vortex and antivortex cores are polarized antiparallel to each other, they have the same circulation. From (2.40) the interaction energy is positive in this case as the flow fields tend to enlarge in the bulk and the total kinetic energy increases. This indicates that after the creation of a vortex-antivortex pair, the antivortex quickly moves towards the original vortex in a rapid process and they then annihilate each other. ${ }^{2}$ It has been shown that such vortex-antivortex annihilation is connected with the emission of sound waves [21, 25-28].

We will see in section 3.2 that the classical ground states in our supermatrix Chern-Simons model (2.1) support these two different types of vortex-antivortex pairs.

Vortices in multilayers. Multilayered quantum Hall systems and vortices have been constructed and studied in theoretical and experimental setup [29-33]. In this case electrons or vortices may occupy several layers and carry different spins in such a way that additional layer indices label them with internal spins. Recently it has been proposed in [2] that the fractional quantum Hall states or vortices in $p$-component systems can be described by the matrix Chern-Simons model (2.23) with an internal $\mathrm{SU}(p)$ symmetry. Here we will consider a generalization of this model with an internal $\mathrm{SU}(p \mid q)$ symmetry. It is expected that our generalized model may be theoretically realized in a system which consists of $N$ vortices and $M$ antivortices in two sets of multilayers as shown in figure 2 where one set of $p$ multilayers is put in a perpendicular magnetic field pointing upward whereas the other $q$ are in the downward magnetic field.

\footnotetext{
${ }^{2}$ See $[24]$ for the detail of the magnetization dynamics of such annihilation process.
} 


\section{Classical solutions}

We first derive the classical equations of motion and Gauss law constraints for the general case. We note that the model is related to generalized Calogero models. We then investigate classical ground states and their physical interpretation, focussing on various special cases.

The classical equations of motion for dynamical scalar fields and fermions from $\widehat{Z}$ read

$$
\begin{aligned}
i(D Z)_{a b} & =A_{a \alpha} \widetilde{\lambda}_{\alpha a}-\lambda_{a \alpha} B_{\alpha b}+\omega Z_{a b}, \\
i(D \widetilde{Z})_{\alpha \beta} & =B_{\alpha a} \lambda_{a \alpha}-\widetilde{\lambda}_{\alpha a} A_{a \beta}+\omega \widetilde{Z}_{\alpha \beta}, \\
i(D A)_{a \alpha} & =Z_{a b} \lambda_{b \alpha}-\lambda_{a \beta} \widetilde{Z}_{\beta \alpha}+\omega A_{a \alpha}, \\
i(D B)_{\alpha a} & =\widetilde{Z}_{\alpha \beta} \widetilde{\lambda}_{\beta a}-\widetilde{\lambda}_{\alpha b} Z_{b a}+\omega B_{\alpha a}
\end{aligned}
$$

and those from $\widehat{\Phi}$ are

$$
\begin{array}{rlrl}
i(D \phi)_{a i} & =-\lambda_{a \alpha} \widetilde{\psi}_{\alpha i}, & i(D \widetilde{\phi})_{\alpha \lambda} & =-\widetilde{\lambda}_{\alpha a} \psi_{a \lambda}, \\
i(D \psi)_{a \lambda} & =-\lambda_{a \alpha} \widetilde{\phi}_{\alpha \lambda}, & i(D \widetilde{\psi})_{\alpha i}=-\widetilde{\lambda}_{\alpha a} \phi_{a i} .
\end{array}
$$

For $M=0$ and $q=0$ the classical equations of motion for the scalar fields are

$$
i D_{t} Z=\omega Z, \quad D_{t} \phi_{i}=0 .
$$

The equations of motion for gauge fields lead to the Gauss law constraints. Using notation $(-1)^{A}=1$ if $A=a$ and $(-1)^{A}=-1$ if $A=\alpha$ etc. the relevant part of the action is

$$
\begin{aligned}
S_{\text {gauge }} & =\int d t\left((-1)^{A} \alpha_{A B}\left[\widehat{Z}, \widehat{Z}^{\dagger}\right]_{B A}+(-1)^{I} \widehat{\Phi}_{I A}^{\dagger} \alpha_{A B} \widehat{\Phi}_{B I}-\kappa(-1)^{A} \alpha_{A A}\right) \\
& =\int d t \alpha_{A B}(-1)^{A}\left(\left[\widehat{Z}, \widehat{Z}^{\dagger}\right]_{B A}+(-1)^{A+I+(A+B)(A+I)} \widehat{\Phi}_{I A}^{\dagger} \widehat{\Phi}_{B I}-\kappa \delta_{A B}\right) \\
& =\int d t \alpha_{A B}(-1)^{A}\left(\left[\widehat{Z}, \widehat{Z}^{\dagger}\right]_{B A}+\widehat{\Phi}_{B I} \widehat{\Phi}_{I A}^{\dagger}-\kappa \delta_{A B}\right) .
\end{aligned}
$$

The Gauss law constraints are therefore

$$
\left[\widehat{Z}, \widehat{Z}^{\dagger}\right]_{A B}+\widehat{\Phi}_{A I} \widehat{\Phi}_{I B}^{\dagger}-\kappa \delta_{A B}=0
$$

or in component form

$$
\begin{aligned}
& {\left[Z, Z^{\dagger}\right]+A A^{\dagger}-B^{\dagger} B+\sum_{i} \phi_{i} \phi_{i}^{\dagger}+\sum_{\lambda} \psi_{\lambda} \psi_{\lambda}^{\dagger}-\kappa \mathbb{I}=0,} \\
& {\left[\widetilde{Z}, \widetilde{Z}^{\dagger}\right]+B B^{\dagger}-A^{\dagger} A+\sum_{\lambda} \widetilde{\phi}_{\lambda} \widetilde{\phi}_{\lambda}^{\dagger}+\sum_{i} \widetilde{\psi}_{i} \widetilde{\psi}_{i}^{\dagger}-\kappa \mathbb{I}=0,} \\
& B Z^{\dagger}-\widetilde{Z}^{\dagger} B+\widetilde{Z} A^{\dagger}-A^{\dagger} Z+\sum_{i} \widetilde{\psi}_{i} \phi_{i}^{\dagger}+\sum_{\lambda} \widetilde{\phi}_{\lambda} \psi_{\lambda}^{\dagger}=0, \\
& Z B^{\dagger}-B^{\dagger} \widetilde{Z}-Z^{\dagger} A+A \widetilde{Z}^{\dagger}+\sum_{i} \phi_{i} \widetilde{\psi}_{i}^{\dagger}+\sum_{\lambda} \psi_{\lambda} \widetilde{\phi}_{\lambda}^{\dagger}=0 .
\end{aligned}
$$

Note that (3.13) follows from the Hermitian conjugation of (3.12). 
Tracing the $\mathrm{U}(N)$ and $\mathrm{U}(M)$ parts of the Gauss law constraints (3.10) and (3.11) give respectively

$$
\begin{aligned}
& \sum_{a} \sum_{\alpha}\left(A_{a \alpha} A_{\alpha a}^{\dagger}+B_{\alpha a} B_{a \alpha}^{\dagger}\right)+\sum_{i} \sum_{\alpha} \phi_{i a}^{\dagger} \phi_{a i}-\sum_{\lambda} \sum_{a} \psi_{\lambda a}^{\dagger} \psi_{a \lambda}=\kappa N, \\
& \sum_{a} \sum_{\alpha}\left(A_{a \alpha} A_{\alpha a}^{\dagger}+B_{\alpha a} B_{a \alpha}^{\dagger}\right)+\sum_{\lambda} \sum_{\alpha} \widetilde{\phi}_{\lambda \alpha}^{\dagger} \widetilde{\phi}_{\alpha \lambda}-\sum_{i} \sum_{\alpha} \widetilde{\psi}_{i \alpha}^{\dagger} \widetilde{\psi}_{\alpha i}=\kappa M
\end{aligned}
$$

and the difference of these equations is the supertrace of the Gauss law constraints

$$
\sum_{i} \sum_{\alpha} \phi_{i a}^{\dagger} \phi_{a i}-\sum_{\lambda} \sum_{a} \psi_{\lambda a}^{\dagger} \psi_{a \lambda}-\sum_{\lambda} \sum_{\alpha} \widetilde{\phi}_{\lambda \alpha}^{\dagger} \widetilde{\phi}_{\alpha \lambda}+\sum_{i} \sum_{\alpha} \widetilde{\psi}_{i \alpha}^{\dagger} \widetilde{\psi}_{\alpha i}=\kappa(N-M) .
$$

Now, we can find explicit solutions after first gauge fixing. The simplest is to choose the temporal gauge $\widehat{\alpha}=0$. Then the equations of motion for the supervector field $\widehat{\Phi},(3.5)$ and (3.6) become $\dot{\hat{\Phi}}=0$. Thus $\widehat{\Phi}$ should be constant. Meanwhile the equations of motion for the supermatrix field $\widehat{Z},(3.1)-(3.4)$ become

$$
\begin{gathered}
\dot{Z}_{a b}=-i \omega Z_{a b}, \\
\dot{\widetilde{Z}}_{\alpha \beta}=-i \omega \widetilde{Z}_{\alpha \beta}, \\
\dot{A}_{a \alpha}=-i \omega A_{a \alpha}, \\
\dot{B}_{\alpha a}=-i \omega B_{\alpha a}
\end{gathered}
$$

and therefore each block matrix has a simple time dependence given by a factor $e^{-i \omega t}$. In the Gauss law constraints (3.10)-(3.13) these time-dependent phases cancel, so in the temporal gauge the classical solutions correspond to time-independent solutions of the Gauss law constraints. Note that we still have the residual gauge symmetry of arbitrary time-independent gauge transformations.

\subsection{Generalized Calogero models}

The Chern-Simons supermatrix quantum mechanics models (2.1) can also be related to generalized Calogero models. To do this, first split $\widehat{Z}$ into its Hermitian and anti-Hermitian parts as

$$
\widehat{Z}=\frac{1}{\sqrt{2}}(\widehat{X}+i \widehat{Y})
$$

where $\widehat{X}$ and $\widehat{Y}$ are both Hermitian. Then the $\mathrm{U}(N \mid M)$ symmetry can be used to diagonalize $\widehat{X}$ which we write as $\widehat{X}_{A B}=x_{A} \delta_{A B}$. In this gauge we find

$$
\left[\widehat{Z}, \widehat{Z}^{\dagger}\right]_{A B}=-i\left(x_{A}-x_{B}\right) Y_{A B}
$$

so the Gauss law constraints (3.9) become

$$
\left(x_{A}-x_{B}\right) \widehat{Y}_{A B}=i \kappa \delta_{A B}-i \widehat{\Phi}_{A I} \widehat{\Phi}_{I B}^{\dagger} .
$$


Clearly the 1.h.s. vanishes for $A=B$ so we see that for all $A$ (but summing over $I$ )

$$
(-1)^{I+A} \widehat{\Phi}_{I A}^{\dagger} \widehat{\Phi}_{A I}=\widehat{\Phi}_{A I} \widehat{\Phi}_{I A}^{\dagger}=\kappa
$$

and there is no constraint on the diagonal elements of $\widehat{Y}$ so we can label $\widehat{Y}_{A A}=y_{A}$.

Note that up to total derivative terms the kinetic term in the Lagrangian is

$$
i \operatorname{Str}\left(\widehat{Z}^{\dagger} \partial_{t} \widehat{Z}\right) \simeq \operatorname{Str}\left(\widehat{Y} \partial_{t} \widehat{X}\right)=\sum_{A}(-1)^{A} y_{A} \dot{x}_{A}
$$

so $(-1)^{A} y_{A}$ is the conjugate momentum to $x_{A}$. Since the coordinates $x_{A}$ are unconstrained, they are generically distinct so we can just divide by $\left(x_{A}-x_{B}\right)$ in (3.23) to find the offdiagonal components of $\widehat{Y}_{A B}$. We can then write

$$
\widehat{Y}_{A B}=y_{A} \delta_{A B}+\frac{i}{x_{A}-x_{B}}\left(\kappa \delta_{A B}-\widehat{\Phi}_{A I} \widehat{\Phi}_{I B}^{\dagger}\right)
$$

with the understanding that the second term vanishes for $A=B$ due to the constraint (3.24).

We can now write the Hamiltonian in terms of the coordinates $x_{A}$ and their conjugate momenta as

$$
\begin{aligned}
& H=\omega \operatorname{Str}\left(\widehat{Z}^{\dagger} \widehat{Z}\right)=\frac{\omega}{2} \operatorname{Str}\left(\widehat{X}^{2}+\widehat{Y}^{2}\right)=\frac{\omega}{2} \sum_{a}\left(x_{a}^{2}+y_{a}^{2}\right)-\frac{\omega}{2} \sum_{\alpha}\left(x_{\alpha}^{2}+y_{\alpha}^{2}\right)+V, \\
& V=\frac{\omega}{2} \sum_{A \neq B} \frac{(-1)^{A}}{\left(x_{A}-x_{B}\right)^{2}} \widehat{\Phi}_{A I} \widehat{\Phi}_{I B}^{\dagger} \widehat{\Phi}_{B J} \widehat{\Phi}_{J A}^{\dagger} .
\end{aligned}
$$

It is possible to interpret this as a model of $N+M$ particles. However, while $N$ have a standard kinetic term, $M$ have the wrong sign for the kinetic term. At the level of equations of motion this is not a problem but it is likely problematic to treat the quantum system. Note, however, that the original system had only first order derivative terms which are well-defined for either sign of kinetic term. In fact, we will see in section 4.1 that it can be quantized to produce a Hamiltonian bounded from below. Clearly, this indicates some subtleties in relating the matrix quantum mechanics to the Calogero model. Nevertheless, it may be possible to interpret the model as a coupling of an $\mathrm{N}$-particle Calogero model to an $M$-particle model with a specific interaction potential. In particular we see that

$$
\begin{aligned}
H & =H_{N}+H_{M}+V_{\text {int }}, \\
H_{N} & =\frac{\omega}{2} \sum_{a}\left(x_{a}^{2}+y_{a}^{2}\right)+\frac{\omega}{2} \sum_{a, b} \frac{1}{\left(x_{a}-x_{b}\right)^{2}} \widehat{\Phi}_{a I} \widehat{\Phi}_{I b}^{\dagger} \widehat{\Phi}_{b J} \widehat{\Phi}_{J a}^{\dagger}, \\
H_{M} & =-\frac{\omega}{2} \sum_{\alpha}\left(x_{\alpha}^{2}+y_{\alpha}^{2}\right)-\frac{\omega}{2} \sum_{\alpha, \beta} \frac{1}{\left(x_{\alpha}-x_{\beta}\right)^{2}} \widehat{\Phi}_{\alpha I} \widehat{\Phi}_{I \beta}^{\dagger} \widehat{\Phi}_{\beta J} \widehat{\Phi}_{J \alpha}^{\dagger}, \\
V_{\text {int }} & =\frac{\omega}{2} \sum_{a, \beta} \frac{1}{\left(x_{a}-x_{\beta}\right)^{2}} \widehat{\Phi}_{a I} \widehat{\Phi}_{I \beta}^{\dagger} \widehat{\Phi}_{\beta J} \widehat{\Phi}_{J a}^{\dagger}-\frac{\omega}{2} \sum_{\alpha, b} \frac{1}{\left(x_{\alpha}-x_{b}\right)^{2}} \widehat{\Phi}_{\alpha I} \widehat{\Phi}_{I b}^{\dagger} \widehat{\Phi}_{b J} \widehat{\Phi}_{J \alpha}^{\dagger} \\
& =\omega \sum_{a, \beta} \frac{1}{\left(x_{a}-x_{\beta}\right)^{2}} \widehat{\Phi}_{a I} \widehat{\Phi}_{I \beta}^{\dagger} \widehat{\Phi}_{\beta J} \widehat{\Phi}_{J a}^{\dagger} .
\end{aligned}
$$


This reduces to the usual Calogero model in the case $M=q=0$ and $p=1$. The constraints (3.24) for the single fundamental scalar $\phi$ give (up to unimportant phases which cancel in the potential)

$$
\phi_{a}=\sqrt{k} \forall a .
$$

We then recognize the usual Calogero model

$$
H=\frac{\omega}{2} \sum_{a}\left(x_{a}^{2}+y_{a}^{2}\right)+\frac{\omega}{2} \sum_{a \neq b} \frac{\kappa^{2}}{\left(x_{a}-x_{b}\right)^{2}} .
$$

If we allow arbitrary $p$, but still $M=q=0$, the potential is generalized to

$$
V=\frac{\omega}{2} \sum_{a \neq b} \frac{\kappa^{2}}{\left(x_{a}-x_{b}\right)^{2}}\left(\phi_{a i} \phi_{i b}^{\dagger}\right)\left(\phi_{b j} \phi_{j a}^{\dagger}\right)
$$

with the constraints

$$
\phi_{a i} \phi_{i a}^{\dagger}=\kappa \forall a
$$

Further generalizing to $q \neq 0$ we get a potential containing terms quadratic and quartic in fermions $\psi_{\lambda a}$ :

$$
V=\frac{\omega}{2} \sum_{a \neq b} \frac{\kappa^{2}}{\left(x_{a}-x_{b}\right)^{2}}\left(\phi_{a i} \phi_{i b}^{\dagger}+\psi_{a \lambda} \psi_{\lambda b}^{\dagger}\right)\left(\phi_{b j} \phi_{j a}^{\dagger}+\psi_{b \rho} \psi_{\rho a}^{\dagger}\right)
$$

with the constraints

$$
\phi_{a i} \phi_{i a}^{\dagger}+\psi_{a \lambda} \psi_{\lambda a}^{\dagger}=\kappa \forall a
$$

Such generalized $N$-particle Calogero models with $\mathrm{SU}(p \mid q)$ internal degrees of freedom, and the related spin chain models - so-called supersymmetric Polychronakos models which arise as their freezing limit, have been studied in [34-37]. We also note that this model can be embedded in specific angular momentum sectors of the ordinary bosonic $\mathrm{U}(N)$ matrix model without any vector-like fields, i.e. with $p=q=0$ or Chern-Simons term. In particular, upon quantization the conserved angular momenta $\left[Z, Z^{\dagger}\right]$ become integer representation $\mathrm{SU}(N)$ generators with vanishing $\mathbb{Z}_{N}$ charge. However, any such representation of $\mathrm{SU}(N)$ can be obtained from a set of bosonic and fermionic oscillators by the Schwinger construction. These oscillators correspond to the fields $\phi_{a i}$ and $\psi_{a \lambda}$ in (3.37) while (3.38) imposes the integrality condition. ${ }^{3}$

\subsection{Classical ground state}

The classical ground state is the classical solution of least energy, so we need to find the time-independent solution to the Gauss law constraints which minimizes the Hamiltonian

$$
H=\omega \operatorname{Str}\left(\widehat{Z}^{\dagger} \widehat{Z}\right)
$$

\footnotetext{
${ }^{3}$ We thank A. Polychronakos for pointing this out.
} 
The Gauss law constraint can be imposed using a Lagrange multiplier. As such a timeindependent Lagrange multiplier appears in exactly the same way as the original gauge field, we use the same notation $\widehat{\alpha}$. So, we must minimize

$$
H=\operatorname{Str}\left(\omega \widehat{Z}^{\dagger} \widehat{Z}-\widehat{\alpha}\left(\left[\widehat{Z}, \widehat{Z}^{\dagger}\right]+\widehat{\Phi}_{I} \widehat{\Phi}_{I}^{\dagger}-\kappa I\right)\right) .
$$

Varying with respect to $\widehat{Z}^{\dagger}$ we see that the result is that the ground state is given by a solution to the Gauss law constraint where also for some $\widehat{\alpha}$

$$
\omega \widehat{Z}=[\widehat{\alpha}, \widehat{Z}] .
$$

As finding the general solution is not simple, let us now consider explicitly the case where $p=1$ and $q=1$. Up to gauge transformations, we take the following generic configuration

$$
\phi_{a}=\left(\begin{array}{c}
0 \\
\vdots \\
0 \\
x
\end{array}\right), \quad \widetilde{\psi}_{\alpha}=\left(\begin{array}{c}
0 \\
\vdots \\
0 \\
0
\end{array}\right), \quad \widetilde{\phi}_{\alpha}=\left(\begin{array}{c}
0 \\
\vdots \\
0 \\
z
\end{array}\right), \quad \psi_{a}=\left(\begin{array}{c}
0 \\
\vdots \\
0 \\
y
\end{array}\right) .
$$

Then the Gauss law constraints become

$$
\begin{aligned}
{\left[\widehat{Z}, \widehat{Z}^{\dagger}\right]_{A B}=} & \left(\kappa-\left(x^{\dagger} x-y^{\dagger} y\right) \delta_{A N}-z^{\dagger} z \delta_{A(N+M)}\right) \delta_{A B}-y z^{\dagger} \delta_{A N} \delta_{B(N+M)}-z y^{\dagger} \delta_{A(N+M)} \delta_{B M} \\
= & \left(\kappa-z^{\dagger} z\left(\delta_{A N}+\delta_{A(N+M)}\right)-\kappa(N-M) \delta_{A N}\right) \delta_{A B}-y z^{\dagger} \delta_{A N} \delta_{B(N+M)} \\
& -z y^{\dagger} \delta_{A(N+M)} \delta_{B N}
\end{aligned}
$$

where the second line takes into account the constraint

$$
x^{\dagger} x-y^{\dagger} y-z^{\dagger} z=\kappa(N-M)
$$

on $x, y$ and $z$ imposed by (3.16), the supertrace of the Gauss law constraints (3.9).

We can also use the residual $\mathrm{U}(N-1 \mid M-1)$ symmetry to partially diagonalize $\widehat{\alpha}$ :

$$
\begin{array}{rlrl}
\alpha_{a b} & =\beta_{a} \delta_{a b}, & a, b \neq N, \\
\widetilde{\alpha}_{\alpha \beta}=\widetilde{\beta}_{\alpha} \delta_{\alpha \beta}, & \alpha, \beta \neq M .
\end{array}
$$

For generic $\beta_{a}$ and $\widetilde{\beta}_{\alpha}$ this reduces the symmetry $\mathrm{U}(N-1 \mid M-1)$ to its maximal Abelian subgroup, i.e. the Cartan subgroup so that the diagonal components correspond to the generators of the Cartan subalgebra. Of course, the residual symmetry will be enhanced to a non-Abelian subgroup of $\mathrm{U}(N \mid M)$ if there is any degeneracy in the values of $\beta_{a}$ and $\widetilde{\beta}_{\alpha}$.

Finally, varying with respect to $\widehat{\Phi}_{I}^{\dagger}$ gives

$$
\widehat{\alpha} \widehat{\Phi}_{I}=0
$$

which for the above configuration for $\widehat{\Phi}$ imposes additional constraints on $\widehat{\alpha}$ and $\widehat{\Phi}$ which can be described in terms of three cases: 
1. $\widehat{\alpha}_{A N}=\widehat{\alpha}_{A(M+N)}=0$ so that $\widehat{\alpha}$ is completely diagonal ${ }^{4}$ but there are no further restrictions on $\widehat{\Phi}$.

2. $x=y=\widehat{\alpha}_{A(M+N)}=0$.

3. $z=\widehat{\alpha}_{A N}=0$.

We comment on the first case in appendix A. In case 2 both $\phi$ and $\psi$ vanish so this is equivalent to considering solutions in the case $p=0$ and $q=1$. We now consider the third case, but as we will see, we can find solutions taking the more restrictive ansatz

$$
\alpha_{N N}=0, \quad z=\widetilde{\phi}_{M}=0 .
$$

Then the equations for $\widehat{Z}_{A B}$, (3.41) reduce to

$$
\begin{aligned}
\left(\beta_{a}-\beta_{b}-\omega\right) Z_{a b} & =0, & & \left(\widetilde{\beta}_{\alpha}-\widetilde{\beta}_{\beta}-\omega\right) \widetilde{Z}_{\alpha \beta}=0, \\
\left(\beta_{a}-\widetilde{\beta}_{\alpha}-\omega\right) A_{a \alpha} & =0, & & \left(\widetilde{\beta}_{\alpha}-\beta_{a}-\omega\right) B_{\alpha a}=0 .
\end{aligned}
$$

It follows that the diagonal parts of $Z$ and $\widetilde{Z}$ are zero. Let $\boldsymbol{\alpha}^{a}$ be the diagonal matrices associated to the simple roots which form a complete set of diagonal matrices

$$
\boldsymbol{\alpha}^{a}=\boldsymbol{\alpha}_{i}^{a} H_{i}=\frac{1}{2} \operatorname{diag}(0,0, \cdots, 0, \underbrace{1}_{a}, \underbrace{-1}_{a+1}, 0 \cdots, 0)
$$

where

$$
H_{m}=\operatorname{diag}(\underbrace{1,1, \cdots, 1}_{m},-m, 0, \cdots, 0)
$$

is the generator of the Cartan subalgebra and the diagonal parts $\alpha_{D}$ of the gauge fields $\alpha$ can be written as

$$
\alpha_{D}=\sum_{a=1}^{N-1} c_{a} \boldsymbol{\alpha}^{a}
$$

It follows that

$$
\operatorname{Tr}\left(\alpha_{D} \cdot \boldsymbol{\alpha}^{a}\right)=\frac{1}{2} c_{a}=\beta_{a}-\beta_{a+1}
$$

where

$$
\alpha_{D}=\operatorname{diag}\left(\beta_{1}, \beta_{2}, \cdots, \beta_{N}\right) .
$$

Thus $\left\{\beta_{a}-\beta_{b}=\omega\right\}$ corresponds to simple roots $\left\{\epsilon_{a}-\epsilon_{b}\right\}$ of $A_{N-1}$ so that the associated canonical variables $Z_{a b}$ take values in $\mathbb{C} E_{a b}$ where $\left\{\epsilon_{1}, \cdots, \epsilon_{N}\right\}$ is the basis of the root

\footnotetext{
${ }^{4}$ However, note that this solution with $\alpha_{N N}=\widetilde{\alpha}_{M M}=0$ involving the same values for $\widehat{\alpha}$ is not desirable if we do not want any enhanced symmetry.
} 
space and $E_{a b}$ is the matrix with $a b$-entry being one and all others being zeros. Similarly, $\left\{\widetilde{\beta}_{\alpha}-\widetilde{\beta}_{\beta}=\omega\right\}$ corresponds to simple roots $\left\{\delta_{\alpha}-\delta_{\beta}\right\}$ of $A_{M-1}$ so that $\widetilde{Z}_{\alpha \beta}$ take values in $\mathbb{C} E_{\alpha \beta}$.

We consider the case in which there is no enhanced gauge symmetry with $(N-1)$ different values of $\beta_{a}$ and $(M-1)$ different values of $\widetilde{\beta}_{\alpha}$.

In contrast to the Lie algebra, there are many inequivalent simple root systems in the basic Lie superalgebra consisting of the even roots

$$
\Delta_{\overline{0}}=\left\{\epsilon_{a}-\epsilon_{b} \mid 1 \leq a \neq b \leq N\right\} \cup\left\{\delta_{\alpha}-\delta_{\beta} \mid 1 \leq \alpha \neq \beta \leq M\right\}
$$

and the odd roots

$$
\Delta_{\overline{1}}=\left\{ \pm\left(\epsilon_{a}-\delta_{\alpha}\right) \mid 1 \leq a \leq N, 1 \leq \alpha \leq M\right\}
$$

where $\left\{\epsilon_{1}, \cdots, \epsilon_{N} ; \delta_{1}, \cdots, \delta_{M}\right\}$ is the basis of the root space with the bilinear forms $\left(\epsilon_{a}, \epsilon_{b}\right)=$ $\delta_{a b},\left(\delta_{\alpha}, \delta_{\beta}\right)=-\delta_{\alpha \beta}$ and $\left(\epsilon_{a}, \delta_{\alpha}\right)=0$. All the simple root systems are given by [38]

$$
\Pi= \pm\left\{\epsilon_{1}-\epsilon_{2}, \epsilon_{2}-\epsilon_{3}, \cdots, \epsilon_{s_{1}}-\delta_{1}, \delta_{1}-\delta_{2}, \cdots, \delta_{t_{1}}-\epsilon_{s_{1}+1}, \cdots\right\}
$$

up to the Weyl equivalence where $S=\left\{s_{1}<s_{2}<\cdots\right\}$ and $T=\left\{t_{1}<t_{2}<\cdots\right\}$ are two increasing sequences. Correspondingly $\left\{\beta_{a}-\beta_{b}\right\},\left\{\widetilde{\beta}_{\alpha}-\widetilde{\beta}_{\beta}\right\},\left\{\beta_{a}-\widetilde{\beta}_{\alpha}\right\}$ and $\left\{\widetilde{\beta}_{\alpha}-\beta_{a}\right\}$ admit different configurations for non-trivial valued $Z_{a b}, \widetilde{Z}_{\alpha \beta}, A_{a \alpha}$ and $B_{\alpha a}$.

According to the configuration (3.48), the Gauss law conditions (3.10)-(3.13) reduce to

$$
\begin{aligned}
{\left[Z, Z^{\dagger}\right]_{a b}+A_{a \alpha} A_{\alpha a}^{\dagger}-B_{a \alpha}^{\dagger} B_{\alpha a}+\kappa(N-M) \delta_{a N} \delta_{b N} } & =\kappa \delta_{a b}, \\
{[\widetilde{Z}, \widetilde{Z}]_{\alpha \beta}-A_{\alpha a}^{\dagger} A_{a \beta}+B_{\alpha a} B_{a \beta}^{\dagger} } & =\kappa \delta_{\alpha \beta}, \\
Z_{a b} B_{b \beta}^{\dagger}-B_{a \alpha}^{\dagger} \widetilde{Z}_{\alpha \beta}-Z_{a b}^{\dagger} A_{b \beta}+A_{a \alpha} \widetilde{Z}_{\alpha \beta}^{\dagger} & =0, \\
B_{\alpha a} Z_{a b}^{\dagger}-\widetilde{Z}_{\alpha \beta}^{\dagger} B_{\beta b}+\widetilde{Z}_{\alpha \beta} A_{\beta b}^{\dagger}-A_{\alpha a}^{\dagger} Z_{a b} & =0
\end{aligned}
$$

and the trace conditions (3.14), (3.15) and the supertrace condition (3.16) become

$$
\begin{aligned}
\sum_{a, \alpha}\left(A_{a \alpha} A_{\alpha a}^{\dagger}+B_{\alpha a} B_{a \alpha}^{\dagger}\right)+|x|^{2}-y^{\dagger} y & =\kappa N, \\
\sum_{a, \alpha}\left(A_{a \alpha} A_{\alpha a}^{\dagger}+B_{\alpha a} B_{a \alpha}^{\dagger}\right) & =\kappa M, \\
|x|^{2}-y^{\dagger} y & =\kappa(N-M) .
\end{aligned}
$$

Let us take, for some integer $r$ with $1 \leq r \leq M \leq N$,

$$
\begin{aligned}
& \beta_{a}= \begin{cases}(N+r+1-2 a) \omega & a=1, \cdots, r \\
(N-a) \omega & a=r+1, \cdots, N\end{cases} \\
& \widetilde{\beta}_{\alpha}= \begin{cases}(N+r-2 \alpha) \omega & \alpha=1, \cdots, r \\
(N+M+r-\alpha) \omega & \alpha=r+1, \cdots, M\end{cases}
\end{aligned}
$$


for which we have distinct values for $\beta_{a}, a=1, \cdots, N$ and $\widetilde{\beta}_{\alpha}, \alpha=1, \cdots, M$ as we are considering the case with no enhanced symmetry. These diagonal parts correspond to the set of roots

$\Delta_{\overline{0}}=\left\{\epsilon_{r+1}-\epsilon_{r+2}, \epsilon_{r+2}-\epsilon_{r+3}, \cdots, \epsilon_{N-1}-\epsilon_{N}\right\} \cup\left\{\delta_{r+1}-\delta_{r+2}, \delta_{r+2}-\delta_{r+3}, \cdots, \delta_{M-1}-\delta_{M}\right\}$, $\Delta_{\overline{1}}=\left\{\epsilon_{1}-\delta_{1}, \epsilon_{2}-\delta_{2}, \cdots, \epsilon_{r}-\delta_{r}\right\} \cup\left\{\delta_{M+1}-\epsilon_{1}, \delta_{1}-\epsilon_{2}, \delta_{2}-\epsilon_{3}, \cdots, \delta_{r}-\epsilon_{r+1}\right\}$.

The number of available independent components is $(N-r-1)+(M-r-1)+r+(r+1)=$ $N+M-1$. According to (3.66), the component fields of supermatrix $\widehat{Z}$ take general forms as

$$
\begin{aligned}
Z_{a b} & =Z_{a(a+1)}, & a & =r+1, \cdots, N-1, \\
\widetilde{Z}_{\alpha \beta} & =\widetilde{Z}_{\alpha(\alpha+1)}, & \alpha & =r+1, \cdots, M-1, \\
A_{a \alpha} & =A_{\alpha \alpha} \delta_{a \alpha}, & a & =1, \cdots, r, \\
B_{\alpha a} & =B_{\alpha(\alpha+1)} \delta_{a(\alpha+1)}+B_{M 1} \delta_{\alpha M} \delta_{a 1}, & \alpha & =1, \cdots, r .
\end{aligned}
$$

Then the Gauss law constraints (3.59) become

$$
\begin{aligned}
A_{11} A_{11}^{\dagger} & =B_{1 M}^{\dagger} B_{M 1}+\kappa, & & \\
A_{a a} A_{a a}^{\dagger} & =B_{a(a-1)}^{\dagger} B_{(a-1) a}+\kappa, & & a=2, \cdots, r, \\
\left|Z_{(r+1)(r+2)}\right|^{2} & =B_{(r+1) r}^{\dagger} B_{r(r+1)}+\kappa, & & \\
\left|Z_{a(a+1)}\right|^{2} & =\left|Z_{(a-1) a}\right|^{2}+\kappa, & & a=r+2, \cdots, \\
\left|Z_{(N-1) N}\right|^{2} & =\kappa(N-M-1) . & &
\end{aligned}
$$

and we find that

$$
\left|Z_{a(a+1)}\right|^{2}=\kappa(a-M)
$$

for $a=r+1, \cdots, N-1$. Due to the positivity of the equation (3.74), it follows that $r \geq M-1$ and so $r$ must be either $M-1$ or $M$.

\section{Parallel polarized vortex-antivortex state}

Let us consider the case for $r=M-1$, in which the configuration (3.66) reduces to

$$
\begin{aligned}
& \beta_{a}= \begin{cases}(N+M-2 a) \omega & a=1, \cdots, M-1 \\
(N-a) \omega & a=M, \cdots, N\end{cases} \\
& \widetilde{\beta}_{\alpha}= \begin{cases}(N+M-2 \alpha) \omega & \alpha=1, \cdots, M-1 \\
(N+M-1) \omega & \alpha=M\end{cases}
\end{aligned}
$$

and the corresponding simple root system is

$$
\begin{aligned}
& \Delta_{\overline{0}}=\left\{\epsilon_{M}-\epsilon_{M+1}, \epsilon_{M+2}-\epsilon_{M+2}, \cdots, \epsilon_{N-1}-\epsilon_{N}\right\} \\
& \Delta_{\overline{1}}=\left\{\epsilon_{1}-\delta_{1}, \epsilon_{2}-\delta_{2}, \cdots, \epsilon_{M-1}-\delta_{M-1}\right\} \cup\left\{\delta_{M+1}-\epsilon_{1}, \delta_{1}-\epsilon_{2}, \delta_{2}-\epsilon_{3}, \cdots, \delta_{M-1}-\epsilon_{M}\right\} .
\end{aligned}
$$


Thus the classical configuration does not admit non-trivial values for $\widetilde{Z}$. From the configurations (3.74) and the Gauss law conditions (3.71) one finds that

$$
B_{M(M-1)}^{\dagger} B_{(M-1) M}=-\kappa .
$$

Then the second set (3.60) of the Gauss law constraints become

$$
\begin{aligned}
-A_{\alpha \alpha}^{\dagger} A_{\alpha \alpha}+B_{\alpha(\alpha+1)} B_{(\alpha+1) \alpha}^{\dagger} & =\kappa, \quad \alpha=1, \cdots, M-2, \\
A_{(M-1)(M-1)}^{\dagger} A_{(M-1)(M-1)} & =0, \\
B_{M 1} B_{1 M}^{\dagger} & =\kappa .
\end{aligned}
$$

Combining these with (3.69)-(3.73) and (3.74), we obtain

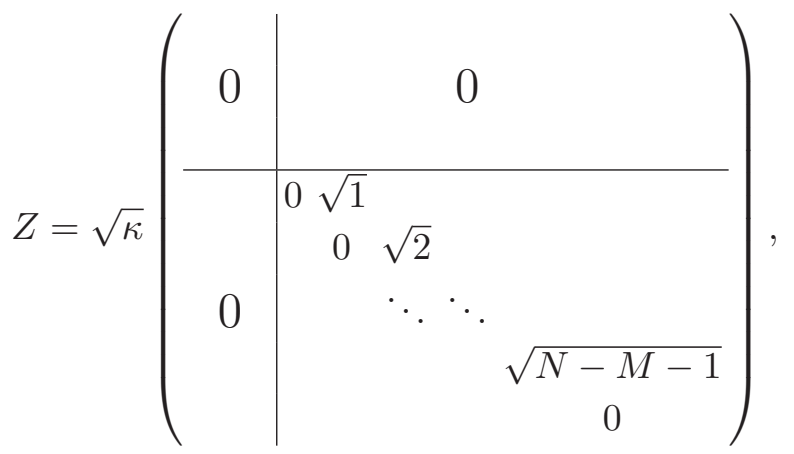

$$
\begin{aligned}
& B=\left(\begin{array}{ccccc|c}
0 & B_{12} & & & \\
\vdots & & B_{23} & & \\
& & & \ddots & & 0 \\
0 & & & & B_{(M-1) M} &
\end{array}\right) \\
& \widetilde{Z}=0 \text {, } \\
& \phi=\left(\begin{array}{c}
0 \\
0 \\
\vdots \\
\vdots \\
0 \\
x
\end{array}\right), \\
& A=0, \\
& \psi=\left(\begin{array}{c}
0 \\
0 \\
\vdots \\
\vdots \\
0 \\
y
\end{array}\right)
\end{aligned}
$$

so that

$$
\begin{aligned}
B_{(\alpha+1) \alpha}^{\dagger} B_{\alpha(\alpha+1)} & =-\kappa, & B_{1 M}^{\dagger} B_{M 1}=-\kappa, \\
|x|^{2}-y^{\dagger} y & =\kappa(N-M) . &
\end{aligned}
$$

The fermionic Gauss law conditions (3.61) and (3.62) hold for the above static configurations. 


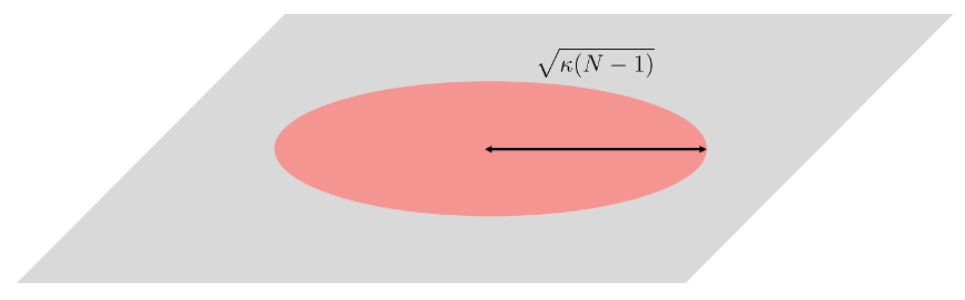

Figure 3. A quantum droplet of radius $\sqrt{\kappa(N-1)}$ formed by $N$ electrons on the plane.

In order to interpret our classical solutions (3.81) and (3.82), let us firstly consider a special case for $M=0$ where the supermatrix field $\widehat{Z}$ becomes the ordinary matrix $N \times N$ matrix $Z$ and $\widetilde{Z}=0, A=0$ and $B=0$. The non-trivial field configurations are given by

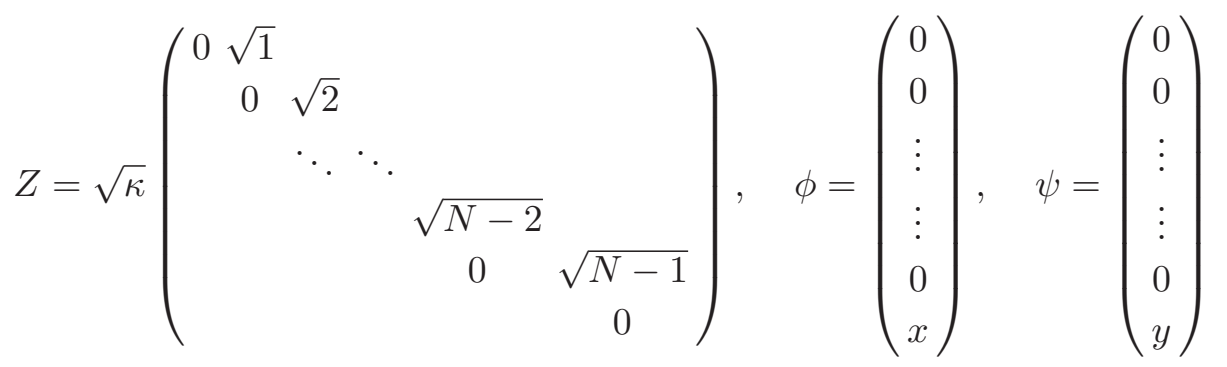

and we have the constraint

$$
|x|^{2}-y^{\dagger} y=\kappa N
$$

In this case, the gauge symmetry is an ordinary $\mathrm{U}(N)$ symmetry, but we still have a supergroup $\mathrm{SU}(p \mid q)$ flavour symmetry. We have already presented the generalized Calogero model for this case in section 3.1, but here we find the classical ground state. In the particular case when $q=0$, the supervector fields become $\phi=\sqrt{\kappa N}(0,0, \cdots, 1)^{T}, \psi=0$ so that (3.84) can be uniquely solved and our configuration is exactly same as the unique classical ground state in [1].

One can obtain a physical interpretation of the resulting configurations in the description of the fractional quantum Hall effect [1]. The solution (3.83) corresponds to the round quantum Hall droplet (see figure 3). The radius squared of the disk formed by $N$ electrons or $N$ vortices is given by the maximum eigenvalue of $Z^{\dagger} Z$

$$
R^{2}=\kappa(N-1)
$$

The total energy is given by

$$
E=\omega \operatorname{Tr}\left(Z^{\dagger} Z\right)=\kappa \omega \frac{N(N-1)}{2}
$$

and depends on the size of the system consisting of $N$ vortices.

As discussed in [1], the non-zero values $x$ and $y$ which absorb the anomaly of the commutators are required to realize the finite droplet and they are interpreted as 


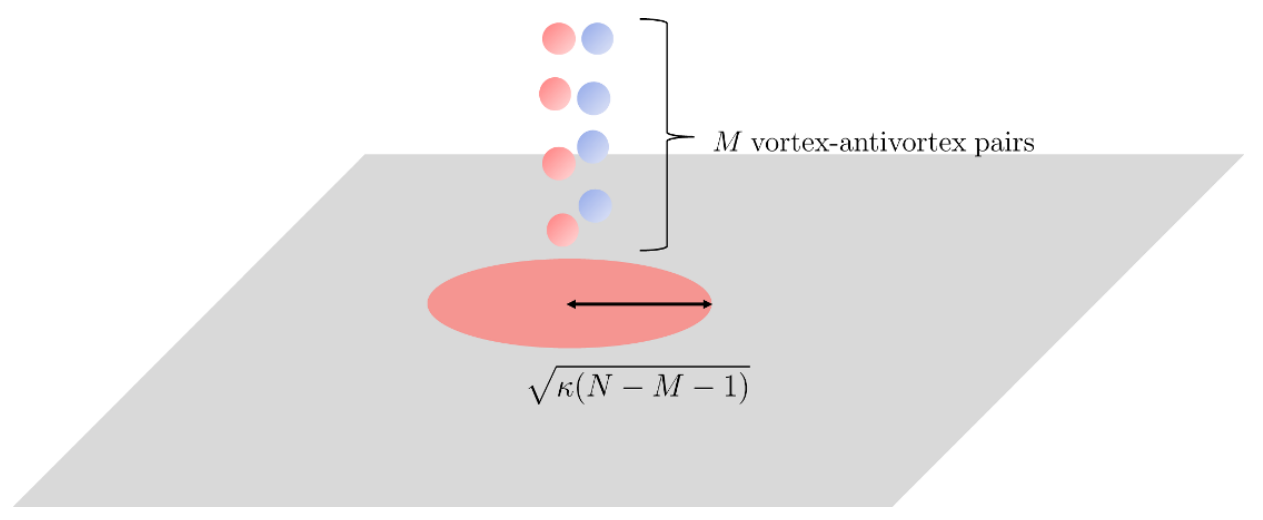

Figure 4. Parallel polarized vortex-antivortex pair ground state. $(N-M)$ vortices form a droplet of radius $\sqrt{\kappa(N-M)} . M$ vortex-antivortex pairs are created and the cores of vortices and antivortices approach each other on spiraling orbits and meet in the center.

boundary terms. In general, when $p+q>1$ such terms may be associated to certain additional internal degrees of freedom in the system. For example, when we consider multi-layered two-dimensional systems, e.g. multi-layered graphene (with $q=0$ ), they correspond to the so-called valley degeneracies, which label $p$ multi-layers of vortices in our case. Also note that in the solutions above with $p=q=1$ the $\mathrm{SU}(p \mid q)$ symmetry acts on $x$ and $y$ (preserving $x^{2}-y^{\dagger} y$ ) while all other fields are invariant (noting that $z=0$ for these solutions).

Now for a general case with $M \neq 0$ the radius squared of the disk formed by electrons is given by the maximum eigenvalue of $Z^{\dagger} Z$

$$
R^{2}=\kappa(N-M-1) .
$$

This indicates that $(N-M)$ electrons form a round disk of area $\pi \kappa(N-M-1)$. The total energy is

$$
E=\omega \operatorname{Tr}\left(Z^{\dagger} Z+B^{\dagger} B\right)=\kappa \omega\left[\frac{(N-M)(N-M-1)}{2}-M\right] .
$$

The first term is the energy of $(N-M)$ electrons or vortices as in (3.86). An interesting result is the second term with negative contribution to the energy. This is the interaction energy of vortices and antivortices. As we have argued in section 2.2.2, this indicates the negative binding energy of parallel polarized vortex-antivortex pairs (see figure 4). Accordingly this classical configuration is expected to be associated with $M$ vortex-antivortex pairs with parallel polarization.

2. Antiparallel vortex-antivortex ground state

Next consider the case of $r=M$. The configuration (3.66) is given by

$$
\begin{aligned}
\beta_{a} & = \begin{cases}(N+M+1-2 a) \omega & a=1, \cdots, M \\
(N-a) \omega & a=M+1, \cdots, N\end{cases} \\
\widetilde{\beta}_{\alpha} & =(N+M-2 \alpha) \omega .
\end{aligned}
$$


In these configurations all the values of $\beta_{a}$ and $\widetilde{\beta}_{\alpha}$ are different as required for no enhanced symmetry. As in the previous case, the configurations (3.89) admit $(N-$ $M-1)+0+M+M=N+M-1$ non-trivial components of supermatrix field $\widehat{Z}$, in which there are $2 M$ fermionic components. They correspond to the set of roots

$$
\begin{aligned}
& \Delta_{\overline{0}}=\left\{\epsilon_{M+1}-\epsilon_{M+2}, \epsilon_{M+2}-\epsilon_{M+3} \cdots, \epsilon_{N-1}-\epsilon_{N}\right\} \\
& \Delta_{\overline{1}}=\left\{\epsilon_{1}-\delta_{1}, \epsilon_{2}-\delta_{2}, \cdots, \epsilon_{M}-\delta_{M}\right\} \cup\left\{\delta_{1}-\epsilon_{2}, \delta_{2}-\epsilon_{3}, \cdots, \delta_{M}-\epsilon_{M+1}\right\} .
\end{aligned}
$$

In this case, the component fields of supermatrix $\widehat{Z}$ may take the form

$$
\begin{array}{rlrl}
Z & =Z_{a(a+1)}, & a & =M+1, \cdots, N-1, \\
\widetilde{Z} & =0, & \\
A_{a \alpha} & =A_{\alpha \alpha} \delta_{a \alpha}, & B_{\alpha a} & =B_{\alpha(\alpha+1)} \delta_{a(\alpha+1)}
\end{array}
$$

for $\alpha=1, \cdots, M$. Plugging the expressions (3.91) into the first set (3.59) of the Gauss law constraints, we get

$$
\begin{aligned}
A_{a a} A_{a a}^{\dagger} & =B_{a(a-1)}^{\dagger} B_{(a-1) a}+\kappa, & & a=1, \cdots, M, \\
\left|Z_{(M+1)(M+2)}\right|^{2} & =B_{(M+1) M}^{\dagger} B_{M(M+1)}+\kappa, & & \\
\left|Z_{a(a+1)}\right|^{2} & =\left|Z_{(a-1) a}\right|^{2}+\kappa, & & a=M+2, \cdots, N-1, \\
\left|Z_{(N-1) N}\right|^{2} & =\kappa(N-M-1) . & &
\end{aligned}
$$

Thus we find

$$
\begin{aligned}
\left|Z_{a(a+1)}\right|^{2} & =\kappa(a-M), \\
B_{M(M+1)} & =0
\end{aligned}
$$

for $a=M+1, \cdots, N-1$. From the Gauss law conditions (3.60) one finds

$$
A_{\alpha \alpha} A_{\alpha \alpha}^{\dagger}=B_{(\alpha+1) \alpha}^{\dagger} B_{\alpha(\alpha+1)}+\kappa
$$

where $\alpha=1, \cdots, M$. It then follows that

$$
B_{\alpha(\alpha+1)}=0, \quad A_{\alpha \alpha} A_{\alpha \alpha}^{\dagger}=\kappa .
$$


Putting all together, the classical solution is given by

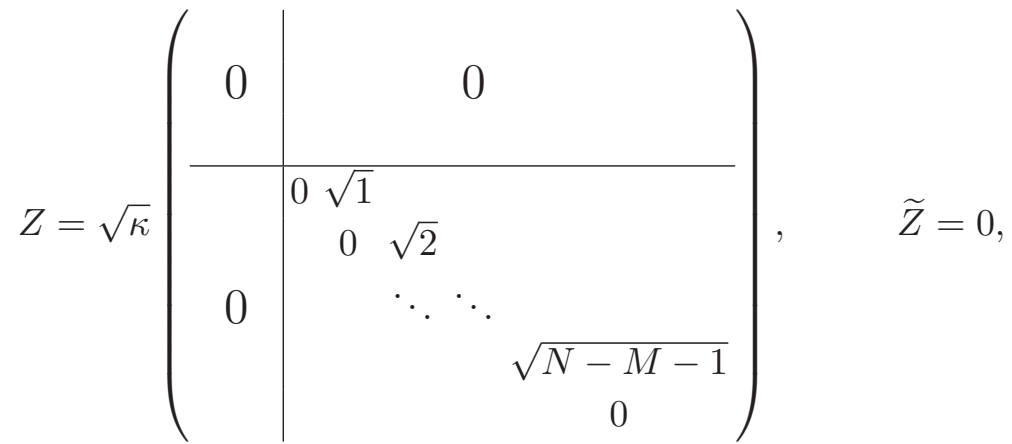$$
A=\left(\begin{array}{llll}
A_{11} & & & \\
& A_{22} & & \\
& & \ddots & \\
& & & A_{M M} \\
& & & \\
& & 0 &
\end{array}\right),
$$$$
B=0,
$$

$$
\phi=\left(\begin{array}{c}
0 \\
0 \\
\vdots \\
\vdots \\
0 \\
x
\end{array}\right)
$$

$$
\psi=\left(\begin{array}{c}
0 \\
0 \\
\vdots \\
\vdots \\
0 \\
y
\end{array}\right)
$$

so that

$$
A_{\alpha \alpha} A_{\alpha \alpha}^{\dagger}=\kappa, \quad|x|^{2}-y^{\dagger} y=\kappa(N-M) .
$$

The radius squared of the disk formed by electrons is given by the maximum eigenvalue of $Z^{\dagger} Z$

$$
R^{2}=\kappa(N-M-1) .
$$

Again this implies that $(N-M)$ vortices form a round disk of area $\pi \kappa(N-M-1)$. The total energy is

$$
E=\omega \operatorname{Tr}\left(Z^{\dagger} Z-A^{\dagger} A\right)=\kappa \omega\left[\frac{(N-M)(N-M-1)}{2}+M\right] .
$$

The first term is the energy of $(N-M)$ vortices as in (3.88) However, unlike the previous result (3.88), the second term has positive contributions to the energy. This would correspond to the positive energy of antiparallel polarized vortex-antivortex pairs, where the $M$ vortex-antivortex pairs lose the energy due to the emission of sound waves (see figure 5). Thus this classical solution would be the vortex-antivortex pairs of antiparallel polarization. 


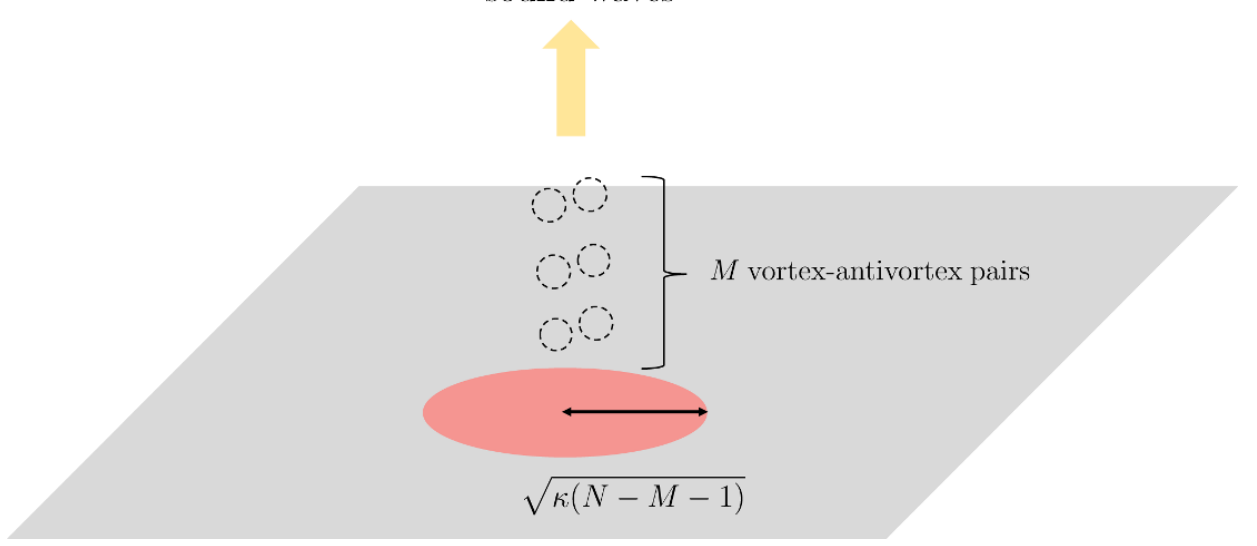

Figure 5. Antiparallel polarized vortex-antivortex pair ground state. $(N-M)$ electrons form a droplet of radius $\sqrt{\kappa(N-M-1)}$ on the plane. The annihilation of $M$ vortex-antivortex pairs releases the energy via the emission of sound wave.

\section{Quantum states}

\subsection{Quantization}

The elements of supermatrix $\widehat{Z}$ and the components of supervectors $\widehat{\Phi}$ become operators upon quantization. The action (2.11) specifies the canonical commutation relations

$$
\begin{aligned}
{\left[Z_{a b}, Z_{c d}^{\dagger}\right] } & =\delta_{a d} \delta_{b c}, & {\left[\widetilde{Z}_{\alpha \beta}, \widetilde{Z}_{\gamma \delta}^{\dagger}\right] } & =-\delta_{\alpha \delta} \delta_{\beta \gamma}, \\
\left\{A_{a \beta}, A_{\gamma d}^{\dagger}\right\} & =-\delta_{a d} \delta_{\beta \gamma}, & \left\{B_{\alpha b}, B_{c \delta}^{\dagger}\right\} & =\delta_{\alpha \delta} \delta_{b c}, \\
{\left[\phi_{a i}, \phi_{j b}^{\dagger}\right] } & =\delta_{i j} \delta_{a b}, & {\left[\widetilde{\phi}_{\alpha \lambda}, \widetilde{\phi}_{\rho \beta}^{\dagger}\right] } & =-\delta_{\lambda \rho} \delta_{\alpha \beta}, \\
\left\{\psi_{a \lambda}, \psi_{\rho b}^{\dagger}\right\} & =-\delta_{\lambda \rho} \delta_{a b}, & \left\{\widetilde{\psi}_{\alpha i}, \widetilde{\psi}_{j \beta}^{\dagger}\right\} & =\delta_{i j} \delta_{\alpha \beta},
\end{aligned}
$$

which can also be expressed in terms of superbrackets as

$$
\begin{gathered}
{\left[\widehat{Z}_{A B}, \widehat{Z}_{C D}^{\dagger}\right]_{S} \equiv \widehat{Z}_{A B} \widehat{Z}_{C D}^{\dagger}-(-1)^{(A+B)(C+D)} \widehat{Z}_{C D}^{\dagger} \widehat{Z}_{A B}=(-1)^{B} \delta_{A D} \delta_{B C},} \\
{\left[\widehat{\Phi}_{A I}, \widehat{\Phi}_{J B}^{\dagger}\right]_{S} \equiv \widehat{\Phi}_{A I} \widehat{\Phi}_{J B}^{\dagger}-(-1)^{(I+A)(J+B)} \widehat{\Phi}_{J B}^{\dagger} \widehat{\Phi}_{A I}=(-1)^{I} \delta_{I J} \delta_{A B} .}
\end{gathered}
$$

When $M=0$ and $q=0,(4.1)$ simplifies to

$$
\left[Z_{a b}, Z_{c d}^{\dagger}\right]=\delta_{a d} \delta_{b c}, \quad\left[\phi_{a i}, \phi_{j b}^{\dagger}\right]=\delta_{a b} \delta_{i j} .
$$

Given the canonical commutation relations (4.4), the quantization of the matrix ChernSimons model $(2.23)$ is performed in $[1,2]$ by introducing a reference state $|0\rangle$ that obeys

$$
Z_{a b}|0\rangle=\phi_{i}|0\rangle=0,
$$

and by acting on $|0\rangle$ with $Z^{\dagger}$ and $\varphi_{i}^{\dagger}$. When requiring that all physical states satisfy the Gauss law constraints there are operator ordering ambiguities. They are fixed as

$$
:\left[Z, Z^{\dagger}\right]:+\sum_{i=1}^{p} \phi_{i} \phi_{i}^{\dagger}=\kappa \mathbb{I}_{N} .
$$


Combining the commutation relations (4.4) and the trace part of the constraint (4.6), we find that

$$
\sum_{a=1}^{N} \sum_{i=1}^{p} \phi_{i a}^{\dagger} \phi_{a i}=(\kappa-p) N .
$$

This means that all physical states have charge $(\kappa-p)$ under the $\mathrm{U}(1) \subset \mathrm{U}(N)$. Alternatively it demands that all physical states involve $(\kappa-p) N$ copies of $\phi^{\dagger}$. Meanwhile the traceless part of the constraint (4.6) demands that they are $\mathrm{SU}(N)$ singlets.

However, in order to perform the quantization for the canonical commutation relations (4.1), one needs to determine which operators are realized by multiplication and which operators by differentiation on the quantum states. This prescription is called the polarization, which leads to the division of the phase space into coordinates and momenta. Here we encounter the issue on quantization due to the non-trivial polarization. There exist two polarizations which include the proposed quantization (4.5) in the ordinary matrix Chern-Simons model $[1,2]$.

1. Holomorphic polarization

Let us choose a polarization by introducing a reference state $|0\rangle$ that obeys

$$
\widehat{Z}_{A B}|0\rangle=\widehat{\Phi}_{A I}|0\rangle=0
$$

and construct the Hilbert space by acting on $|0\rangle$ with $\widehat{Z}_{A B}^{\dagger}$ and $\widehat{\Phi}_{I A}^{\dagger}$.

Due to the minus signs for the $\widetilde{\phi}, \psi, \widetilde{Z}$ and $A$ quantization conditions, we define the following number operators to count the number of each type of creation operator acting on the reference state:

$$
\begin{aligned}
N_{Z} & =\operatorname{Tr}\left(Z^{\dagger} Z\right), & & N_{\widetilde{Z}}=-\operatorname{Tr}\left(\widetilde{Z}^{\dagger} \widetilde{Z}\right), \\
N_{A} & =-\operatorname{Tr}\left(A^{\dagger} A\right), & & N_{B}=\operatorname{Tr}\left(B^{\dagger} B\right), \\
N_{\phi} & =\operatorname{Tr}\left(\phi^{\dagger} \phi\right), & & N_{\widetilde{\phi}}=-\operatorname{Tr}\left(\widetilde{\phi}^{\dagger} \widetilde{\phi}\right), \\
N_{\psi} & =-\operatorname{Tr}\left(\psi^{\dagger} \psi\right), & & N_{\widetilde{\psi}}=\operatorname{Tr}\left(\widetilde{\psi}^{\dagger} \widetilde{\psi}\right) .
\end{aligned}
$$

We can also define total number operators

$$
\begin{aligned}
& N_{\widehat{Z}}=N_{Z}+N_{B}+N_{\widetilde{Z}}+N_{A}=\operatorname{Str}\left(\widehat{Z}^{\dagger} \widehat{Z}\right), \\
& N_{\widehat{\Phi}}=N_{\phi}+N_{\widetilde{\phi}}+N_{\psi}+N_{\widetilde{\psi}}=(-1)^{I} \widehat{\Phi}_{I A}^{\dagger} \widehat{\Phi}_{A I} .
\end{aligned}
$$

Then the Hamiltonian is

$$
\begin{aligned}
H & =\omega \operatorname{Tr}\left(Z^{\dagger} Z+B^{\dagger} B-\widetilde{Z}^{\dagger} \widetilde{Z}-A^{\dagger} A\right) \\
& \equiv \omega\left(N_{Z}+N_{B}+N_{\tilde{Z}}+N_{A}\right) \equiv \omega N_{\widehat{Z}}
\end{aligned}
$$

which is manifestly non-negative. 
The system is a free set of bosonic and fermionic oscillators, subject to the Gauss law constraints on physical states. From the form of the Hamiltonian, we see that the ground state is the physical state with the minimal total $\widehat{Z}$ number, irrespective of contributions from $\widehat{\Phi}$.

To analyze the Gauss law constraints, we can just replace the expressions (3.10)-(3.13) with the corresponding operators expressions. There is the question of normal ordering. This is only relevant for the diagonal constraints, i.e. the diagonal parts of (3.10) and (3.11). Following [10] we can normal order the terms coming from $\widehat{Z}$. Choosing to do this or not to do this is equivalent to shifting the value of $\kappa$ by $(N-M)$. E.g. taking the trace of (3.10) without normal ordering gives the constraint

$$
N_{\phi}+N_{\psi}+N_{A}-N_{B}=N(\kappa-N+M-p+q)
$$

whereas if we had normal ordered $\widehat{Z}$ terms we would get

$$
N_{\phi}+N_{\psi}+N_{A}-N_{B}=N(\kappa-p+q) .
$$

The latter expression is more convenient when taking a large $N, M$ limit. Similarly, we could normal order the terms from $\widehat{\Phi}$ but this would just result in a shift of $\kappa$ by $(p-q)$ and we are considering those to be fixed in the large $N, M$ limit. These possibilities can all be encoded in a relation between $\kappa$ appearing the the action and $k$ defined so that in the quantum Gauss law constraints (3.10) and (3.11) we just take (3.10) and (3.11) to be completely normal ordered and replace $\kappa$ with $k$.

Now, taking the trace of (3.10) gives

$$
N_{\phi}+N_{\psi}+N_{A}-N_{B}=N k
$$

while taking the trace of (3.11) gives

$$
-N_{\widetilde{\phi}}-N_{\widetilde{\psi}}+N_{A}-N_{B}=M k .
$$

Taking the difference of these equations gives

$$
N_{\widehat{\Phi}} \equiv N_{\phi}+N_{\psi}+N_{\widetilde{\phi}}+N_{\widetilde{\psi}}=(N-M) k
$$

which is the supergroup analogue of (4.7).

2. Super polarization

Taking account into the superbracket

$$
\begin{aligned}
\widehat{Z}_{A B} \widehat{Z}_{C D}^{\dagger}-(-1)^{(A+B)(C+D)} \widehat{Z}_{C D}^{\dagger} \widehat{Z}_{A B} & =(-1)^{B} \delta_{A D} \delta_{B C} \\
& =(-1)^{C} \delta_{A D} \delta_{B C}
\end{aligned}
$$

and identifying the annihilation operator $\widehat{Z}_{A B}$ or $\widehat{Z}_{C D}^{\dagger}$ as we have $\left[a, a^{\dagger}\right]=1$ or $\left\{a, a^{\dagger}\right\}=1$, we can consider the reference state defined by

$$
\begin{aligned}
(-1)^{B+1} \widehat{Z}_{A B}|0\rangle & =\widehat{Z}_{A B}|0\rangle, \\
(-1)^{C} \widehat{Z}_{C D}^{\dagger}|0\rangle & =\widehat{Z}_{C D}^{\dagger}|0\rangle .
\end{aligned}
$$


Similarly we could impose

$$
\begin{aligned}
(-1)^{I+1} \widehat{\Phi}_{A I}|0\rangle & =\widehat{\Phi}_{A I}|0\rangle, \\
(-1)^{I} \widehat{\Phi}_{I A}^{\dagger}|0\rangle & =\widehat{\Phi}_{I A}^{\dagger}|0\rangle .
\end{aligned}
$$

We call this procedure of quantization the super polarization. We will leave more detailed investigation of this super polarization to future work.

\subsection{Quantum ground states}

We will first review the quantum ground states for the models without supergroup symmetries. These states can be constructed as (a power of) a determinant of a matrix of operators acting on the reference state. This motivates similar constructions in the more general supergroup case, generally involving superdeterminants. However, there are different candidate states. The states constructed all solve the Gauss Law constraints, but we do not have a proof that there are no physical states with lower energy. In addition, one complication is that in some cases the construction may give the zero state due to the possibility of constructing nilpotent operators in the supergroup case. This results in the possibility that a construction may produce the ground state for low enough values of $k$, but for larger $k$ will simply produce the zero state.

\subsubsection{Determinant states}

In the analysis of the ordinary matrix Chern-Simons theory (2.23), which is regarded as our model for $M=0$ and $q=0$, the quantum physical states are constructed [1,2]. Let us firstly review the construction. For $p=1$ the ground state can be constructed by acting with $k N$ copies of $\phi^{\dagger}$ while keeping the number of $Z^{\dagger}$ to a minimum. In addition, the ground state should be the $\mathrm{SU}(N)$ singlet. Defining a baryon operator by

$$
\mathcal{B} \equiv \epsilon^{a_{1} \cdots a_{N}}\left(Z^{l_{1}} \phi\right)_{a_{1}}^{\dagger} \cdots\left(Z^{l_{N}} \phi\right)_{a_{N}}^{\dagger}
$$

where all the exponents $l_{a}$ are distinct because of the antisymmetrization factor $\epsilon^{a_{1} \cdots a_{N}}$, the baryon generator with the lowest energy

$$
\mathcal{B}_{\min } \equiv \epsilon^{a_{1} \cdots a_{N}}\left(Z^{0} \phi\right)_{a_{1}}^{\dagger}\left(Z^{1} \phi\right)_{a_{2}}^{\dagger} \cdots\left(Z^{N-1} \phi\right)_{a_{N}}^{\dagger}
$$

gives the ground state as $k$ multiple $\mathcal{B}_{\text {min }}$ 's $[1,5]$

$$
\mid \text { ground }\rangle_{k}=\left[\epsilon^{a_{1} \cdots a_{N}}\left(Z^{0} \phi\right)_{a_{1}}^{\dagger}\left(Z^{1} \phi\right)_{a_{2}}^{\dagger} \cdots\left(Z^{N-1} \phi\right)_{a_{N}}^{\dagger}\right]^{k}|0\rangle,
$$

which carries $k N$ copies of $\phi^{\dagger}$ and $k$ charges of the $\mathrm{U}(1) \subset \mathrm{U}(N)$. Note that the baryon generator (4.26) with the lowest energy is in one to one correspondence with the Vandermonde determinant

$$
\Delta=\epsilon^{a_{1} \cdots a_{N}} z_{a_{1}}^{0} \cdots z_{a_{N}}^{N-1}=\prod_{a<b}\left(z_{a}-z_{b}\right) .
$$


When $N$ is divisible by $p \geq 2$, the ground state is also uniquely determined. One can build up the $\mathrm{SU}(p)$ singlet by collecting $p$ creation operators $\phi_{i}^{\dagger}$ into the baryon operator

$$
\mathcal{B}(r)_{a_{1} \cdots a_{p}}^{\dagger} \equiv \epsilon^{i_{1} \cdots i_{p}}\left(Z^{r} \phi\right)_{i_{1} a_{1}}^{\dagger} \cdots\left(Z^{r} \phi\right)_{i_{p} a_{p}}^{\dagger} .
$$

This is a singlet under the $\mathrm{SU}(p)$ transforming as the $p$-th antisymmetric representation of the $\mathrm{U}(N)$ gauge symmetry group. To construct the $\mathrm{SU}(N)$ singlet with $k N$ charge of the $\mathrm{U}(1) \subset \mathrm{U}(N)$, we furthermore collect $N / p$ baryon operators $\mathcal{B}(0)_{a_{1} \cdots a_{p}}^{\dagger}, \mathcal{B}(1)_{a_{p+1} \cdots a_{2 p}}^{\dagger}, \cdots$, $\mathcal{B}\left(\frac{N}{p}-1\right)_{a_{N-p+1} \cdots a_{N}}^{\dagger}$ by introducing a baryon of baryons

$$
\mathcal{B}_{\min } \equiv \epsilon^{a_{1} \cdots a_{N}} \mathcal{B}(0)_{a_{1} \cdots a_{p}}^{\dagger} \mathcal{B}(1)_{a_{p+1} \cdots a_{2 p}}^{\dagger} \cdots \mathcal{B}\left(\frac{N}{p}-1\right)_{a_{N-p+1} \cdots a_{N}}^{\dagger}
$$

and find the ground state $[2]$

$$
\mid \text { ground }\rangle_{k}=\left[\epsilon^{a_{1} \cdots a_{N}} \mathcal{B}(0)_{a_{1} \cdots a_{p}}^{\dagger} \mathcal{B}(1)_{a_{p+1} \cdots a_{2 p}}^{\dagger} \cdots \mathcal{B}\left(\frac{N}{p}-1\right)_{a_{N-p+1} \cdots a_{N}}^{\dagger}\right]^{k}|0\rangle
$$

whose energy is

$$
E=\omega k p \sum_{r=0}^{\frac{N}{p}-1} r=\omega k \frac{N(N-p)}{2 p} .
$$

Note that the ground state can also be written in the form

$$
\mid \text { ground }\rangle_{k}=\left(\operatorname{Det}\left(S_{I a}\right)\right)^{k}|0\rangle
$$

where the elements of the $N \times N$ matrix are

$$
S_{I a}=\left[\phi^{\dagger}\left(Z^{\dagger}\right)^{r}\right]_{i a}
$$

with the notation

$$
I=r p+i, i \in\{1,2, \ldots, p\}, r \in\left\{0,1, \ldots, \frac{N}{p}-1\right\} .
$$

When $N$ is not divisible by $p \geq 2$, there is no $\mathrm{SU}(p)$ singlet ground state. Let us express $N=m p+n, m, n \in \mathbb{Z}_{>0}$. Then the ground state is constructed as

$$
\begin{aligned}
\mid \text { ground }\rangle_{k}= & \prod_{l=1}^{k}\left[\epsilon^{a_{1} \cdots a_{N}} \mathcal{B}(0)_{a_{1} \cdots a_{p}}^{\dagger} \mathcal{B}(1)_{a_{p+1} \cdots a_{2 p}}^{\dagger} \cdots \mathcal{B}(m-1)_{a_{N-p-n+1} \cdots a_{N-n}}^{\dagger}\right. \\
& \left.\left(Z^{m} \phi_{i_{(l, 1)}}\right)_{a_{N-n+1}}^{\dagger} \cdots\left(Z^{m} \phi_{i_{(l, n)}}\right)_{a_{N}}^{\dagger}\right]|0\rangle,
\end{aligned}
$$

where the indices $i_{(l, \alpha)}$ with $l=1, \cdots, k \alpha=1, \cdots, n$ label the degenerate ground states. The ground state energy is

$$
E=\omega k\left(p \sum_{r=0}^{m-1} r+n m\right)=\omega k\left[\frac{p m(m-1)}{2}+m n\right] .
$$




\subsubsection{Superdeterminant states - case 1}

Now we look for solutions to the Gauss law constraints, allowing non-zero $M$ and $q$. As reviewed in section 4.2.1 in the case of ordinary Lie groups, the ground state was given by a determinant acting on the reference state. As may be expected the generalization to supergroups requires the use of a superdeterminant. Necessarily this is a rather formal expression since superdeterminants are not polynomial functions of the matrix elements. However, for now we simply show that at a formal level this gives a solution of the Gauss law constraints. Explicitly, we conjecture a potential class of ground states given by

$$
|S G g s 1\rangle=S^{k}|0\rangle \text {. }
$$

Here we have defined

$$
S \equiv \operatorname{Sdet}\left(S_{\mathcal{I} A}\right)
$$

where the elements of the $(N+M) \times(N+M)$ matrix are defined as follows:

$$
\begin{aligned}
S_{I A} & =\left[\widehat{\Phi}^{\dagger}\left(\widehat{Z}^{\dagger}\right)^{r}\right]_{i A}, \\
S_{\Lambda A} & =\left[\widehat{\Phi}^{\dagger}\left(\widehat{Z}^{\dagger}\right)^{r}\right]_{\lambda A} .
\end{aligned}
$$

In these expressions $I$ label the $N$ even components while $\Lambda$ label the $M$ odd components indexed by $\mathcal{I}$. Although not explicitly labelled as such, the exponents $r$, and indices $i$ and $\lambda$ in each expression are determined by $I$ or $\Lambda$. Specifically, (taking for now the simplest case where $N$ is a multiple of $p$ and $M$ is a multiple of $q$ ) we have

$$
\begin{aligned}
& I=r p+i, \quad i \in\{1,2, \ldots, p\}, r \in\left\{0,1, \ldots, \frac{N}{p}-1\right\}, \\
& \Lambda=r q+\lambda, \lambda \in\{1,2, \ldots, q\}, r \in\left\{0,1, \ldots, \frac{M}{q}-1\right\} .
\end{aligned}
$$

The normal ordered Gauss law constraints are given by

$$
\left.\left(\widehat{G}_{A B}-k \delta_{A B}\right) \mid \text { phys }\right\rangle=0
$$

where

$$
\widehat{G}_{A B}=(-1)^{(A+C)(C+B)} \widehat{Z}_{C B}^{\dagger} \widehat{Z}_{A C}-\widehat{Z}_{A C}^{\dagger} \widehat{Z}_{C B}+(-1)^{(I+A)(I+B)} \widehat{\Phi}_{I B}^{\dagger} \widehat{\Phi}_{A I}
$$

is the quantum Gauss law constraint operator.

It is then straightforward to check that the state $|S G g s 1\rangle$ defined in equation (4.38) is indeed a physical state. One method is to note that superdeterminants can be written as ratios of ordinary determinants, and then the commutation relations can be used to find the commutators of $\widehat{Z}_{A B}$ and $\widehat{\Phi}_{A I}$ with $S$. E.g. using the standard even/odd split form of a supermatrix we have

$$
\operatorname{Sdet}\left(\begin{array}{ll}
A & B \\
C & D
\end{array}\right)=\operatorname{det}\left(A-B D^{-1} C\right)(\operatorname{det}(D))^{-1} .
$$


Note that while this is invariant under $\mathrm{U}(N \mid M)$ transformations, it is not invariant under $\mathrm{SU}(p \mid q)$ transformations except in the case where $N / p=M / q$, since only then are all $p+q$ components $\left[\widehat{\Phi}^{\dagger}\left(\widehat{Z}^{\dagger}\right)^{r}\right]_{i A}$ and $\left[\widehat{\Phi}^{\dagger}\left(\widehat{Z}^{\dagger}\right)^{r}\right]_{\lambda A}($ for each $A$ ) included in the superdeterminant expression for all values of $r$.

A short calculation shows that (relative to the reference state $|0\rangle$ ) the potential ground state has energy

$$
E_{S G g s 1}=\omega k\left(\frac{N(N-p)}{2 p}-\frac{M(M-q)}{2 q}\right)
$$

This reproduces the ground state energy (4.32) for $M=q=0$. We expect that this gives the ground state energy in some more general cases with non-zero $M$ and $q$. In the case where $N$ is not a multiple of $p$ or $M$ is not a multiple of $q$, a superdeterminant generalisation of (4.36) will lead to an expression for the potential ground state energy generalising (4.37). However, there will be cases where different constructions give physical states with lower energy, particularly for small values of $k$. We consider explicitly the case of $M=0$ but $q \neq 0$, in which case, at least for $k=1$, the construction in this section does not give the ground state energy. We now consider this possibility and later generalize that construction to $M \neq 0$.

\subsubsection{Analysis for $\mathrm{U}(N)$}

In the case where $M=0$ but $q \neq 0$, we note that the previous construction could be used. However, the state would not involve any of the fermionic creation operators $\psi_{\lambda a}^{\dagger}$. This motivates us to consider another possibility to construct the candidate ground state from the reference state. To do this, we consider the candidate ground state

$$
\left|G S_{M=0}\right\rangle \equiv S^{k}|0\rangle
$$

We have defined

$$
S \equiv \sqrt{\operatorname{Det}\left(\left(S^{T} S\right)_{a b}\right)}
$$

where

$$
\left(S^{T} S\right)_{a b}=S_{a \mathcal{I}}^{T} S_{\mathcal{I} b} \equiv S_{\mathcal{I} a} S_{\mathcal{I} b}
$$

and the elements of the $N \times N$ matrix $S_{\mathcal{I} a}$ are:

$$
\begin{aligned}
S_{I a} & =\left[\widehat{\Phi}^{\dagger}\left(Z^{\dagger}\right)^{r}\right]_{i a}, \\
S_{\Lambda a} & =\left[\widehat{\Phi}^{\dagger}\left(Z^{\dagger}\right)^{r}\right]_{\lambda a} .
\end{aligned}
$$

Clearly $S^{k}=\left(S^{2}\right)^{k / 2}$ is polynomial for even $k$, while for odd $k$ we do need to consider the square root in (4.49). In these expressions $I$ label even components while $\Lambda$ label odd components indexed by $\mathcal{I}$. Although not explicitly labelled as such, the exponents $r$, and 
indices $i$ and $\lambda$ in each expression are determined by $I$ or $\Lambda$. Specifically, taking for now the simplest case where $N$ is a multiple of $p+q$, we have

$$
\begin{aligned}
& I=r p+i, \quad i \in\{1,2, \ldots, p\}, r \in\left\{0,1, \ldots, \frac{N}{p+q}-1\right\}, \\
& \Lambda=r q+\lambda, \lambda \in\{1,2, \ldots, q\}, r \in\left\{0,1, \ldots, \frac{N}{p+q}-1\right\} .
\end{aligned}
$$

Note that for $q=0$ this again reproduces the previous determinant construction of the ground state.

The normal ordered Gauss law constraints are given by

$$
\left(G_{a b}-k \delta_{a b}\right)|\mathrm{phys}\rangle=0
$$

where

$$
G_{a b}=Z_{c b}^{\dagger} Z_{a c}-Z_{a c}^{\dagger} Z_{c b}+(-1)^{I} \widehat{\Phi}_{I b}^{\dagger} \widehat{\Phi}_{a I}
$$

is the quantum Gauss law constraint operator.

It is then straightforward to check that the state $\left|G S_{M=0}\right\rangle$ defined in equation (4.48) is indeed a physical state. Another short calculation shows that (relative to the reference state $|0\rangle)$ the potential ground state has energy

$$
E_{G S, M=0}=\omega k \frac{N(N-p-q)}{2(p+q)} .
$$

Note that the above claim for the potential ground state energy is made on the assumption that $\left|G S_{M=0}\right\rangle$ is a normalizable state. In fact this state will vanish for sufficiently large $k$. To see this simply note that if we set all the fermions to zero the matrix $S_{\mathcal{I} a}$ would have $q N /(p+q)$ rows of zeros, so consequently $S=0$. Reintroducing the fermions we see that all terms in $S^{2}$ must involve a product of at least $q N /(p+q)$ fermions. Since we only have $q N$ independent fermionic components of $\widehat{\Phi}_{a \lambda}$ we see that certainly $S^{k}=0$ for all $k>p+q$. In fact, we may have $S^{k}=0$ for lower values of $k$. It is not clear what the interpretation of this bound is but it does indicate a richer structure appears for the supergroup models. The simplest case to explore the issue of the constructions producing the zero state is $N=2$ and $p=q=1$.

We find a ground state for $k=1$ described by

$$
S \equiv \operatorname{Det}\left(\begin{array}{cc}
\phi_{1}^{\dagger} & \phi_{2}^{\dagger} \\
\psi_{1}^{\dagger} & \psi_{2}^{\dagger}
\end{array}\right)=\phi_{1}^{\dagger} \psi_{2}^{\dagger}-\phi_{2}^{\dagger} \psi_{1}^{\dagger}
$$

which is clearly generically non-vanishing. We have dropped the redundant labels $i=1$ and $\lambda=1$ in this example.

However, it is easy to check that $S^{2}=0$ so we have only found the ground state for $k=1$ and it indeed has energy zero as expected. Now, in this example we can try to explicitly construct the ground state for $k=2$. However, the result shown below is that 
no such state exists, i.e. that for $k=2$ there is no extremal energy eigenstate arising as a polynomial of creation operators acting on the reference state.

First note that the Gauss law constraints can be expressed as conditions on $S$

$$
k \delta_{a b} S=\left[G_{a b}, S\right] \equiv\left(Z_{c b}^{\dagger} \frac{\partial}{\partial Z_{c a}^{\dagger}}-Z_{a c}^{\dagger} \frac{\partial}{\partial Z_{b c}^{\dagger}}+\phi_{b}^{\dagger} \frac{\partial}{\partial \phi_{a}^{\dagger}}+\psi_{b}^{\dagger} \frac{\partial}{\partial \psi_{a}^{\dagger}}\right) S .
$$

It is a simple task to check that there is no zero energy state for $k \geq 2$, at least assuming it is constructed as a polynomial of the creation operators acting on the reference state. In this case the requirement of zero energy is simply that no $Z^{\dagger}$ operators are used to create the state. Then the two Gauss law constraints

$$
\left[G_{11}, S\right]=\left[G_{22}, S\right]=k S
$$

impose the constraint that $S$ must have the form

$$
S=\left(\phi_{1}^{\dagger}\right)^{k-1}\left(\phi_{2}^{\dagger}\right)^{k-1}\left(c_{00} \phi_{1}^{\dagger} \phi_{2}^{\dagger}+c_{01} \psi_{1}^{\dagger} \phi_{2}^{\dagger}+c_{10} \phi_{1}^{\dagger} \psi_{2}^{\dagger}+c_{11} \psi_{1}^{\dagger} \psi_{2}^{\dagger}\right)
$$

Now we still have to impose the constraints

$$
\left[G_{12}, S\right]=\left[G_{21}, S\right]=0
$$

but clearly this means that $G_{12}$ and $G_{21}$ must annihilate the term with coefficient $c_{00}$, and separately the term with coefficient $c_{11}$. This is only possible if $c_{00}=0$ and (except for $k=1) c_{11}=0$. Then we are left with

$$
\begin{aligned}
& {\left[G_{12}, S\right]=c_{01}(k-1)\left(\phi_{1}^{\dagger}\right)^{k-2}\left(\phi_{2}^{\dagger}\right)^{k+1} \psi_{1}^{\dagger}+\left(c_{01}+k c_{10}\right)\left(\phi_{1}^{\dagger}\right)^{k-1}\left(\phi_{2}^{\dagger}\right)^{k} \psi_{2}^{\dagger},} \\
& {\left[G_{21}, S\right]=c_{10}(k-1)\left(\phi_{1}^{\dagger}\right)^{k+1}\left(\phi_{2}^{\dagger}\right)^{k-2} \psi_{2}^{\dagger}+\left(c_{10}+k c_{01}\right)\left(\phi_{1}^{\dagger}\right)^{k}\left(\phi_{2}^{\dagger}\right)^{k-1} \psi_{1}^{\dagger} .}
\end{aligned}
$$

These equations have no solution unless $S=0$ or $k=1$.

We can relax the condition that the energy vanishes, but explicit calculation shows that there is still no solution for the case $k=2$. Of course, the superdeterminant construction of the previous section provides a formal solution, but not polynomial in the creation operators acting on the reference state.

\subsubsection{Superdeterminant states - case 2}

The previous considerations for $M=0$ lead to an alternative proposal for the ground states. We can define an $(N+M) \times(N+M)$ matrix with elements

$$
\begin{aligned}
S_{I A} & =\left[\widehat{\Phi}^{\dagger}\left(Z^{\dagger}\right)^{r}\right]_{i A}, \\
S_{\Lambda A} & =\left[\widehat{\Phi}^{\dagger}\left(Z^{\dagger}\right)^{r}\right]_{\lambda A} .
\end{aligned}
$$

Here the simplest construction is when $M+N$ is a multiple of $p+q$, in which case

$$
\begin{aligned}
& I=r p+i, \quad i \in\{1,2, \ldots, p\}, r \in\left\{0,1, \ldots, \frac{N+M}{p+q}-1\right\}, \\
& \Lambda=r q+\lambda, \lambda \in\{1,2, \ldots, q\}, r \in\left\{0,1, \ldots, \frac{N+M}{p+q}-1\right\} .
\end{aligned}
$$


Then we propose the potential ground states

$$
|S G g s 2\rangle \equiv S^{k}|0\rangle
$$

where

$$
S \equiv \sqrt{\operatorname{Sdet}\left(\left(S^{T} S\right)_{a b}\right)}
$$

and

$$
\left(S^{T} S\right)_{A B}=S_{A \mathcal{I}}^{T} S_{\mathcal{I} B} \equiv S_{\mathcal{I} A} S_{\mathcal{I} B}
$$

It is then straightforward to check that the state $|S G g s 2\rangle$ defined in equation (4.69) is indeed a physical state and that (relative to the reference state $|0\rangle)$ it has energy

$$
E_{S G g s 2}=\omega k \frac{(N-M)(N+M-p-q)}{2(p+q)} .
$$

Note that this state is by construction the same as the state $\left|G S_{M=0}\right\rangle$ defined in equation (4.48) in the case $M=0$, and it is also exactly the same as the state $|S G g s 1\rangle$ defined in equation (4.38) in the case where $N / p=M / q$. However, in general the states $|S G g s 1\rangle$ and $|S G g s 2\rangle$ are different, and it seems likely that the states $|S G g s 2\rangle$ are the better candidate ground states. One particular feature the states $|S G g s 2\rangle$ have is that (when $N+M$ is a multiple of $p+q$ ) they respect the $\mathrm{SU}(p \mid q)$ symmetry.

Finally, we note that when both superdeterminant constructions are compared (assuming $N / p, M / q$ and $(N+M) /(p+q)$ are all integer) the difference in energies is

$$
E_{S G g s 1}-E_{S G g s 2}=\frac{\omega k}{2}\left(q^{2} N^{2}-p^{2} M^{2}\right)
$$

which can be positive, negative or zero.

In terms of a potential relation to WZW models, as demonstrated for $M=0$ and $q=0[10]$ we must consider a generalization of the large- $N$ limit with fixed $p$ and now also fixed $q$. Two natural choices are to take $M=0$, or to scale $N$ and $M$ in the same ratio as $p: q$. In the $M=0$ case we believe $|S G g s 2\rangle$ is the ground state, while in the other case $|S G g s 1\rangle=|S G g s 2\rangle$.

\section{$5 \quad$ Kac-Moody algebra}

When $M=0, q=0$, it was demonstrated [10] that the matrix degrees of freedom lead to the affine Lie algebra $\widehat{\mathfrak{s u}}(p)$ in the large $N$ limit. We will firstly review the argument of [10]. Then we conjecture that the result generalizes to $q \neq 0$, leading to a $\widehat{\mathfrak{s u}}(p \mid q)$ current algebra. We show this in the case $M=0$ but expect it also holds in a large $N$ and large $M$ limit. 


\subsection{Affine Lie algebra}

In the case of $M=0, q=0$, i.e. $\mathrm{U}(N)$ and $\mathrm{SU}(p)$ symmetry, in [10] the current operators were defined as

$$
\begin{aligned}
& \widetilde{J}^{m}=\operatorname{Tr} Z^{m}, \\
& \widetilde{J}_{i j}^{m}=i\left(\phi_{i}^{\dagger} Z^{m} \phi_{j}-\frac{1}{p} \delta_{i j} \phi_{k}^{\dagger} Z^{m} \phi_{k}\right)
\end{aligned}
$$

with $i, j, k=1, \cdots, p$ and $m \geq 0$.

It is then straightforward to show that for $m$ and $n$ either both non-negative or both non-positive

$$
\left[\widetilde{J}_{i j}^{m}, \widetilde{J}_{k l}^{n}\right]=i\left(\delta_{i l} \widetilde{J}_{k j}^{m+n}-\delta_{k j} \widetilde{J}_{i l}^{m+n}\right)
$$

For $m<0$ the negative graded currents are defined by

$$
\widetilde{J}_{i j}^{m}=\widetilde{J}_{j i}^{m \mid \dagger} .
$$

Now the commutator of a current at positive level with one at negative level is more involved as it requires evaluating commutators of powers of $Z$ with powers of $Z^{\dagger}$. In particular, for non-negative $m$ and $n$

$$
\begin{aligned}
{\left[\widetilde{J}_{i j}^{m}, \widetilde{J}_{k l}^{-n}\right] } & =\left[\phi_{i}^{\dagger} Z^{m} \phi_{j}, \phi_{k}^{\dagger} Z^{\dagger n} \phi_{l}\right] \\
& =\delta_{j k} \phi_{i}^{\dagger} Z^{m} Z^{\dagger n} \phi_{l}-\delta_{i l} \phi_{k}^{\dagger n} Z^{m} \phi_{j}+\phi_{i a}^{\dagger} \phi_{k b}\left[Z_{a c}^{m}, Z_{b d}^{\dagger n}\right] \phi_{j c} \phi_{l d}
\end{aligned}
$$

Additionally the current algebra is expected only when acting on physical states, so the Gauss law constraint must be imposed. In fact, the $\widehat{\mathfrak{s u}}(p)$ current algebra is only correctly reproduced in a large $N$ limit.

The combination of imposing the Gauss law constraints and the large $N$ limit is carried out using knowledge of the classical and quantum ground states. Specifically, results such as $Z^{\dagger} \phi_{i}=0$ for classical ground states are taken to imply that this relation holds for all physical states (at least those with sufficiently low energy) to leading order in a $1 / N$ expansion. Using such considerations it is possible to identify the leading large $N$ behavior of various terms and retaining only the leading non-vanishing order in expressions greatly simplifies the results. The discussion of classical solutions is applicable to calculations of Poisson brackets, but this is expected to carry over to quantum commutation relations. We refer the reader to [10] for more details, although we also make some more detailed comparisons when generalizing the results to $q \neq 0$.

The result is that after a rescaling ${ }^{5}$ of the currents

$$
J_{i j}^{m}=\left(\frac{(\kappa) N}{p}\right)^{-\frac{|m|}{2}} \widetilde{J}_{i j}^{m}
$$

\footnotetext{
${ }^{5}$ Note that this rescaling is trivial in (5.3) as that equation is homogeneous provided $m$ and $n$ have the same sign.
} 
the Kac-Moody algebra is produced at leading large $N$ order

$$
\left[J_{i j}^{m}, J_{k l}^{n}\right] \sim i\left(\delta_{i l} J_{k j}^{m+n}-\delta_{k j} J_{i l}^{m+n}\right)+\kappa m\left(\delta_{j k} \delta_{i l}-\frac{1}{p} \delta_{i j} \delta_{k l} .\right) .
$$

Actually, this is the classical Poisson bracket result, but it was argued [10] to hold also for quantum commutators up to the replacement $\kappa \rightarrow k$ in the central term.

\subsection{Affine Lie superalgebra}

We now consider the case with $q \neq 0$ where we expect to get an affine Lie superalgebra. The case where $M=0$ is simpler than $M \neq 0$ although the calculations are similar, so we present this first and briefly comment on the large $M$ case in the next section.

We define the supertraceless (in the $I J$ indices) currents

$$
\begin{aligned}
J_{I J}^{m} & \equiv\left(\widehat{\Phi}_{I}^{\dagger} Z^{m} \widehat{\Phi}_{J}-\frac{(-1)^{M}}{p-q} \delta_{I J} \widehat{\Phi}_{M}^{\dagger} Z^{m} \widehat{\Phi}_{M}\right), \\
J_{I J}^{-m} & =\left(J_{J I}^{m}\right)^{\dagger}
\end{aligned}
$$

for $m \geq 0$. In defining the current, note that it is the supertrace, not the trace, which is invariant under $\mathrm{SU}(p \mid q)$ transformations $\widehat{\Phi}_{I} \rightarrow \widehat{\Phi}_{K} M_{K I}$. Also, $\delta_{I J}$ and not $\eta_{I J} \equiv(-1)^{I} \delta_{I J}$ is invariant under the same transformation as $\widehat{\Phi}_{I}^{\dagger} Z^{m} \widehat{\Phi}_{J}$.

We now calculate explicitly for $m \geq 0$ and $n \geq 0$, using the quantization conditions. The superbracket of currents is easily calculated to give

$$
\begin{aligned}
{\left[J_{I J}^{m}, J_{K L}^{n}\right]_{S} } & \equiv J_{I J}^{m} J_{K L}^{n}-(-1)^{(I+J)(K+L)} J_{K L}^{n} J_{I J}^{m} \\
& =\eta_{J K} J_{I L}^{m+n}-(-1)^{(I+J)(K+L)} \eta_{I L} J_{K J}^{m+n}
\end{aligned}
$$

Now we calculate (up to terms ensuring supertracelessness in $I J$ and in $K L$ )

$$
\begin{aligned}
{\left[J_{I J}^{m}, J_{K L}^{-n}\right]_{S}=} & (-1)^{J K} \widehat{\Phi}_{I a}^{\dagger} \widehat{\Phi}_{K b}^{\dagger}\left[\left(Z^{m}\right)_{a c},\left(Z^{\dagger n}\right)_{b d}\right] \widehat{\Phi}_{J c} \widehat{\Phi}_{L d} \\
& +\eta_{J K} \widehat{\Phi}_{I}^{\dagger}\left(Z^{\dagger n} Z^{m}+\left[Z^{m}, Z^{\dagger n}\right]\right) \widehat{\Phi}_{L}-(-1)^{(I+J)(K+L)} \eta_{I L} \widehat{\Phi}_{K}^{\dagger} Z^{\dagger n} Z^{m} \widehat{\Phi}_{J}
\end{aligned}
$$

which generalizes (2.7) in [10].

We can analyze this similarly to [10]. E.g. [10] (2.10) onwards becomes

$$
\begin{aligned}
& \widehat{\Phi}_{I}^{\dagger}\left[Z^{m}, Z^{\dagger n}\right] \widehat{\Phi}_{L} \\
& \sim-\sum_{r=0}^{m-1} \sum_{s=0}^{n-1}\left(\widehat{\Phi}_{I}^{\dagger} Z^{r} Z^{\dagger s} \widehat{\Phi}_{M}\right)\left(\widehat{\Phi}_{M}^{\dagger} Z^{\dagger n-1-s} Z^{m-1-r} \widehat{\Phi}_{L}\right)+\kappa n \sum_{r=0}^{m-1} \widehat{\Phi}_{I}^{\dagger} Z^{r} Z^{\dagger n-1} Z^{m-1-r} \widehat{\Phi}_{L}, \\
& \widehat{\Phi}_{I a}^{\dagger} \widehat{\Phi}_{K b}^{\dagger}\left[\left(Z^{m}\right)_{a c},\left(Z^{\dagger n}\right)_{b d}\right] \widehat{\Phi}_{J c} \widehat{\Phi}_{L d} \\
& \sim \sum_{r=0}^{m-1} \sum_{s=0}^{n-1}(-1)^{(J+K) L}\left(\widehat{\Phi}_{I}^{\dagger} Z^{r} Z^{\dagger s} \widehat{\Phi}_{L}\right)\left(\widehat{\Phi}_{K}^{\dagger} Z^{\dagger n-1-s} Z^{m-1-r} \widehat{\Phi}_{J}\right)
\end{aligned}
$$


Now we introduce some notation to split $\widehat{\Phi}_{I}^{\dagger} Z^{r} Z^{\dagger s} \widehat{\Phi}_{J}$ and $\widehat{\Phi}_{I}^{\dagger} Z^{\dagger r} Z^{s} \widehat{\Phi}_{J}$ into their supertrace and supertraceless parts

$$
\begin{aligned}
X_{I J}^{r, s} & \equiv \widehat{\Phi}_{I}^{\dagger} Z^{r} Z^{\dagger s} \widehat{\Phi}_{J}-\delta_{I J} X^{r, s}, \\
X^{r, s} & \equiv \frac{(-1)^{M}}{p-q} \widehat{\Phi}_{M}^{\dagger} Z^{r} Z^{\dagger s} \widehat{\Phi}_{M}, \\
Y_{I J}^{r, s} & \equiv \widehat{\Phi}_{I}^{\dagger} Z^{\dagger r} Z^{s} \widehat{\Phi}_{J}-\delta_{I J} Y^{r, s}, \\
Y^{r, s} & \equiv \frac{(-1)^{M}}{p-q} \widehat{\Phi}_{M}^{\dagger} Z^{\dagger r} Z^{s} \widehat{\Phi}_{M}
\end{aligned}
$$

so that the terms above with four $\Phi$ s can be written as

$$
\begin{aligned}
\Theta \sim & \sum_{r=0}^{m-1} \sum_{s=0}^{n-1}\left((-1)^{(J+K) L+J K}\left(X_{I L}^{r, s}+\delta_{I L} X^{r, s}\right)\left(Y_{K J}^{n-1-s, m-1-r}+\delta_{K} Y^{n-1-s, m-1-r}\right)\right. \\
& \left.-\eta_{J K}\left(X_{I M}^{r, s}+\delta_{I M} X^{r, s}\right)\left(Y_{M L}^{n-1-s, m-1-r}+\delta_{M L} Y^{n-1-s, m-1-r}\right)\right) \\
= & \sum_{r=0}^{m-1} \sum_{s=0}^{n-1}\left((-1)^{(J+K) L+J K} \delta_{I L} X^{r, s} Y_{K J}^{n-1-s, m-1-r}-\eta_{J K} X^{r, s} Y_{I L}^{n-1-s, m-1-r}\right. \\
& \left.+(-1)^{(J+K) L+J K} X_{I L}^{r, s} Y_{K J}^{n-1-s, m-1-r}-\eta_{J K} X_{I M}^{r, s} Y_{M L}^{n-1-s, m-1-r}\right)
\end{aligned}
$$

noting that all four terms containing $Y^{n-1-s, m-1-r}$ cancel. Now we need to apply the large $N$ limit with generalizations of Identities 1 and 2 in [10].

We apply the large $N$ limit as in [10]. The classical solutions for $M=0$ are similar in nature to those of [10] where also $q=0$. In particular the form of $Z$ is the same and $Z^{\dagger} \widehat{\Phi}_{I}=0$ in the ground state. We also assume this implies that $Z^{\dagger} \widehat{\Phi}_{I}$ is suppressed compared to the naive expectations based on the order of $Z^{\dagger}$ and $\widehat{\Phi}$ at large $N$. This means that the same results as in [10] hold, up to possible signs. In particular, to leading order in $N$

$$
\begin{aligned}
\sum_{r=0}^{m-1} \widehat{\Phi}_{I}^{\dagger} Z^{r} Z^{\dagger n-1} Z^{m-1-r} \widehat{\Phi}_{L} & \sim \frac{1}{p-q} \delta_{I L} \sum_{r=0}^{m-1}(-1)^{M} \widehat{\Phi}_{M}^{\dagger} Z^{r} Z^{\dagger n-1} Z^{m-1-r} \widehat{\Phi}_{M} \\
& \sim \frac{(-1)^{M}}{p-q} \delta_{I L} \widehat{\Phi}_{M}^{\dagger} Z^{\dagger n-1} Z^{m-1} \widehat{\Phi}_{M}
\end{aligned}
$$

Collecting terms we find

$$
\begin{aligned}
{\left[J_{I J}^{m}, J_{K L}^{-n}\right]_{S} \sim } & \sum_{r=0}^{m-1} \sum_{s=0}^{n-1}\left((-1)^{(J+K) L+J K} \delta_{I L} X^{r, s} Y_{K J}^{n-1-s, m-1-r}-\eta_{J K} X^{r, s} Y_{I L}^{n-1-s, m-1-r}\right. \\
& +(-1)^{(J+K) L+J K} X_{I L}^{r, s} Y_{K J}^{n-1-s, m-1-r}-\eta_{J K} X_{I M}^{r, s} Y_{M L}^{n-1-s, m-1-r} \\
& \left.+\kappa n \eta_{J K} \delta_{I L} Y^{n-1, m-1}+\eta_{J K} Y_{I L}^{n, m}-(-1)^{(I+J)(K+L)} \eta_{I L} Y_{K J}^{n, m}\right)
\end{aligned}
$$

The second line of (5.21) is subleading at large $N$ compared to the first line so we drop it from now on. Also, in the above sums over $r$ and $s$, the dominant terms come from $r=s=0$ since $Z^{\dagger} \widehat{\Phi}_{I}$ and $\widehat{\Phi}_{I}^{\dagger} Z$ are suppressed. 
Noting that the Gauss law constraints are

$$
\left[Z, Z^{\dagger}\right]+\widehat{\Phi}_{M} \widehat{\Phi}_{M}^{\dagger}=\kappa \mathcal{I}
$$

a slight generalization of the derivation of Identity 1 in appendix A of [10] (particularly (A.4) and the equation directly above it) gives

$$
\begin{aligned}
Y_{I L}^{n, n+m} & \sim\left(\frac{\kappa N}{p-q}\right)^{n} Y_{I L}^{0, m}+\widehat{\Phi}_{I}^{\dagger} \widehat{\Phi}_{J} \widehat{\Phi}_{J}^{\dagger} Z^{\dagger n-1} Z^{m-1+n} \widehat{\Phi}_{L} \\
& \sim\left(\frac{\kappa N}{p-q}\right)^{n} Y_{I L}^{0, m}+\left(\frac{\kappa N}{p-q}\right) Y_{I L}^{n-1, n+m-1}
\end{aligned}
$$

where the terms kept are those at leading order in $N$ and consistent with the fact that the expression is traceless. This can be rewritten as

$$
Y_{I L}^{n, n+m}-X^{0,0} Y_{I L}^{n-1, n+m-1} \sim\left(\frac{\kappa N}{p-q}\right)^{n} Y_{I L}^{0, m}
$$

Now the dominant terms in the first line of (5.21) are from the case $r=s=0$, and these combine with the last two terms in the final line in exactly the combination in (5.24) so we have in the case $m \geq n$

$$
\begin{aligned}
{\left[J_{I J}^{m}, J_{K L}^{-n}\right]_{S} \sim } & \eta_{J K}\left(\frac{\kappa N}{p-q}\right)^{n} Y_{I L}^{0, m-n}-(-1)^{(I+J)(K+L)} \eta_{I L}\left(\frac{\kappa N}{p-q}\right)^{n} Y_{K J}^{0, m-n} \\
& +\kappa n \eta_{J K} \delta_{I L} Y^{n-1, m-1}
\end{aligned}
$$

Now, rescaling the currents $J^{m}$ by a factor $(\kappa N /(p-q))^{-|m| / 2}$ we get

$$
\left[J_{I J}^{m}, J_{K L}^{-n}\right]_{S} \sim \eta_{J K} J_{I L}^{m-n}-(-1)^{(I+J)(K+L)} \eta_{I L} J_{K J}^{m-n}+\kappa n \eta_{J K} \delta_{I L} \delta_{m n}
$$

using a generalization of Identity 2 in [10] and noting that terms from $Y^{n-1, n-1}$ with $n \neq m$ are suppressed and so do not contribute to this result for large $N$.

Finally, we correct this expression to ensure it is supertraceless in both $I J$ and $K L$ so we have

$$
\left[J_{I J}^{m}, J_{K L}^{-n}\right]_{S} \sim \eta_{J K} J_{I L}^{m-n}-(-1)^{(I+J)(K+L)} \eta_{I L} J_{K J}^{m-n}+\kappa n\left(\eta_{J K} \delta_{I L}-\frac{1}{p-q} \delta_{I J} \delta_{K L}\right) \delta_{m n} .
$$

Presumably we would also have a quantum version of the generalization of Identity 2 in [10] which would have the effect of introducing a factor of $k / \kappa$ in the central term, in which case the final result would be

$$
\left[J_{I J}^{m}, J_{K L}^{-n}\right]_{S} \sim \eta_{J K} J_{I L}^{m-n}-(-1)^{(I+J)(K+L)} \eta_{I L} J_{K J}^{m-n}+k n\left(\eta_{J K} \delta_{I L}-\frac{1}{p-q} \delta_{I J} \delta_{K L}\right) \delta_{m n} .
$$




\subsection{Generalization to $\mathrm{U}(N \mid M)$}

We expect the results of the previous section for $M=0$ generalize to $M \neq 0$ provided we take a combined large $N$ and large $M$ limit. Specifically, we would expect that the natural limit is to take large $N / p=M / q$. As the arguments are essentially the same as in the previous section, we just present the definitions and result. We also note the decomposition under $\mathrm{U}(N) \times \mathrm{U}(M) \in \mathrm{U}(N \mid M)$ but calculations are most naturally carried out using superbrackets without such a decomposition.

For $p=q$ we need to take more care but otherwise we can propose the following supertraceless $\mathrm{SU}(p \mid q)$ supercurrents

$$
\widehat{J}_{I J}^{m}=i\left(\widehat{\Phi}_{I}^{\dagger} \widehat{Z}^{m} \widehat{\Phi}_{J}-\frac{(-1)^{K}}{p-q} \delta_{I J} \widehat{\Phi}_{K}^{\dagger} \widehat{Z}^{m} \widehat{\Phi}_{K}\right) \equiv\left(\begin{array}{cc}
\widehat{J}_{i j}^{m} & \widehat{J}_{i \kappa}^{m} \\
\widehat{J}_{\lambda j}^{m} & \widehat{J}_{\lambda \kappa}^{m}
\end{array}\right)
$$

Denoting elements of $\widehat{Z}^{m}$ by

$$
\widehat{Z}^{m}=\left(\begin{array}{cc}
\widehat{Z}_{B}^{m} & \widehat{Z}_{F}^{m} \\
\widehat{Z}_{\widetilde{F}}^{m} & \widehat{Z}_{\widetilde{B}}^{m}
\end{array}\right)
$$

one can express the currents (5.29) by

$$
\begin{aligned}
\widehat{J}_{i j}^{m}= & i\left[\phi_{i}^{\dagger} \widehat{Z}_{B}^{m} \phi_{j}+\widetilde{\psi}_{i}^{\dagger} \widehat{Z}_{\widetilde{F}}^{m} \phi_{j}+\phi_{i}^{\dagger} \widehat{Z}_{F}^{m} \widetilde{\psi}_{j}+\widetilde{\psi}_{i}^{\dagger} \widehat{Z}_{\widetilde{B}}^{m} \widetilde{\psi}_{j}\right]-\frac{i}{p-q} \delta_{i j} \widehat{K}^{m}, \\
\widehat{J}_{\lambda \kappa}^{m}= & i\left[\widetilde{\phi}_{\lambda}^{\dagger} \widehat{Z}_{\widetilde{B}}^{m} \widetilde{\phi}_{\kappa}+\psi_{\lambda}^{\dagger} \widehat{Z}_{F}^{m} \widetilde{\phi}_{\kappa}+\widetilde{\phi}_{\lambda}^{\dagger} \widehat{Z}_{\widetilde{F}}^{m} \psi_{\kappa}+\psi_{\lambda}^{\dagger} \widehat{Z}_{B}^{m} \psi_{\kappa}\right]-\frac{i}{p-q} \delta_{\lambda \kappa} \widehat{K}^{m}, \\
\widehat{J}_{i \kappa}^{m}= & i\left[\phi_{i}^{\dagger} \widehat{Z}_{B}^{m} \psi_{\kappa}+\widetilde{\psi}_{i}^{\dagger} \widehat{Z}_{\widetilde{F}}^{m} \psi_{\kappa}+\phi_{i}^{\dagger} \widehat{Z}_{F}^{m} \widetilde{\phi}_{\kappa}+\widetilde{\psi}_{i}^{\dagger} \widehat{Z}_{\widetilde{B}}^{m} \widetilde{\phi}_{\kappa}\right], \\
\widehat{J}_{\lambda j}^{m}= & i\left[\psi_{\lambda}^{\dagger} \widehat{Z}_{B}^{m} \phi_{j}+\widetilde{\phi}_{\lambda}^{\dagger} \widehat{Z}_{\widetilde{F}}^{m} \phi_{j}+\psi_{\lambda}^{\dagger} \widehat{Z}_{F}^{m} \widetilde{\psi}_{j}+\widetilde{\phi}_{\lambda}^{\dagger} \widehat{Z}_{B}^{m} \widetilde{\psi}_{j}\right], \\
\widehat{K}^{m}= & {\left[\phi_{k}^{\dagger} \widehat{Z}_{B}^{m} \phi_{k}+\widetilde{\psi}_{k}^{\dagger} \widehat{Z}_{\widetilde{F}}^{m} \phi_{k}+\phi_{k}^{\dagger} \widehat{Z}_{F}^{m} \widetilde{\psi}_{k}+\widetilde{\psi}_{k}^{\dagger} \widehat{Z}_{\widetilde{B}}^{m} \widetilde{\psi}_{k}\right] } \\
& -\left[\widetilde{\phi}_{\rho}^{\dagger} \widehat{Z}_{\widetilde{B}}^{m} \widetilde{\phi}_{\rho}+\psi_{\rho}^{\dagger} \widehat{Z}_{F}^{m} \widetilde{\phi}_{\rho}+\widetilde{\phi}_{\rho}^{\dagger} \widehat{Z}_{\widetilde{F}}^{m} \psi_{\rho}+\psi_{\rho}^{\dagger} \widehat{Z}_{B}^{m} \psi_{\rho}\right] .
\end{aligned}
$$

Assuming the large $N$ and now also large $M$ properties hold, along with generalizations of the classical and quantum identities described in the previous section, we will find the same affine Lie superalgebra result

$$
\left[\widehat{J}_{I J}^{m}, \widehat{J}_{K L}^{-n}\right]_{S} \sim\left(\eta_{J K} \widehat{J}_{I L}^{m-n}-(-1)^{(I+J)(K+L)} \eta_{I L} \widehat{J}_{K J}^{m-n}\right)+k m\left(\eta_{J K} \delta_{I L}-\frac{1}{p-q} \delta_{I J} \delta_{K L}\right) \delta_{m n}
$$

for large $N / p=M / q$. 


\section{Partition function}

\subsection{Definition}

In this section we study the spectrum of the system by computing the partition function of the supermatrix Chern-Simons model (2.1). Let us consider the modified Hamiltonian

$$
\begin{aligned}
H^{\prime}= & \omega \operatorname{Str}\left(\widehat{Z}^{\dagger} \widehat{Z}\right)-\sum_{I, A} \widehat{\mu}_{I}(-1)^{I} \widehat{\Phi}_{I A}^{\dagger} \Phi_{A I} \\
= & \omega \operatorname{Tr}\left(Z^{\dagger} Z+B^{\dagger} B-\widetilde{Z}^{\dagger} \widetilde{Z}-A^{\dagger} A\right) \\
& -\sum_{i=1}^{p} \sum_{a=1}^{N} \mu_{i} \phi_{i a}^{\dagger} \phi_{a i}+\sum_{\lambda=1}^{q} \sum_{\alpha=1}^{M} \widetilde{\mu}_{\lambda} \widetilde{\phi}_{\lambda \alpha}^{\dagger} \widetilde{\phi}_{\alpha \lambda}-\sum_{i=1}^{p} \sum_{\alpha=1}^{M} \mu_{i} \widetilde{\psi}_{i \alpha}^{\dagger} \widetilde{\psi}_{\alpha i}+\sum_{\lambda=1}^{q} \sum_{a=1}^{N} \widetilde{\mu}_{\lambda} \psi_{\lambda a}^{\dagger} \psi_{a \lambda} .
\end{aligned}
$$

Here we have introduced the chemical potential $-\sum \widehat{\mu}_{I}(-1)^{I} \widehat{\Phi}_{I A}^{\dagger} \widehat{\Phi}_{A I}$ where $\widehat{\mu}_{I}=\left\{\mu_{i}, \widetilde{\mu}_{\lambda}\right\}$ is a set of coupling constants. It counts the number of $\hat{Z}^{\dagger}$ and $\widehat{\Phi}_{I}^{\dagger}$ excitations with weights $\omega$ and $\mu_{I}$. When evaluated on the physical state $\mid$ phys $\rangle$, the modified Hamiltonian gives

$$
\left.\left.H^{\prime} \mid \text { phys }\right\rangle=\left(\omega N_{\widehat{Z}}-\sum_{i=1}^{p} \mu_{i} J_{i}-\sum_{\lambda=1}^{q} \widetilde{\mu}_{\lambda} \widetilde{J}_{\lambda}\right) \mid \text { phys }\right\rangle
$$

where

$$
N_{\widehat{Z}}=N_{Z}+N_{B}+N_{\widetilde{Z}}+N_{A}
$$

is the total number of excitations of $\widehat{Z}^{\dagger}$ and

$$
J_{i}=N_{\phi_{i}}+N_{\widetilde{\psi}_{i}}, \quad \widetilde{J}_{\lambda}=N_{\widetilde{\phi}_{\lambda}}+N_{\psi_{\lambda}}
$$

is the total number of excitations of fundamental fields $\phi_{i}$ and $\widetilde{\psi}_{i}$ and that of fundamental fields $\widetilde{\phi}_{\lambda}$ and $\psi_{\lambda}$. The partition function of the modified Hamiltonian is given by

$$
\mathcal{Z}(q, x, y)=\operatorname{Tr} e^{-\beta H^{\prime}}=\operatorname{Tr} q^{N_{\widehat{Z}}} \prod_{i=1}^{p} x_{i}^{J_{i}} \prod_{\lambda=1}^{q} y_{\lambda}^{\widetilde{J}_{\lambda}}
$$

where the trace is taken over the physical states $\mid$ phys $\rangle$ and $\beta$ is the inverse temperature. We have defined parameters $q:=e^{-\beta \omega}, x_{i}:=e^{\beta \mu_{i}}$ and $y_{\lambda}:=e^{\beta \widetilde{\mu}_{\lambda}}$.

To compute this partition function, we firstly collect all states and then project out the non-physical states by requiring that the physical states are gauge invariant so that they obey the Gauss law constraints. The Lie superalgebra $\mathfrak{u}(N \mid M)$ is a $\mathbb{Z}_{2}$-graded space $V$ decomposed into a direct sum of $\mathbb{Z}_{2}$-graded subspaces $V_{\overline{0}}$ and $V_{\overline{1}}$. As we have the supertrace form on $\mathfrak{u}(N \mid M)$, a supersymmetric bilinear form on $V$ is defined so that $V_{\overline{0}}$ and $V_{\overline{1}}$ are orthogonal and the restriction of the bilinear form to $V_{\overline{0}}$ is symmetric and to $V_{\overline{1}}$ is skewsymmetric. Identifying the Cartan subalgebra $\mathfrak{h}$ with the root space $\mathfrak{h}^{*}$ via this bilinear form, we have [38]

$$
\epsilon_{a}=E_{a a}, \quad \delta_{\alpha}=-E_{\alpha \alpha}
$$


where $\epsilon_{a}, a=1, \cdots, N$ and $\delta_{\alpha}, \alpha=1, \cdots, M$ are a basis of the root space $\mathfrak{h}^{*}$ while $E_{a b}$ and $E_{\alpha \beta}$ are the basis of the Cartan subalgebra $\mathfrak{h}$. Since (6.6) defines the gauge charges, the relative minus sign for the $\mathfrak{u}(M)$ subalgebra would require an additional sign to read off the correct $\mathrm{U}(1)$ charges for the $\mathrm{U}(M)$ symmetry from the related excitation modes.

We will focus on the holomorphic polarized quantization where the Hilbert space is constructed by acting with $\widehat{Z}_{A B}^{\dagger}$ and $\widehat{\Phi}_{I A}^{\dagger}$ on the reference states $|0\rangle$. All the physical states are characterized by the number operators $N_{Z}, N_{\widetilde{Z}}, N_{A}, N_{B}, N_{\phi_{i}}, N_{\widetilde{\phi}}, N_{\psi}$ and $N_{\widetilde{\psi}}$. Their quantum numbers $N_{\widehat{Z}}, J_{i}$ and $\widetilde{J}_{\lambda}$ appearing in the partition function (6.5) are determined from (6.3) and (6.4). Their gauge charges are determined from the trace parts (4.19) and (4.20) of the quantum Gauss law conditions by noting the relation (6.6). Let $\mathfrak{q}$ and $\widetilde{\mathfrak{q}}$ be the diagonal $\mathrm{U}(1)$ charges for $\mathrm{U}(1)^{N} \subset \mathrm{U}(N)$ and $\mathrm{U}(1)^{M} \subset \mathrm{U}(M)$ of the associated excitation modes respectively. Then they read

$$
\begin{aligned}
& \mathfrak{q}[Z]=0, \quad \mathfrak{q}[\widetilde{Z}]=0, \quad \mathfrak{q}[A]=1, \quad \mathfrak{q}[B]=-1 \\
& \mathfrak{q}[\phi]=1, \quad \mathfrak{q}[\widetilde{\phi}]=0, \quad \mathfrak{q}[\psi]=1, \quad \mathfrak{q}[\widetilde{\psi}]=0 \\
& \widetilde{\mathfrak{q}}[Z]=0, \quad \widetilde{\mathfrak{q}}[\widetilde{Z}]=0, \quad \widetilde{\mathfrak{q}}[A]=-1, \quad \tilde{\mathfrak{q}}[B]=1 \\
& \widetilde{\mathfrak{q}}[\phi]=0, \quad \widetilde{\mathfrak{q}}[\widetilde{\phi}]=1, \quad \widetilde{\mathfrak{q}}[\psi]=0, \quad \widetilde{\mathfrak{q}}[\widetilde{\psi}]=1
\end{aligned}
$$

In the following we will introduce $\omega_{a}$ and $\widetilde{\omega}_{\alpha}$ as the fugacity parameters for each Cartan element of the gauge symmetries $\mathrm{U}(1)^{N} \subset \mathrm{U}(N)$ and $\mathrm{U}(1)^{M} \subset \mathrm{U}(M)$ respectively. Taking account into these charges and fugacity parameters, we can collect all the contributions to the partition function as follows:

\section{1. $\mathcal{Z}_{\widehat{Z}}$}

Supermatrix field $\widehat{Z}_{A B}$ consists of bosonic fields $Z_{a b}, \widetilde{Z}_{\alpha \beta}$ and fermionic fields $A_{a \beta}$ and $B_{\alpha b}$. According to (6.3), each of the associated excitations carries quantum number $N_{\widehat{Z}}=1$. In addition, these component fields have two units of gauge charges as they involve two gauge indices. According to (6.7) and (6.9), $A_{a \beta}$ and $B_{\alpha b}$ have quantum numbers of $\frac{\omega_{a}}{\widetilde{\omega}_{\beta}}$ and $\frac{\widetilde{\omega}_{\alpha}}{\omega_{b}}$ respectively. Although the total gauge charges of $Z_{a b}$ and $\widetilde{Z}_{\alpha \beta}$ are zero, as we are now turning on the gauge fugacity parameter for each of the Cartan elements, $Z_{a b}$ and $\widetilde{Z}_{\alpha \beta}$ carry quantum numbers of $\frac{\omega_{a}}{\omega_{b}}$ and $\frac{\widetilde{\omega}_{\alpha}}{\widetilde{\omega}_{\beta}}$. The contribution to the partition function from the operators $\widehat{Z}_{A B}$ is given by

$$
\mathcal{Z}_{\widehat{Z}}=\prod_{a, b=1}^{N} \frac{1}{1-q \frac{\omega_{a}}{\omega_{b}}} \prod_{\alpha, \beta=1}^{M} \frac{1}{1-q \frac{\widetilde{\omega}_{\alpha}}{\widetilde{\omega}_{\beta}}} \prod_{c=1}^{N} \prod_{\gamma=1}^{M}\left(1+q \frac{\widetilde{\omega}_{\gamma}}{\omega_{c}}\right)\left(1+q \frac{\omega_{c}}{\widetilde{\omega}_{\gamma}}\right)
$$

where the first two factors come from the bosonic fields $Z_{a b}, \widetilde{Z}_{\alpha \beta}$ and the latter two from the fermionic fields $A_{a \beta}$ and $B_{\alpha b}$.

2. $\mathcal{Z}_{\widehat{\Phi}}$

The operators $\widehat{\Phi}_{A I}$ involve $\phi_{a i}, \widetilde{\phi}_{\alpha \lambda}, \psi_{a \lambda}$ and $\widetilde{\psi}_{\alpha i}$. While $\phi_{a i}$ and $\widetilde{\psi}_{\alpha i}$ carry quantum numbers $J_{i}=1, \widetilde{\phi}_{\alpha \lambda}$ and $\psi_{a \lambda}$ have quantum numbers $\widetilde{J}_{\lambda}=1$. Unlike the supermatrix 
field, these fields are labelled by a single gauge index. As seen from the gauge charges (6.8) and (6.10), $\phi_{a i}$ and $\psi_{a \lambda}$ have quantum numbers of $\omega_{a}$, while $\widetilde{\phi}_{\alpha \lambda}$ and $\widetilde{\psi}_{\alpha i}$ have those of $\widetilde{\omega}_{\alpha}$. The contribution to the partition function from the operators $\widehat{\Phi}_{A I}$ is given by

$$
\mathcal{Z}_{\widehat{\Phi}}=\prod_{a=1}^{N} \prod_{i=1}^{p} \frac{1}{1-x_{i} \omega_{a}} \prod_{\alpha=1}^{M} \prod_{\lambda=1}^{q} \frac{1}{1-y_{\lambda} \widetilde{\omega}_{\alpha}} \prod_{b=1}^{N} \prod_{\rho=1}^{q}\left(1+y_{\rho} \omega_{b}\right) \prod_{\beta=1}^{M} \prod_{j=1}^{p}\left(1+x_{j} \widetilde{\omega}_{\beta}\right)
$$

where the first two terms correspond to bosonic excitations of $\phi_{a i}$ and $\widetilde{\phi}_{\alpha \lambda}$ while the others are fermionic contributions of $\psi_{a \lambda}$ and $\widetilde{\psi}_{\alpha i}$.

To project onto the physical states we will carry out a contour integration over the gauge fugacity parameters $\omega_{a}$ and $\widetilde{\omega}_{\alpha}$ in such a way that only gauge invariant states are picked up as a contour integration allows us to compute infinite sums by reducing them to finite sums of residues at poles.

According to the trace parts (4.19) and (4.20) of the Gauss law constraints and the sign factor (6.6), the physical states should carry charge $k$ for each of the Cartan of the $\mathrm{U}(N)$ and charge $k$ for each of the Cartan of the $\mathrm{U}(M)$. Therefore we introduce poles of order $k+1$ and $k+1$ by adding the factors $\prod_{a} \frac{1}{\omega_{a}^{k}}$ and $\prod_{\alpha} \frac{1}{\widetilde{\omega}_{\alpha}^{k}}$ respectively.

As we deal with integration with respect to the elements $\omega_{a}$ and $\widetilde{\omega}_{\alpha}$ of the $\mathrm{U}(N \mid M)$ supermatrix, we will introduce the $\mathrm{U}(N \mid M)$ Berezinian measure [39]. Taking these additional factors into the product of the two contributions (6.11) and (6.12), one can express the partition function as

$$
\begin{aligned}
\mathcal{Z}= & \frac{1}{N !} \frac{1}{M !}\left(\prod_{a=1}^{N} \frac{1}{2 \pi i} \oint_{\Gamma} \frac{d \omega_{a}}{\omega_{a}^{k+1}}\right)\left(\prod_{\alpha=1}^{M} \frac{1}{2 \pi i} \oint_{\widetilde{\Gamma}} \frac{d \widetilde{\omega}_{\alpha}}{\widetilde{\omega}_{\alpha}^{k+1}}\right)\left(\frac{\prod_{b \neq c}^{N}\left(1-\frac{\omega_{b}}{\omega_{c}}\right) \prod_{\beta \neq \gamma}^{M}\left(1-\frac{\widetilde{\omega}_{\beta}}{\widetilde{\omega}_{\gamma}}\right)}{\prod_{d=1}^{N} \prod_{\delta=1}^{M}\left(1+\frac{\widetilde{\omega}_{\delta}}{\omega_{d}}\right)\left(1+\frac{\omega_{d}}{\widetilde{\omega}_{\delta}}\right)}\right) \\
& \times\left(\frac{\prod_{a=1}^{N} \prod_{\alpha=1}^{M}\left(1+q \frac{\widetilde{\omega}_{\alpha}}{\omega_{a}}\right)\left(1+q \frac{\omega_{a}}{\widetilde{\omega}_{\alpha}}\right)}{\prod_{a, b=1}^{N}\left(1-q \frac{\omega_{a}}{\omega_{b}}\right) \prod_{\alpha, \beta=1}^{M}\left(1-q \widetilde{\widetilde{\omega}}_{\widetilde{\omega}_{\beta}}\right)}\right)\left(\frac{\prod_{a=1}^{N} \prod_{\lambda=1}^{q}\left(1+y_{\lambda} \omega_{a}\right) \prod_{\alpha=1}^{M} \prod_{i=1}^{p}\left(1+x_{i} \widetilde{\omega}_{\alpha}\right)}{\prod_{a=1}^{N} \prod_{i=1}^{p}\left(1-x_{i} \omega_{a}\right) \prod_{\alpha=1}^{M} \prod_{\lambda=1}^{q}\left(1-y_{\lambda} \widetilde{\omega}_{\alpha}\right)}\right)
\end{aligned}
$$

where $\Gamma$ and $\widetilde{\Gamma}$ are the $N$-dimensional cycle and $M$-dimensional cycle respectively.

Using the completeness relation [40]

$$
\frac{\prod_{a=1}^{N} \prod_{\lambda=1}^{q}\left(1+y_{\lambda} \omega_{a}\right) \prod_{\alpha=1}^{M} \prod_{i=1}^{p}\left(1+x_{i} \widetilde{\omega}_{\alpha}\right)}{\prod_{a=1}^{N} \prod_{i=1}^{p}\left(1-x_{i} \omega_{a}\right) \prod_{\alpha=1}^{M} \prod_{\lambda=1}^{q}\left(1-y_{\lambda} \widetilde{\omega}_{\alpha}\right)}=\sum_{\lambda} s_{\lambda}(x / y) s_{\lambda}(\omega / \widetilde{\omega})
$$

of the supersymmetric Schur polynomial $s_{\lambda}(x / y)$ and the definition

$$
\begin{aligned}
P_{\lambda}(x ; q) & :=\frac{1}{v_{\lambda}} \sum_{w \in S_{N}} w\left\{x_{1}^{\lambda_{1}} x_{2}^{\lambda_{2}} \cdots x_{N}^{\lambda_{N}} \prod_{i>j} \frac{\left(1-q \frac{x_{i}}{x_{j}}\right)}{\left(1-\frac{x_{i}}{x_{j}}\right)}\right\} \\
& =\sum_{w \in S_{N} \backslash S_{N}^{\lambda}} w\left\{x_{1}^{\lambda_{1}} \cdots x_{N}^{\lambda_{N}} \prod_{\lambda_{i}<\lambda_{j}} \frac{\left(1-q \frac{x_{i}}{x_{j}}\right)}{\left(1-\frac{x_{i}}{x_{j}}\right)}\right\}
\end{aligned}
$$


of the Hall-Littlewood polynomial, where

$$
v_{\lambda}:=\frac{\varphi_{N-l(\lambda)} \prod_{j \geq 1} \varphi_{m_{j}(\lambda)}}{(1-q)^{N}}, \quad \quad \varphi_{m}:=\prod_{i=1}^{m}\left(1-q^{i}\right),
$$

$S_{N}^{\lambda}$ is the set of permutations that fix $\lambda, l(\lambda)$ is the length of the partition $\lambda$, and $m_{j}(\lambda)$ is the multiplicity of the partition $\lambda$, we can write

$$
\begin{aligned}
\mathcal{Z}_{\widehat{\Phi}} & =\sum_{\lambda} s_{\lambda}(x / y) s_{\lambda}(\omega / \widetilde{\omega}), \\
\prod_{a=1}^{N} \frac{1}{\omega_{a}^{k}} & =P_{\left(k^{N}\right)}\left(\omega^{-1} ; q\right), \quad \prod_{\alpha=1}^{M} \frac{1}{\widetilde{\omega}_{\alpha}^{k}}=P_{\left(k^{M}\right)}\left(\widetilde{\omega}^{-1} ; q\right) .
\end{aligned}
$$

Making use of the relations (6.14), (6.18) and (6.19), we can express the partition function (6.13) as

$$
\begin{aligned}
\mathcal{Z}= & \sum_{\lambda} s_{\lambda}(x / y) \frac{1}{N !} \frac{1}{M !}\left(\prod_{a=1}^{N} \frac{1}{2 \pi i} \oint_{\Gamma} \frac{d \omega_{a}}{\omega_{a}}\right)\left(\prod_{\alpha=1}^{M} \frac{1}{2 \pi i} \oint_{\widetilde{\Gamma}} \frac{d \widetilde{\omega}_{\alpha}}{\widetilde{\omega}_{\alpha}}\right) \\
& \times s_{\lambda}(\omega / \widetilde{\omega}) P_{\left(k^{N}\right)}\left(\omega^{-1} ; q\right) P_{\left(k^{M}\right)}\left(\widetilde{\omega}^{-1} ; q\right) \\
& \times\left(\frac{\prod_{b \neq c}^{N}\left(1-\frac{\omega_{b}}{\omega_{c}}\right) \prod_{\beta \neq \gamma}^{M}\left(1-\frac{\widetilde{\omega}_{\beta}}{\widetilde{\omega}_{\gamma}}\right)}{\prod_{d=1}^{N} \prod_{\delta=1}^{M}\left(1+\frac{\widetilde{\omega}_{\delta}}{\omega_{d}}\right)\left(1+\frac{\omega_{d}}{\widetilde{\omega}_{\delta}}\right)}\right)\left(\frac{\prod_{a=1}^{N} \prod_{\alpha=1}^{M}\left(1+q \frac{\widetilde{\omega}_{\alpha}}{\omega_{a}}\right)\left(1+q \widetilde{\omega}_{\tilde{\omega}_{\alpha}}\right)}{\prod_{a, b=1}^{N}\left(1-q \frac{\omega_{a}}{\omega_{b}}\right) \prod_{\alpha, \beta=1}^{M}\left(1-q \frac{\widetilde{\omega}_{\alpha}}{\widetilde{\omega}_{\beta}}\right)}\right)
\end{aligned}
$$

Although we have not precisely yet understood the issue of choice of integration contour, it would be very important as we are now considering the symmetries of Lie superalgebra whose representation and (super)characters have rather rich structures. While the integration contour of simple unit circles would give us partition function contributed from singlet sectors, other non-trivial contour picking up specific poles may realize non-singlet sectors. In the next subsection, we will give an explicit computation for $M=0$ by taking simply unit circles and comment on general cases in subsection 6.3.

\subsection{Computation for $\mathrm{U}(N)$}

Let us consider the case where the gauge symmetry is ordinary $\mathrm{U}(N)$ and the coupling $\omega$ is very large. The contributions (6.11) from the supermatrix field $\widehat{Z}^{\dagger}$ simplify as

$$
\mathcal{Z}_{Z}=\prod_{a, b=1}^{N} \frac{1}{1-q \frac{\omega_{a}}{\omega_{b}}}
$$

and the contributions (6.12) from the supervector field $\widehat{\Phi}$ only contain two parts

$$
\begin{aligned}
& \mathcal{Z}_{\phi}=\prod_{a=1}^{N} \prod_{i=1}^{p} \frac{1}{1-x_{i} \omega_{a}}=\sum_{\lambda} s_{\lambda}(x) s_{\lambda}(\omega), \\
& \mathcal{Z}_{\psi}=\prod_{a=1}^{N} \prod_{\lambda=1}^{q}\left(1+y_{\lambda} \omega_{a}\right)=\sum_{\mu} s_{\mu}(y) s_{\mu^{\prime}}(\omega)
\end{aligned}
$$


where $\mu^{\prime}$ is the conjugate of a partition $\mu$ whose Young diagram is the transpose of that of $\mu$. Thus the integral expression (6.20) reduces to

$$
\begin{aligned}
\mathcal{Z}= & \sum_{\lambda, \mu} s_{\lambda}(x) s_{\mu}(y) \frac{1}{N !} \prod_{a=1}^{N} \frac{1}{2 \pi i} \oint_{C} \frac{d \omega_{a}}{\omega_{a}} \frac{\prod_{b \neq c}^{N}\left(1-\frac{\omega_{b}}{\omega_{c}}\right)}{\prod_{a, b=1}^{N}\left(1-q \frac{\omega_{a}}{\omega_{b}}\right)} s_{\lambda}(\omega) s_{\mu^{\prime}}(\omega) P_{\left(k^{N}\right)}\left(\omega^{-1} ; q\right) \\
= & \sum_{\lambda, \mu, \eta, \rho} s_{\lambda}(x) s_{\mu}(y) \frac{1}{N !} \prod_{a=1}^{N} \frac{1}{2 \pi i} \oint_{C} \frac{d \omega_{a}}{\prod_{a}} \frac{\prod_{b \neq c}^{N}\left(1-\frac{\omega_{b}}{\omega_{c}}\right)}{\prod_{a, b=1}^{N}\left(1-q \frac{\omega_{a}}{\omega_{b}}\right)} \\
& \times \sum_{\eta, \rho} c_{\lambda \mu^{\prime}}^{\eta} K_{\eta, \rho}(q) P_{\rho}(\omega ; q) P_{\left(k^{N}\right)}\left(\omega^{-1} ; q\right) .
\end{aligned}
$$

On the second line we have used the relation

$$
s_{\lambda}(\omega) s_{\mu^{\prime}}(\omega)=\sum_{\eta} c_{\lambda \mu^{\prime}}^{\eta} s_{\eta}(\omega)=\sum_{\eta, \rho} c_{\lambda \mu^{\prime}}^{\eta} K_{\eta, \rho}(q) P_{\rho}(\omega ; q)
$$

where $c_{\mu \nu}^{\lambda}$ are the Littlewood-Richardson coefficients [41] and $K_{\lambda, \mu}(q)$ are the Kostka polynomials which are defined by

$$
s_{\lambda}(x)=\sum_{\mu} K_{\lambda, \mu}(q) P_{\mu}(x ; q) .
$$

Furthermore, using the orthogonal property

$$
\left\langle P_{\lambda}(x ; q), P_{\mu}\left(x^{-1} ; q\right)\right\rangle_{P}=\frac{1}{v_{\lambda}} \delta_{\lambda, \mu}
$$

of the Hall-Littlewood polynomials with respect to the following inner product

$$
\left\langle f_{\lambda}(\omega ; q), g_{\mu}\left(\omega^{-1} ; q\right)\right\rangle_{P}:=\frac{1}{N !} \prod_{a=1}^{N} \frac{1}{2 \pi i} \oint_{C} \frac{d \omega_{a}}{\omega_{a}} \frac{\prod_{a \neq b}\left(1-\frac{\omega_{a}}{\omega_{b}}\right)}{\prod_{a \neq b}\left(1-q \frac{\omega_{a}}{\omega_{b}}\right)} f_{\lambda}(\omega) g_{\mu}\left(\omega^{-1}\right),
$$

we can rewrite the partition function (6.24) as

$$
\mathcal{Z}=\prod_{i=1}^{N} \frac{1}{\left(1-q^{i}\right)} \sum_{\lambda, \mu, \eta} c_{\lambda \mu^{\prime}}^{\eta} K_{\eta, k^{N}}(q) s_{\lambda}(x) s_{\mu}(y) .
$$

According to the relation [40]

$$
s_{\lambda}(x / y)=\sum_{\mu, \nu} c_{\mu \nu}^{\lambda} s_{\mu}(x) s_{\nu^{\prime}}(y)
$$

the expression (6.29) can be expressed as

$$
\mathcal{Z}=\prod_{i=1}^{N} \frac{1}{\left(1-q^{i}\right)} \sum_{\mu} K_{\mu, k^{N}}(q) s_{\mu}(x / y)
$$


where $s_{\mu}(x / y)$ is the supersymmetric Schur polynomial. Here the summation is taken over the partitions $\mu$ which obey

$$
\begin{aligned}
|\mu| & =k N, \\
q \leq l(\mu) & \leq p+q, \\
\mu_{p+1} & \leq q .
\end{aligned}
$$

The first condition (6.33) is required for non-trivial $K_{\mu, k^{N}}(q)$ whereas the other conditions (6.33) and (6.34) are for non-zero valued $s_{\mu}(x / y)$ as the supersymmetric Schur polynomial $s_{\mu}(x / y)$ vanishes when $\mu_{p+1}>q$.

As the modified Hall-Littlewood polynomials $Q_{\mu}^{\prime}(x ; q)$ are defined by

$$
Q_{\mu}^{\prime}(x ; q):=\sum_{\lambda} K_{\lambda, \mu}(q) s_{\lambda}(x)
$$

we will define the supersymmetric modified Hall-Littlewood polynomial $Q_{\mu}^{\prime}(x / y ; q)$ by

$$
Q_{\mu}^{\prime}(x / y ; q):=\sum_{\lambda} K_{\lambda, \mu}(q) s_{\lambda}(x / y)
$$

Then the partition function is expressed as

$$
\mathcal{Z}=\prod_{i=1}^{N} \frac{1}{\left(1-q^{i}\right)} Q_{k^{N}}^{\prime}(x / y ; q) .
$$

Further study of properties of the supersymmetric modified Hall-Littlewood polynomials (6.35) is intriguing. In particular, it would be desirable to understand the large $N$ behavior of the Kostka polynomials as the branching coefficient of $\widehat{\mathfrak{s u}}(p \mid q) / \mathfrak{s u}(p \mid q)$ as in the ordinary case $[42,43]$.

\subsection{Comments on general case}

Although it would be important to study the residues for different choices of contours of the integral (6.20), we will not get into any details of these issues in this paper. Instead, we will comment on some implications of the resulting expression (6.20). To have a welldefined partition function from the integration (6.20), it is expected that the integration can be performed by using the orthogonal property of certain functions with respect to $\omega$ and $\widetilde{\omega}$. Provided that the supersymmetric Schur polynomial $s_{\lambda}(\omega / \widetilde{\omega})$ in $(6.20)$ is expanded in terms of the supersymmetric Hall-Littlewood polynomial $P_{\mu}(x / y ; q),{ }^{6}$

$$
s_{\lambda}(\omega / \widetilde{\omega})=\sum_{\mu} K_{\lambda \mu}(q) P_{\mu}(\omega / \widetilde{\omega} ; q),
$$

the second line in (6.20), equipped with the expressions (6.15) in terms of permutation of variables, would be regarded as the dual of $P_{\mu}(\omega / \widetilde{\omega} ; q)$. In fact, it takes the form of a generalization of the Berele-Regev formula [44]

$$
s_{\lambda}(\omega / \widetilde{\omega})=\prod_{a=1}^{N} \prod_{\alpha=1}^{M}\left(\omega_{a}+\widetilde{\omega}_{\alpha}\right) s_{\lambda}(\omega) s_{\lambda^{\prime}}(\widetilde{\omega}) .
$$

\footnotetext{
${ }^{6}$ This is the supersymmetric generalization of the definition (6.26) of the Kostka polynomial.
} 
It would be also interesting to observe that the supersymmetric Schur polynomial $s_{\lambda}(x / y)$ is alternatively expanded in terms of two Hall-Littlewood polynomials [45, 46]

$$
s_{\lambda}(x / y)=\sum_{\mu, \eta} K_{\lambda, \mu \mid \eta}(q) P_{\mu}(x ; q) P_{\eta}(y ; q),
$$

which defines the Kostka polynomial $K_{\lambda, \mu \mid \eta}(q)$ and that it is expanded in terms of supersymmetric monomial functions $m_{\mu}(x / y)[40]$

$$
s_{\lambda}(x / y)=\sum_{\mu} K_{\lambda \mu} m_{\mu}(x / y)
$$

where $K_{\lambda \mu}$ is the Kostka number. Since the supersymmetric Hall-Littlewood polynomials $P_{\mu}(x / y ; q)$ may interpolate between the supersymmetric Schur polynomials when $q=0$ and the supersymmetric monomial functions when $q=1$, these relations may help us proceed to further survey of the supersymmetric Hall-Littlewood polynomials $P_{\mu}(x / y ; q)$.

In the partition function (6.11) all states constructed from the supermatrix field $\widehat{Z}_{A B}$ have been picked up. However, there are distinguished operators with different structures of the contracted gauge indices: among themselves, with antisymmetric invariant tensor, with the supervector fields. There will exist operators $\left(Z^{\dagger n}\right)_{a b},\left(\widetilde{Z}^{\dagger n}\right)_{\alpha \beta}$ as a product of $Z^{\dagger}$ 's with gauge indices contracted among them so that the antifundamental index of each operator is contracted with the fundamental index of the following operator. If we start with a set of states with minimal basis constructed by the operator $\left\{Z_{a b}^{\dagger}\right\}$ and next count a set of states with $\left\{Z_{a b}^{\dagger}\right\}$ being replaced with $\left\{\left(Z^{\dagger n}\right)_{a b}\right\}$ as they have the same gauge charges but $n$ units of the energy of $\left\{Z_{a b}^{\dagger}\right\}$, then the corresponding partition function may take the form of

$$
\mathcal{Z}_{\widehat{Z}}=\prod \frac{1}{1-q^{n} \frac{\omega_{a}}{\omega_{b}}} \frac{1}{1-q^{n} \frac{\widetilde{\omega}_{\alpha}}{\widetilde{\omega}_{\beta}}}\left(1+q^{n} \frac{\widetilde{\omega}_{\alpha}}{\omega_{b}}\right)\left(1+q^{n} \frac{\omega_{a}}{\widetilde{\omega}_{\beta}}\right)
$$

by taking some appropriate constrained product to avoid over counting. This has the same form as the affine Weyl denominator $\widehat{R}$ (divided by Weyl denominator $R$ ) [47]

$$
\widehat{R}:=R \prod_{n=1}^{\infty}\left[\left(1-q^{n}\right)^{l} \frac{\prod_{\alpha \in \Delta_{0}}\left(1-q^{n} e^{\alpha}\right)}{\prod_{\alpha \in \Delta_{1}}\left(1+q^{n} e^{\alpha}\right)}\right]
$$

where $R$ is the Weyl denominator defined by [47]

$$
R:=\frac{\prod_{\alpha \in \Delta_{0}}\left(1-e^{\alpha}\right)}{\prod_{\alpha \in \Delta_{1}}\left(1+e^{\alpha}\right)}
$$

and $l$ is the quantum number of the Virasoro generator $L_{0}$, which is equal to the rank for $N \neq M$, under the identifications $\omega_{a}:=e^{-\epsilon_{a}}$ and $\widetilde{\omega}_{\alpha}:=e^{-\delta_{\alpha}}$ where $\epsilon_{a}, a=1, \cdots, N$ and $\delta_{\alpha}, \alpha=1, \cdots, M$ is a basis of the root space (see (3.56) and (3.57)). We also note that the factor of the Berezinian measure

$$
\frac{\prod_{b \neq c}^{N}\left(1-\frac{\omega_{b}}{\omega_{c}}\right) \prod_{\beta \neq \gamma}^{M}\left(1-\frac{\widetilde{\omega}_{\beta}}{\widetilde{\omega}_{\gamma}}\right)}{\prod_{d=1}^{N} \prod_{\delta=1}^{M}\left(1+\frac{\widetilde{\omega}_{\delta}}{\omega_{d}}\right)\left(1+\frac{\omega_{d}}{\widetilde{\omega}_{\delta}}\right)}
$$


in (6.13) has a close similarity with the Weyl denominator. Since the affine Weyl denominator is associated to Ramanujan's mock theta function, as pointed out by Kac and Wakimoto [47, 48] (also see [49]), the partition function (6.42) would indicate the property of mock modularity.

\section{Discussion}

We have studied a $(0+1)$-dimensional $\mathrm{U}(N \mid M)$ matrix Chern-Simons quantum mechanics with an $\mathrm{SU}(p \mid q)$ global symmetry. We have proposed it as a description of a system consisting of $N$ vortices and $M$ antivortices with $\mathrm{SU}(p \mid q)$ spin degrees of freedom. At the classical level, we have seen that the model can be viewed as a generalized Calogero model with $\mathrm{SU}(p \mid q)$ spin degrees of freedom. We have also found two types of classical ground states which admit non-trivial configuration of fermionic matrix fields. They are similar to the two types of vortex-antivortex pairs; parallel polarized vortex-antivortex pairs with negative energy and antiparallel polarized vortex-antivortex pairs with positive energy. Meanwhile we have provided a general expression of the partition function in an integral form and we have found that the expression can be explicitly written in terms of Kostka polynomials and super-Schur polynomials as a generalization of [10].

It is physically important to obtain further understanding of vortex-antivortex systems from the $\mathrm{U}(N \mid M)$ matrix Chern-Simons models. In particular, it is intriguing to find new explanations and predictions in quantum Hall physics beyond the well-known features of the Laughlin theory. For instance, as in the ordinary matrix Chern-Simons models $[1,2]$, we would like to understand the level quantization and its relation to the filling fractions of the quantum Hall states. Besides, it would be interesting to construct and understand generalized wavefunctions valid for the superdeterminant states which we found in this work.

Further understanding of the mathematical structure would be intriguing. Although we have found that the current operators constructed from matrix degrees of freedom give rise to the affine Lie superalgebra in the large $N$ limit, we would like to support our results with a rigorous treatment of the partition function. In addition, for general supergroup we have not found an explicit expression in terms of polynomials. This is due to the lack of knowledge of the orthogonal properties and we expect that it could be achieved by defining supersymmetric Hall-Littlewood polynomials. But we leave this problem for future study.

In addition, it is an open question even for the ordinary Lie algebra to understand the underlying larger algebra without taking a large $N$ limit. Interestingly it has been argued $[50,51]$ that in the related Polychronakos spin chain model [52] the Yangian symmetry can be embedded in the WZW model. Specifically, the partition function becomes the character for the WZW model at level one in the large $N$ limit, as the first Yangian invariant operator is identified with the Virasoro generator.

Another attractive future direction is to explore the gravitational dual of the $(0+1)$ dimensional matrix Chern-Simons quantum mechanics as it may be useful to understand the holographic dual description of generally conjectured infinite dimensional symmetry in 
two-dimensional gravity. Geroch showed [53] that a hidden symmetry in two-dimensional gravity is infinite dimensional, known as the Geroch group, which indicates that Einstein gravity is integrable after reducing to two-dimensions. Julia demonstrated $[54,55]$ that the Geroch group is the affine Lie algebra $A_{1}^{(1)}$. Since Dorey, Tong and Turner's recent work [10] and our result show that the quantum mechanical systems with $N$ degrees of freedom realize the affine Kac-Moody symmetry in the large $N$ limit, it may help us to understand the underlying infinite-dimensional symmetry structure in two-dimensional gravity and further lifted symmetry in higher dimensional gravity. There has also been recent work on matrix $\mathrm{U}(N)$ Chern-Simons quantum mechanics systems with $N_{f}$ fundamental and antifundamental fields [56]. These models, also related to Calogero systems, describe FZZT branes in Liouville theory and also two-dimensional blackholes. It was also shown [56] that these models exhibit a phase transition at large $N$ and $N_{f}$, and an intriguing relation of the grand-canonical partition function to the Toda intergrable hierarchy was found. It will be interesting to explore these issues for our supergroup models.

Further possible applications of the matrix Chern-Simons model could be found in string and M-theory. In the type IIB string theory the D1-branes which end on the intersecting D3-branes are vortices in the effective $3 \mathrm{~d}$ gauge theory, and the relation between the vortex D1-branes and the matrix Chern-Simons model has been examined in [12]. In [57] intersecting D3-branes and NS5-branes in curved spacetime are shown to correspond to supergroup Chern-Simons theory. It would be interesting to explore the relation between further attached vortex-like D1-branes involving the supergroup symmetry and our supergroup Chern-Simons matrix model. In M-theory intersecting M2-branes can be viewed as vortices in the Chern-Simons matter theory. In this brane setup the large $N$ limit of the Chern-Simons matrix model corresponds to an infinite number of intersecting M2-branes, which would lead to an M5-brane as a condensate of M2-branes. In [58, 59] we found that a certain configuration of intersecting M2-M5 branes on a two-dimensional plane can be effectively described by the supergroup WZW model associated to the affine Lie superalgebra. Since we have found a connection to the affine Lie superalgebra in this work, we believe that further physical explanation and application can be available in string and M-theory.

\section{Acknowledgments}

We would like to thank Heng-Yu Chen, Chong-Sun Chu, Nick Dorey, Amihay Hanany, Taro Kimura, Anatoli Kirillov, Alexios Polychronakos, David Tong and Carl Turner for useful discussions and comments. TO thanks the organizers of the Topological Field Theories, String theory and Matrix Models in Moscow for hospitality during the course of the work. TO is supported by MOST under the Grant No.106-2811-M-002-053. DJS thanks the Taiwan NCTS Physics Division for support and hospitality to enable a productive visit to NTHU and also NTU where some of this work was carried out. DJS is supported in part by the STFC Consolidated Grant ST/P000371/1. 


\section{A More classical ground states}

Consider the case with $\alpha_{N N}=\widetilde{\alpha}_{M M}=0$ as the solution to (3.47) and take

$$
\beta_{a}=(N-a) \omega, \quad \widetilde{\beta}_{\alpha}=(M-\alpha) \omega .
$$

As $\alpha_{N N}=\widetilde{\alpha}_{M M}$, this would imply the occurrence of enhanced symmetry. The configurations (A.1) tell us that the fields $Z_{a b}, \widetilde{Z}_{\alpha \beta}, A_{a \alpha}$ and $B_{\alpha a}$ have general forms

$$
\begin{aligned}
& Z=\left(\begin{array}{ccccc}
0 & Z_{12} & & & \\
& 0 & Z_{23} & & \\
& \ddots & \ddots & \\
& & 0 & Z_{N-1 N} \\
& & & 0
\end{array}\right), \quad \widetilde{Z}=\left(\begin{array}{cccc}
0 & \widetilde{Z}_{12} & & \\
& \ddots & \ddots & \\
& & 0 & \widetilde{Z}_{M-1 M} \\
& & & 0
\end{array}\right),
\end{aligned}
$$

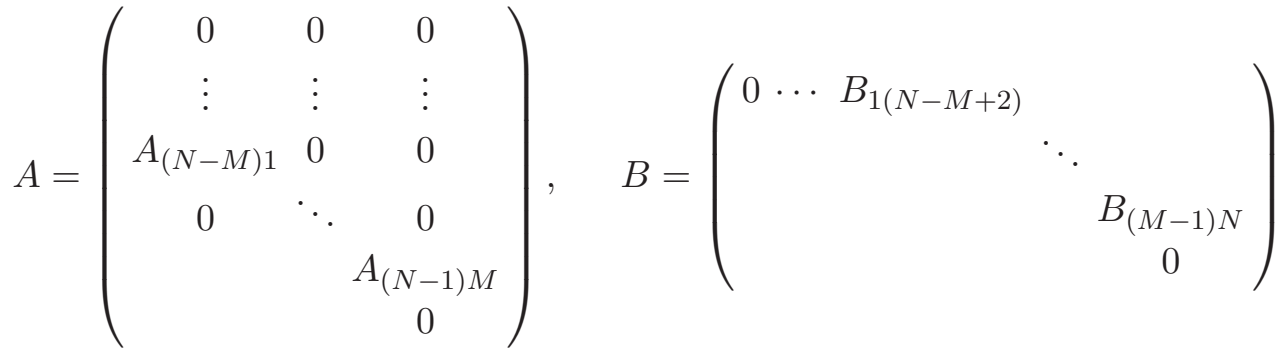

where the elements $Z_{a(a+1)}, \widetilde{Z}_{\alpha(\alpha+1)}, A_{a(M-N+1+a)}, B_{\alpha(N-M+1+\alpha)}$ are the only components allowed to have non-zero values. Then the Gauss law constraints (3.10)-(3.13) become

$$
\begin{aligned}
& \left(\left|Z_{a(a+1)}\right|^{2}-\left|Z_{a(a-1)}\right|^{2}+A_{a(a-N+M+1)} A_{(a-N+M+1) b}^{\dagger}-B_{a(a-N+M-1)}^{\dagger} B_{(a-N+M-1) b}\right) \delta_{a b} \\
& \quad+\left(\kappa(N-M)+|z|^{2}\right) \delta_{a N} \delta_{b N}=\kappa \delta_{a b}, \\
& \left(\left|\widetilde{Z}_{\alpha(\alpha+1)}\right|^{2}-\left|\widetilde{Z}_{\alpha(\alpha-1)}\right|^{2}+B_{\alpha(\alpha+N-M+1)} B_{(a+N-M+1) b}^{\dagger}-A_{\alpha(\alpha+N-M-1)}^{\dagger} A_{(\alpha+N-M-1) \beta}\right) \delta_{\alpha \beta} \\
& \quad+|z|^{2} \delta_{\alpha M} \delta_{\beta M}=\kappa \delta_{\alpha \beta}, \\
& B_{\alpha(\alpha+N-M+1)} Z_{(\alpha+N-M+1) \alpha+N-M}^{\dagger}-\widetilde{Z}_{\alpha(\alpha-1)}^{\dagger} B_{(\alpha-1)(\alpha+N-M)} \\
& \quad-A_{\alpha(\alpha+N-M-1)}^{\dagger} Z_{(\alpha+N-M-1)(\alpha+N-M)}+\widetilde{Z}_{\alpha(\alpha+1)} A_{(\alpha+1)(\alpha+N-M)}^{\dagger}+z y^{\dagger} \delta_{\alpha M}=0 .
\end{aligned}
$$

We define the following quantities:

$$
\begin{aligned}
z_{a} & =\frac{1}{\kappa}\left|Z_{a(a+1)}\right|^{2}, \quad a \in\{1,2, \ldots, N-1\}, \\
\widetilde{z}_{\alpha} & =\frac{1}{\kappa}\left|\widetilde{Z}_{\alpha(\alpha+1)}\right|^{2}, \quad \alpha \in\{1,2, \ldots, M-1\}, \\
\mathcal{A}_{a} & =\frac{1}{\kappa} A_{a(a-N+M+1)} A_{(a-N+M+1) a}^{\dagger}, \quad a \in\{N-M, N-M+1, \ldots, N-1\}, \\
\mathcal{B}_{a} & =\frac{1}{\kappa} B_{a(a-N+M-1)}^{\dagger} B_{(a-N+M-1) a}, \quad a \in\{N-M+2, N-M+3, \ldots, N\} .
\end{aligned}
$$

Above we have assumed $N>M$. In the case $N=M$ note that there is one fewer $\mathcal{A}_{a}$ as clearly the value $a=0$ is not allowed as in (A.2) there is no component $A_{(N-M) 1}=A_{01}$. 
Then the Gauss law constraints become, assuming $N \geq M+2$

$$
\begin{aligned}
\tilde{z}_{\alpha} & =\alpha-\sum_{\gamma=N-M}^{\alpha+N-M-1} \mathcal{A}_{\gamma}+\sum_{\gamma=N-M+2}^{\alpha+N-M+1} \mathcal{B}_{\gamma}, \alpha \in\{1,2, \ldots, M-1\} \\
\frac{1}{\kappa}|z|^{2} & =M-\sum_{\gamma=N-M}^{N-1} \mathcal{A}_{\gamma}+\sum_{\gamma=N-M+2}^{N} \mathcal{B}_{\gamma} \\
z_{a} & =a, \quad a \in\{1,2, \ldots, N-M-1\} \\
z_{N-M} & =N-M-\mathcal{A}_{N-M} \\
z_{N-M+1} & =N-M+1-\mathcal{A}_{N-M}-\mathcal{A}_{N-M+1} \\
z_{a} & =a-\sum_{\gamma=N-M}^{a} \mathcal{A}_{\gamma}+\sum_{\gamma=N-M+2}^{a} \mathcal{B}_{\gamma}, a \in\{N-M+2, N-M+3, \ldots, N-1\}
\end{aligned}
$$

$$
|x|^{2}-y^{\dagger} y-|z|^{2}=(N-M) \kappa
$$

along with (A.5).

In the case $N=M+1$ we don't have equation (A.9) while in the case $N=M$ the Gauss law constraints are instead

$$
\begin{aligned}
\widetilde{z}_{1} & =1+\mathcal{B}_{2}, \\
\widetilde{z}_{\alpha} & =\alpha-\sum_{\gamma=1}^{\alpha-1} \mathcal{A}_{\gamma}+\sum_{\gamma=2}^{\alpha+1} \mathcal{B}_{\gamma}, \quad \alpha \in\{2, \ldots, N-1\}, \\
\frac{1}{\kappa}|z|^{2} & =N-\sum_{\gamma=1}^{N-1} \mathcal{A}_{\gamma}+\sum_{\gamma=2}^{N} \mathcal{B}_{\gamma}, \\
z_{1} & =1-\mathcal{A}_{1}, \\
z_{a} & =a-\sum_{\gamma=1}^{a} \mathcal{A}_{\gamma}+\sum_{\gamma=2}^{a} \mathcal{B}_{\gamma}, \quad a \in\{2,3, \ldots, N-1\}, \\
|x|^{2}-y^{\dagger} y-|z|^{2} & =0
\end{aligned}
$$

along with (A.5).

Open Access. This article is distributed under the terms of the Creative Commons Attribution License (CC-BY 4.0), which permits any use, distribution and reproduction in any medium, provided the original author(s) and source are credited.

\section{References}

[1] A.P. Polychronakos, Quantum Hall states as matrix Chern-Simons theory, JHEP 04 (2001) 011 [hep-th/0103013] [INSPIRE].

[2] N. Dorey, D. Tong and C. Turner, Matrix model for non-Abelian quantum Hall states, Phys. Rev. B 94 (2016) 085114 [arXiv: 1603.09688] [INSPIRE]. 
[3] L. Susskind, The Quantum Hall fluid and noncommutative Chern-Simons theory, hep-th/0101029 [INSPIRE].

[4] R.B. Laughlin, Anomalous quantum Hall effect: An Incompressible quantum fluid with fractionallycharged excitations, Phys. Rev. Lett. 50 (1983) 1395 [INSPIRE].

[5] S. Hellerman and M. Van Raamsdonk, Quantum Hall physics equals noncommutative field theory, JHEP 10 (2001) 039 [hep-th/0103179] [INSPIRE].

[6] D. Karabali and B. Sakita, Chern-Simons matrix model: Coherent states and relation to Laughlin wavefunctions, Phys. Rev. B 64 (2001) 245316 [hep-th/0106016] [INSPIRE].

[7] D. Karabali and B. Sakita, Orthogonal basis for the energy eigenfunctions of the Chern-Simons matrix model, Phys. Rev. B 65 (2002) 075304 [hep-th/0107168] [INSPIRE].

[8] A. Cappelli and M. Riccardi, Matrix model description of Laughlin Hall states, J. Stat. Mech. 0505 (2005) P05001 [hep-th/0410151] [INSPIRE].

[9] I.D. Rodriguez, Edge excitations of the Chern Simons matrix theory for the FQHE, JHEP 07 (2009) 100 [arXiv:0812.4531] [INSPIRE].

[10] N. Dorey, D. Tong and C. Turner, A Matrix Model for WZW, JHEP 08 (2016) 007 [arXiv: 1604.05711] [INSPIRE].

[11] D. Tong and C. Turner, Quantum Hall effect in supersymmetric Chern-Simons theories, Phys. Rev. B 92 (2015) 235125 [arXiv:1508.00580] [InSPIRE].

[12] D. Tong, A Quantum Hall fluid of vortices, JHEP 02 (2004) 046 [hep-th/0306266] [INSPIRE].

[13] A. Hanany and D. Tong, Vortices, instantons and branes, JHEP 07 (2003) 037 [hep-th/0306150] [INSPIRE].

[14] N.S. Manton, First order vortex dynamics, Annals Phys. 256 (1997) 114 [hep-th/9701027] [INSPIRE].

[15] N.M. Romao, Quantum Chern-Simons vortices on a sphere, J. Math. Phys. 42 (2001) 3445 [hep-th/0010277] [INSPIRE].

[16] N.M. Romao and J.M. Speight, Slow Schroedinger dynamics of gauged vortices, Nonlinearity 17 (2004) 1337 [hep-th/0403215] [INSPIRE].

[17] N.S. Manton, A Remark on the Scattering of BPS Monopoles, Phys. Lett. 110B (1982) 54 [INSPIRE].

[18] J.M. Kosterlitz and D.J. Thouless, Ordering, metastability and phase transitions in two-dimensional systems, J. Phys. C 6 (1973) 1181 [INSPIRE].

[19] M. Oshikawa, Y. B. Kim, K. Shtengel, C. Nayak, and S. Tewari, Topological degeneracy of non-abelian states for dummies, Annals Phys. 322 (2007) 1477.

[20] N. Papanicolaou and P. Spathis, Semitopological solitons in planar ferromagnets, Nonlinearity 12 (1999) 285.

[21] C.F. Barenghi and N.G. Parker, A Primer on Quantum Fluids, in SpringerBriefs in Physics, first edition, Springer, New York U.S.A. (2016).

[22] S. Komineas, Rotating vortex dipoles in ferromagnets, Phys. Rev. Lett. 99 (2007) 117202 [arXiv: 0705.2977]. 
[23] S. Komineas and N. Papanicolaou, Dynamics of vortex-antivortex pairs in ferromagnets, arXiv:0712.3684.

[24] R. Hertel and C.M. Schneider, Exchange explosions: Magnetization dynamics during vortex-antivortex annihilation, Phys. Rev. Lett. 97 (2006) 177202.

[25] K.-S. Lee, S. Choi and S.-K. Kim, Radiation of spin waves from magnetic vortex cores by their dynamic motion and annihilation processes, Appl. Phys. Lett. 87 (2005) 192502

[26] B. Van Waeyenberge et al., Magnetic vortex core reversal by excitation with short bursts of an alternating field, Nature 444 (2006) 461

[27] R. Hertel, S. Gliga, M. Fähnle and C. Schneider, Ultrafast nanomagnetic toggle switching of vortex cores, Phys. Rev. Lett. 98 (2007) 117201

[28] O.A. Tretiakov and O. Tchernyshyov, Comment on "Exchange Explosions: Magnetization Dynamics during Vortex-Antivortex Annihilation", Phys. Rev. B 75 (2007) 012408 [cond-mat/0611392] [INSPIRE].

[29] J.P. Eisenstein, G.S. Boebinger, L.N. Pfeiffer, K.W. West and S. He, New fractional quantum Hall state in double-layer two-dimensional electron systems, Phys. Rev. Lett. 68 (1992) 1383 [INSPIRE].

[30] Y. Suen, H. Manoharan, X. Ying, M. Santos and M. Shayegan, Origin of the $\nu=1 / 2$ fractional quantum hall state in wide single quantum wells, Phys. Rev. Lett. 72 (1994) 3405.

[31] S. Murphy, J. Eisenstein, G. Boebinger, L. Pfeiffer and K. West, Many-body integer quantum hall effect: evidence for new phase transitions, Phys. Rev. Lett. 72 (1994) 728.

[32] S. Chae et al., Direct observation of the proliferation of ferroelectric loop domains and vortex-antivortex pairs, Phys. Rev. Lett. 108 (2012) 167603 [arXiv:1203.5371].

[33] A. Hierro-Rodriguez et al., Deterministic propagation of vortex-antivortex pairs in magnetic trilayers, Appl. Phys. Lett. 110 (2017) 262402.

[34] L. Brink, A. Turbiner and N. Wyllard, Hidden algebras of the (super)Calogero and Sutherland models, J. Math. Phys. 39 (1998) 1285 [hep-th/9705219] [INSPIRE].

[35] B. Basu-Mallick, H. Ujino and M. Wadati, Exact spectrum and partition function of SU $(m-n)$ supersymmetric Polychronakos model, J. Phys. Soc. Jap. 68 (1999) 3219 [hep-th/9904167] [INSPIRE].

[36] K. Hikami and B. Basu-Mallick, Supersymmetric Polychronakos Spin Chain: Motif, Distribution Function and Character, Nucl. Phys. B 566 (2000) 511 [math-ph/9904033] [INSPIRE].

[37] B. Basu-Mallick and N. Bondyopadhaya, Spectral properties of supersymmetric Polychronakos spin chain associated with $A_{N-1}$ root system, Phys. Lett. A 373 (2009) 2831 [arXiv: 0811.3110] [INSPIRE].

[38] V.G. Kac, Lie Superalgebras, Adv. Math. 26 (1977) 8 [InSPIRE].

[39] F.A. Berezin, Mathematical Physics and Applied Mathematics. Vol. 9: Introduction to superanalysis, D. Reidel Publishing Co., Dordrecht The Netherlands (1987).

[40] E. Moens, Supersymmetric Schur functions and Lie superalgebra representations, Ph.D. Thesis, Ghent University, Ghent Belgium (2007).

[41] I.G. Macdonald, Symmetric functions and Hall polynomials, Oxford Mathematical Monographs, second edition, Oxford University Press, New York U.S.A. (1995). 
[42] A.N. Kirillov, Dilogarithm identities, Prog. Theor. Phys. Suppl. 118 (1995) 61 [hep-th/9408113] [INSPIRE].

[43] A. Nakayashiki and Y. Yamada, Kostka polynomials and energy functions in solvable lattice models, q-alg/9512027 [INSPIRE].

[44] A. Berele and A. Regev, Hook Young-Diagrams With Applications To Combinatorics And To Representations Of Lie-Superalgebras, Adv. Math. 64 (1987) 118 [INSPIRE].

[45] A.N. Kirillov and N.Y. Reshetikhin, The Bethe ansatz and the combinatorics of Young tableaux, Zap. Nauchn. Sem. LOMI 155 (1986) 65 [J. Sov. Math. 41 (1988) 925].

[46] A.N. Kirillov, On the Kostka-Green-Foulkes polynomials and Clebsch-Gordan numbers, J. Geom. Phys. 5 (1988) 365.

[47] V.G. Kac and M. Wakimoto, Integrable highest weight modules over affine superalgebras and number theory, hep-th/9407057 [INSPIRE].

[48] V.G. Kac and M. Wakimoto, Integrable highest weight modules over affine superalgebras and Appell's function, Commun. Math. Phys. 215 (2001) 631.

[49] D. Hickerson, A proof of the mock theta conjectures, Invent. Math. 94 (1988) 639.

[50] K. Hikami, Yangian symmetry and Virasoro character in a lattice spin system with long-range interactions, Nucl. Phys. B 441 (1995) 530 [INSPIRE].

[51] K. Hikami and B. Basu-Mallick, Supersymmetric Polychronakos Spin Chain: Motif, Distribution Function and Character, Nucl. Phys. B 566 (2000) 511 [math-ph/9904033] [INSPIRE].

[52] A.P. Polychronakos, Exact spectrum of $\mathrm{SU}(N)$ spin chain with inverse square exchange, Nucl. Phys. B 419 (1994) 553 [hep-th/9310095] [InSPIRE].

[53] R.P. Geroch, A Method for generating new solutions of Einstein's equation. 2, J. Math. Phys. 13 (1972) 394 [INSPIRE].

[54] S.W. Hawking and M. Rocek eds., Superspace and Supergravity, Proceedings of Nuffield Workshop, Cambridge U.K. (1980).

[55] B. Julia, Infinite Lie Algebras in Physics, in Proceedings of Unified field theories and beyond, Baltimore U.S.A. (1981).

[56] P. Betzios and O. Papadoulaki, FZZT branes and non-singlets of Matrix Quantum Mechanics, arXiv:1711.04369 [INSPIRE].

[57] V. Mikhaylov and E. Witten, Branes And Supergroups, Commun. Math. Phys. 340 (2015) 699 [arXiv:1410.1175] [INSPIRE].

[58] T. Okazaki and D.J. Smith, Topological M-strings and supergroup Wess-Zumino-Witten models, Phys. Rev. D 94 (2016) 065016 [arXiv:1512.06646] [INSPIRE].

[59] T. Okazaki and D.J. Smith, Mock modular index of M2-M5 brane systems, Phys. Rev. D 96 (2017) 026017 [arXiv:1612.07565] [INSPIRE]. 